\title{
Travelling Waves for Adaptive Grid Discretizations of Reaction Diffusion Systems II: Linear Theory
}

\author{
H. J. Hupkes ${ }^{1}$ (D) E. S. Van Vleck ${ }^{2}$
}

Received: 17 October 2019 / Revised: 29 October 2020 / Accepted: 4 January 2021 /

Published online: 6 March 2021

(c) The Author(s) 2021

\begin{abstract}
In this paper we consider an adaptive spatial discretization scheme for the Nagumo PDE. The scheme is a commonly used spatial mesh adaptation method based on equidistributing the arclength of the solution under consideration. We assume that this equidistribution is strictly enforced, which leads to the non-local problem with infinite range interactions that we derived in Hupkes and Van Vleck (J Dyn Differ Equ 28:955, 2016). For small spatial grid-sizes, we establish some useful Fredholm properties for the operator that arises after linearizing our system around the travelling wave solutions to the original Nagumo PDE. In particular, we perform a singular perturbation argument to lift these properties from the natural limiting operator. This limiting operator is a spatially stretched and twisted version of the standard second order differential operator that is associated to the PDE waves.
\end{abstract}

Keyword Travelling waves $\cdot$ Adaptive grids $\cdot$ Singular perturbations $\cdot$ Spatial discretizations Mathematics Subject Classification 2010 Subject Classification 34K31 · 37L15

\section{Introduction}

In this paper we continue the program initiated in [4] to construct travelling wave solutions to adaptive discretization schemes for scalar bistable systems such as the Nagumo PDE

$$
u_{t}=u_{x x}+g_{\mathrm{cub}}(u ; a),
$$

with the cubic nonlinearity

$$
g_{\mathrm{cub}}(u)=u(1-u)(u-a), \quad 0<a<1 .
$$

$凶 \quad$ H. J. Hupkes

hhupkes@math.leidenuniv.nl

E. S. Van Vleck

erikvv@ku.edu

1 Mathematisch Instituut, Universiteit Leiden, P.O. Box 9512, 2300, RA Leiden, The Netherlands

2 Department of Mathematics, University of Kansas, 1460 Jayhawk Blvd, Lawrence, KS 66045, USA 
In particular, we study schemes that aim to equidistribute the arclength of a solution profile equally between gridpoints in order to improve the resolution inside the regions of interest. The main goal here is to understand the linear operators that underpin the dynamics by transferring Fredholm properties from the continuous to the discrete regime.

Sturm-Liouville theory Substituting the travelling wave Ansatz $u(x, t)=\Phi(x+c t)$ into (1.1), we obtain the travelling wave ODE

$$
c \Phi^{\prime}=\Phi^{\prime \prime}+g_{\mathrm{cub}}(\Phi ; a) .
$$

Using a now standard phase-plane analysis [2], one readily shows that (1.3) coupled with the boundary conditions

$$
\Phi(-\infty)=0, \quad \Phi(+\infty)=1
$$

admits a unique solution pair $(\Phi, c)=\left(\Phi_{*}, c_{*}\right)=\left(\Phi_{*}(a), c_{*}(a)\right)$, with

$$
\operatorname{sign}\left(c_{*}(a)\right)=\operatorname{sign}\left(\frac{1}{2}-a\right), \quad \Phi_{*}^{\prime}>0 .
$$

The latter strict monotonicity result is especially useful when using classical Sturm-Liouville theory to study the linear operator

$$
\left[\mathcal{L}_{\mathrm{tw}} v\right](\xi)=-c_{*} v^{\prime}(\xi)+v^{\prime \prime}(\xi)+g_{\mathrm{cub}}^{\prime}\left(\Phi_{*}(\xi) ; a\right) v(\xi)
$$

associated to the linearization of $(1.3)$ around $\left(\Phi_{*}, c_{*}\right)$. Indeed, this theory immediately implies that the spectrum of $\mathcal{L}_{\mathrm{tw}}: H^{2} \rightarrow L^{2}$ lies strictly to the left of the imaginary axis, with the exception of a simple eigenvalue at zero [9].

This result can subsequently be leveraged to conclude that the waves $\left(\Phi_{*}, c_{*}\right)$ are nonlinearly stable [10] and depend smoothly on the parameter $a$. In addition, it can be used to show that the corresponding planar waves $u(x, y, t)=\Phi_{*}\left(x+c_{*} t\right)$ are nonlinearly stable [8] for the two-dimensional Nagumo PDE

$$
u_{t}=u_{x x}+u_{y y}+g_{\mathrm{cub}}(u ; a)
$$

and can be 'bended' to form travelling corners [3]. All these results do not use the comparison principle, allowing the techniques to be readily generalized to multi-component reactiondiffusion equations.

Uniform spatial discretizations We recall the lattice differential equation (LDE)

$$
\dot{U}_{j}(t)=\frac{1}{h^{2}}\left[U_{j-1}(t)+U_{j+1}(t)-2 U_{j}(t)\right]+g_{\text {cub }}\left(U_{j}(t) ; a\right)
$$

that arises by applying a standard nearest-neighbour discretization to the second derivative in (1.1). Travelling wave solutions $U_{j}(t)=\Phi(j h+c t)$ must now satisfy the system

$$
c \Phi^{\prime}(\xi)=\frac{1}{h^{2}}[\Phi(\xi-h)+\Phi(\xi+h)-2 \Phi(\xi)]+g_{\text {cub }}(\Phi(\xi) ; a) .
$$

In the continuum regime $0<h \ll 1$, a natural first step is to construct spatially-discrete waves as small perturbations from the PDE waves $\left(\Phi_{*}, c_{*}\right)$. However, a short inspection shows that the transition between (1.3) and (1.9) is highly singular. Nevertheless, Johann [7] developed a version of the implicit function theorem that can achieve this in some settings. Our inspiration for the present paper however comes from the spectral convergence approach developed by Bates and his coauthors in [1]. 
A key role in this approach is reserved for the linear operator

$$
\left[\mathcal{L}_{h ; \text { unif }} v\right](\xi)=-c v^{\prime}(\xi)+\frac{1}{h^{2}}[v(\xi+h)+v(\xi-h)-2 v(\xi)]+g_{\text {cub }}^{\prime}\left(\Phi_{*}(\xi) ; a\right) v(\xi),
$$

which can be seen as the linearization of (1.9) around the PDE wave $\Phi_{*}$. In fact, it is a singularly perturbed version of the linear operator $\mathcal{L}_{\mathrm{tw}}$ introduced in (1.6). The main contribution in [1] is that Fredholm properties of $\mathcal{L}_{\text {tw }}$ are transferred to $\mathcal{L}_{h \text {; unif }}$. The latter operator can then be used in a standard fashion to close a fixed-point argument and construct a solution to (1.9) that is close to $\left(\Phi_{*}, c_{*}\right)$.

Stated more precisely, the authors fix a constant $\delta>0$ and use the invertibility of $\mathcal{L}_{\text {tw }}+\delta$ to show that also $\mathcal{L}_{h \text {; unif }}+\delta$ is invertible for small $h>0$. In particular, they consider bounded weakly-converging sequences $\left\{v_{j}\right\} \subset H^{1}$ and $\left\{w_{j}\right\} \subset L^{2}$ with $\left(\mathcal{L}_{h \text {; unif }}+\delta\right) v_{j}=w_{j}$ and set out to find a lower bound for $w_{j}$ that is uniform in $\delta$ and $h$. This can be achieved by picking a large compact interval $K$ and extracting a subsequence of $\left\{v_{j}\right\}$ that converges strongly in $L^{2}(K)$. Special care must therefore be taken to rule out the limitless transfer of energy into oscillatory or tail modes, which are not visible in this strong limit. Spectral properties of the (discrete) Laplacian together with the bistable structure of the nonlinearity $g$ provide the control on $\left\{v_{j}\right\}$ that is necessary for this.

The results in [1] are actually strong enough to handle discretizations of the Laplacian that have infinite range interactions. In addition, this approach was recently generalized [11] for use in multi-component reaction-diffusion problems such as the FitzHugh-Nagumo system. We emphasize that this generalization also allows one to establish the stability of the constructed waves, which is an important reason for us to pursue this line of thought in the present paper.

Uniform spatial-temporal discretizations Applying the backward Euler discretization to the remaining derivative in (1.9), we see that fully discretized front solutions

$$
U_{j}(n \Delta t)=\Phi(j+n c \Delta t), \quad \Phi(-\infty)=0, \quad \Phi(+\infty)=1
$$

to the coupled map lattice

$$
\begin{aligned}
\frac{1}{\Delta t}\left[U_{j}(n \Delta t)-U_{j}((n-1) \Delta t)\right]= & \frac{1}{h^{2}}\left[U_{j-1}(n \Delta t)+U_{j+1}(n \Delta t)-2 U_{j}(n \Delta t)\right] \\
& +g_{\text {cub }}\left(U_{j}(n \Delta t) ; a\right) .
\end{aligned}
$$

must satisfy the difference equation

$$
\frac{1}{\Delta t}[\Phi(\xi)-\Phi(\xi-c \Delta t)]=\frac{1}{h^{2}}[\Phi(\xi-h)+\Phi(\xi+h)-2 \Phi(\xi)]+g_{\text {cub }}(\Phi(\xi) ; a) .
$$

Inspired by the approach above, one can set out to understand the fully discrete operator

$$
\begin{aligned}
{\left[\mathcal{L}_{h, \Delta t} v\right](\xi)=} & -\frac{1}{\Delta t}[v(\xi)-v(\xi-c \Delta t)]+\frac{1}{h^{2}}[v(\xi-1)+v(\xi+1)-2 v(\xi)] \\
& +g_{\text {cub }}^{\prime}(\Phi(\xi) ; a) v(\xi),
\end{aligned}
$$

in which $(\Phi, c)$ is the spatially-discrete travelling wave (1.9).

The main contribution in [6] is that we modified the approach of [1] that was discussed above in such a way that Fredholm properties can be transferred from the spatially-discrete operators $\mathcal{L}_{h \text {; unif }}$ to the fully-discrete operators $\mathcal{L}_{h, \Delta t}$. Besides the singular transition from a first-order derivative to a first-order difference, there is also a structural transition in play 
here. Indeed, for $c \Delta t \in h \mathbb{Q}$ the natural spatial domain for the function $v$ in (1.14) is only a discrete subset of $\mathbb{R}$. The ability to handle such structural bifurcations is a second strong indicator of the versatility of the spectral convergence approach.

Continuum regime In [4] we introduced the continuous arclength coordinate $\theta=\theta(x, t)$ that satisfies $\theta_{x}=\sqrt{1+u_{x}^{2}}$. Upon passing to the $(\theta, t)$ coordinate system by writing

$$
w(\theta, t)=u(x(\theta, t), t), \quad \gamma(\theta, t)=\sqrt{1-w_{\theta}(\theta, t)^{2}},
$$

we transformed (1.1) into the fully nonlinear non-local system

$$
w_{t}=\gamma^{-2} w_{\theta \theta}+\gamma^{2} g_{\mathrm{cub}}(w ; a)+w_{\theta} \int_{-}\left(\gamma^{-4} w_{\theta \theta}+g_{\mathrm{cub}}(w ; a)\right) w_{\theta \theta} .
$$

Here we recall the notation $\left[\int_{-} f\right](\theta)=\int_{-\infty}^{\tau} f\left(\theta^{\prime}\right) d \theta^{\prime}$.

Let us now write $\Psi_{*}$ for the arclength reparametrization of the PDE waveprofile $\Phi_{*}$ and introduce the expression

$$
\gamma_{*}(\tau)=\sqrt{1-\Psi_{*}^{\prime}(\tau)^{2}} .
$$

In Sect. 3 we show that this stretched profile $\Psi_{*}$ satisfies the ODE

$$
c_{*} \gamma_{*}^{-1} \Psi_{*}^{\prime}=\gamma_{*}^{-4} \Psi_{*}^{\prime \prime}+g_{\text {cub }}\left(\Psi_{*} ; a\right) .
$$

In particular, the useful identity

$$
\int_{-}\left(\gamma_{*}^{-4} \Psi_{*}^{\prime \prime}+g_{\mathrm{cub}}\left(\Psi_{*} ; a\right)\right) \Psi_{*}^{\prime \prime}=c_{*} \int_{-} \gamma_{*}^{-1} \Psi_{*}^{\prime} \Psi_{*}^{\prime \prime}=c_{*}\left(1-\gamma_{*}\right)
$$

allows us to conclude that

$$
c_{*} \Psi_{*}^{\prime}=\gamma_{*}^{-2} \Psi_{*}^{\prime \prime}+\gamma_{*}^{2} g_{\mathrm{cub}}\left(\Psi_{*} ; a\right)+\Psi_{*}^{\prime} \int_{-}\left(\gamma_{*}^{-4} \Psi_{*}^{\prime \prime}+g_{\mathrm{cub}}\left(\Psi_{*} ; a\right)\right) \Psi_{*}^{\prime \prime},
$$

which means that $w(\theta, t)=\Psi_{*}\left(\theta+c_{*} t\right)$ satisfies (1.16).

Linearizing the stretched travelling wave ODE (1.18) around $\Psi_{*}$, we obtain the operator

$$
\mathcal{L}_{\mathrm{cmp}} v=-c_{*} \gamma_{*}^{-3} v^{\prime}+\gamma_{*}^{-4} v^{\prime \prime}+4 \gamma_{*}^{-6} \Psi_{*}^{\prime} \Psi_{*}^{\prime \prime} v^{\prime}+g_{\text {cub }}^{\prime}\left(\Psi_{*} ; a\right) v
$$

that acts with respect to the computational coordinate $\tau$. In Sect. 3.2 we analyze this operator in some detail and recast it back into the original physical coordinates. In fact, we show that it is not equivalent to the standard linearization $\mathcal{L}_{\mathrm{tw}}$ introduced in (1.6). It contains an extra term related to the stretching procedure that vanishes when applied to $\partial_{\xi} \Phi_{*}$. On the other hand, in the limits $\tau \rightarrow \pm \infty$ the differences between $\mathcal{L}_{\mathrm{cmp}}$ and $\mathcal{L}_{\mathrm{tw}}$ disappear. The essential spectrum hence remains unchanged. In addition, we explicitly show that the kernel of $\mathcal{L}_{\mathrm{cmp}}$ is also one-dimensional.

On the other hand, the linear operator $\mathcal{L}_{*}$ associated to the linearization of (1.20) is given by

$$
\begin{aligned}
\mathcal{L}_{*} v= & -c_{*} v^{\prime}+\gamma_{*}^{-2} v^{\prime \prime}+2 \gamma_{*}^{-4} \Psi_{*}^{\prime} \Psi_{*}^{\prime \prime} v^{\prime}+\gamma_{*}^{2} g_{\mathrm{cub}}^{\prime}\left(\Psi_{*} ; a\right) v-2 \Psi_{*}^{\prime} g_{\mathrm{cub}}\left(\Psi_{*} ; a\right) v^{\prime} \\
& +\Psi_{*}^{\prime} \int_{-}\left[4 \gamma_{*}^{-6} \Psi_{*}^{\prime}\left(\Psi_{*}^{\prime \prime}\right)^{2} v^{\prime}+\gamma_{*}^{-4} \Psi_{*}^{\prime \prime} v^{\prime \prime}+g_{\mathrm{cub}}^{\prime}\left(\Psi_{*} ; a\right) \Psi_{*}^{\prime \prime} v\right] \\
& +\Psi_{*}^{\prime} \int_{-}\left(\gamma_{*}^{-4} \Psi_{*}^{\prime \prime}+g_{\mathrm{cub}}\left(\Psi_{*} ; a\right)\right) v^{\prime \prime}+v^{\prime} \int_{-}\left(\gamma_{*}^{-4} \Psi_{*}^{\prime \prime}+g_{\mathrm{cub}}\left(\Psi_{*} ; a\right)\right) \Psi_{*}^{\prime \prime} .
\end{aligned}
$$

Using (1.18)-(1.19), together with $\partial_{\tau}\left[\gamma_{*}^{-1} \Psi_{*}^{\prime}\right]=\gamma_{*}^{-3} \Psi_{*}^{\prime \prime}$, this definition can be conveniently rewritten as

$$
\mathcal{L}_{*} v=\gamma_{*}^{2} \mathcal{L}_{\mathrm{cmp}} v+\Psi_{*}^{\prime} \int_{-} \Psi_{*}^{\prime \prime} \mathcal{L}_{\mathrm{cmp}} v
$$


In Sect. 3.3 we study the integral transform present in (1.23), which allows us to transfer key properties of the operator $\mathcal{L}_{\mathrm{cmp}}$ to $\mathcal{L}_{*}$. Let us emphasize once again that this twisted structure is a direct consequence of the procedure that we used in [4] to eliminate the mesh-speed $x_{t}$ from our system.

The singular perturbation In this paper we study the linear operators $\mathcal{L}_{h}$ that arise by recasting the integral in (1.23) as a sum and replacing all the derivatives except $-c_{*} v^{\prime}$ by their appropriate discrete counterparts. The precise expression is provided in Sect. 2, but conceptually this procedure is similar to the transitions

$$
\mathcal{L}_{\text {tw }} \rightarrow \mathcal{L}_{h ; \text { unif }}, \quad \mathcal{L}_{h ; \text { unif }} \rightarrow \mathcal{L}_{h, \Delta t}
$$

that we discussed above.

Our main goal here is to establish Fredholm properties for the operators $\mathcal{L}_{h}$. In particular, we generalize the spectral convergence approach described above to understand the singular transition from $\mathcal{L}_{*}$ to $\mathcal{L}_{h}$. This is a delicate task, since the structure of the operators $\mathcal{L}_{h}$ is significantly more complicated than that of $\mathcal{L}_{h}$; unif. In particular, the integral transform and the non-autonomous coefficients generate several new terms that were not present in [1]. In addition, we extend the techniques to gain control on the second and third discrete derivatives of solutions to the system $\mathcal{L}_{h} v=f$.

Our approach hinges on the fact that the new terms can all be shown to be localized in an appropriate sense. Nevertheless, recalling the sequences $\left\{v_{j}\right\} \subset H^{1}$ and $\left\{w_{j}\right\} \subset L^{2}$ with $\left(\mathcal{L}_{h}+\delta\right) v_{j}=w_{j}$, we need to extract subsequences for which the discrete derivatives of $v_{j}$ also converge strongly on compact intervals. We accomplish this by carefully controlling the size of the second-order discrete derivatives. This requires frequent use of a discrete summation-by-parts procedure to isolate this derivative from the convoluted expressions.

Although we do not pursue this here, we do believe that the techniques developed in [11] could be merged with the tools developed in this paper. In this way we would also be able to handle systems of reaction-diffusion equations in the bistable regime. We are less confident about possible generalizations to monostable equations, but passing to suitably weighted function spaces would be the first step to take.

Overview This paper is organized as follows. Our main results are formulated in Sect. 2. In Sect. 3 we discuss the impact on the PDE wave $\left(\Phi_{*}, c_{*}\right)$ caused by the transition from the physical coordinates to the computational coordinates. We develop some basic tools that link discrete and continuous calculus in Sect. 4. We continue in Sect. 5 by obtaining preliminary estimates concerning some of the terms appearing in $\mathcal{L}_{h}$. We conclude in Sect. 6 by analyzing the full structure of the operators $\mathcal{L}_{h}$. This allows us to generalize the spectral convergence method to establish Fredholm properties for these operators.

\section{Main Results}

The main results of this paper concern adaptive-grid discretizations of the scalar PDE

$$
u_{t}=u_{x x}+g(u) \text {. }
$$

Throughout the paper, we assume that the nonlinearity $g$ satisfies the following standard bistability condition. 
(Hg) The nonlinearity $g: \mathbb{R} \rightarrow \mathbb{R}$ is $C^{3}$-smooth and has a bistable structure, in the sense that there exists a constant $0<a<1$ such that we have

$$
g(0)=g(a)=g(1)=0, \quad g^{\prime}(0)<0, \quad g^{\prime}(1)<0,
$$

together with

$$
g(u)<0 \text { for } u \in(0, a) \cup(1, \infty), \quad g(u)>0 \text { for } u \in(-\infty,-1) \cup(a, 1) .
$$

It is well-known that the PDE (2.1) admits a travelling wave solution that connects the two stable equilibria of $g$ [2]. The key requirement in our next assumption is that this wave is not stationary, which can be arranged by demanding $\int_{0}^{1} g(u) d u \neq 0$.

$\left(\mathrm{H} \Phi_{*}\right)$ There exists a wave speed $c_{*} \neq 0$ and a profile $\Phi_{*} \in C^{5}(\mathbb{R}, \mathbb{R})$ that satisfies the limits

$$
\lim _{\xi \rightarrow-\infty} \Phi_{*}(\xi)=0, \quad \lim _{\xi \rightarrow+\infty} \Phi_{*}(\xi)=1
$$

and yields a solution to the PDE (2.1) upon writing

$$
u(x, t)=\Phi_{*}\left(x+c_{*} t\right) .
$$

\subsection{Computational Coordinates}

The physical wave coordinate $\xi=x+c_{*} t$ appearing in $\left(\mathrm{H} \Phi_{*}\right)$ is not well-suited for our purposes here, since we wish to work in the computational frame induced by the adaptive grid described in [4]. In order to compensate for this, we introduce the arclength

$$
\mathcal{A}(\xi)=\int_{0}^{\xi} \sqrt{1+\left[\partial_{\xi^{\prime}} \Phi_{*}\left(\xi^{\prime}\right)\right]^{2}} d \xi^{\prime} .
$$

Lemma 2.1 For every $\tau \in \mathbb{R}$, there is a unique $\xi_{*}(\tau)$ for which

$$
\mathcal{A}\left(\xi_{*}(\tau)\right)=\tau \text {. }
$$

Proof The existence of the right-inverse $\xi_{*}$ for $\mathcal{A}$ follows from

$$
\partial_{\xi} \mathcal{A}(\xi)=\sqrt{1+\left[\partial_{\xi} \Phi_{*}(\xi)\right]^{2}} \geq 1 .
$$

We are now in a position to introduce the stretched waveprofile $\Psi_{*}: \mathbb{R} \rightarrow \mathbb{R}$ that is given by

$$
\Psi_{*}(\tau)=\Phi_{*}\left(\xi_{*}(\tau)\right) .
$$

This profile $\Psi_{*}$ can be seen as the arclength parametrization of the graph of the physical wave $\Phi_{*}$. Upon introducing the notation

$$
\gamma_{*}(\tau)=\sqrt{1-\left[\partial_{\tau} \Psi_{*}\right](\tau)^{2}}=\sqrt{1-\Psi_{*}^{\prime}(\tau)^{2}},
$$

we will see in Sect. 3 that $\Psi_{*}$ satisfies the ODE

$$
c_{*} \gamma_{*}^{-1} \Psi_{*}^{\prime}=\gamma_{*}^{-4} \Psi_{*}^{\prime \prime}+g\left(\Psi_{*}\right) .
$$


It is hence natural to consider the linearized operator $\mathcal{L}_{\mathrm{cmp}}: H^{2} \rightarrow L^{2}$ associated to this system, which is given by

$$
\mathcal{L}_{\mathrm{cmp}} v=-c_{*} \gamma_{*}^{-3} v^{\prime}+\gamma_{*}^{-4} v^{\prime \prime}+4 \gamma_{*}^{-6} \Psi_{*}^{\prime} \Psi_{*}^{\prime \prime} v^{\prime}+g^{\prime}\left(\Psi_{*}\right) v .
$$

The formal adjoint $\mathcal{L}_{\mathrm{cmp}}^{\mathrm{adj}}: H^{2} \rightarrow L^{2}$ of this operator acts as

$$
\mathcal{L}_{\mathrm{cmp}}^{\mathrm{adj}} w=c_{*} \partial_{\tau}\left[\gamma_{*}^{-3} w\right]+\partial_{\tau \tau}\left[\gamma_{*}^{-4} w\right]-\partial_{\tau}\left[4 \gamma_{*}^{-6} \Psi_{*}^{\prime} \Psi_{*}^{\prime \prime} w\right]+g^{\prime}\left(\Psi_{*}\right) w
$$

Indeed, one may easily verify that for any pair $(v, w) \in H^{2} \times H^{2}$ we have

$$
\left\langle\mathcal{L}_{\mathrm{cmp}} v, w\right\rangle_{L^{2}}=\left\langle v, \mathcal{L}_{\mathrm{cmp}}^{\mathrm{adj}} w\right\rangle_{L^{2}} .
$$

As we have see in Sect. 1, the linearization of (1.16) leads naturally to a twisted version of $\mathcal{L}_{\mathrm{cmp}}$. To account for this, we introduce the notation

$$
\left[\int_{-} f\right](\tau)=\int_{-\infty}^{\tau} f\left(\tau^{\prime}\right) d \tau^{\prime}, \quad\left[\int_{+} f\right](\tau)=\int_{\tau}^{\infty} f\left(\tau^{\prime}\right) d \tau^{\prime}
$$

for the bounded continuous functions that arise after integrating a function $f \in L^{1}$. For any $f \in L^{2}$, this allows us to define the integral transform

$$
\mathcal{T}_{*} f=\gamma_{*}^{-2}\left[f-\gamma_{*} \Psi_{*}^{\prime} \int_{-} \gamma_{*}^{-3} \Psi_{*}^{\prime \prime} f\right],
$$

which can be inverted (see Sect. 3.3) by writing

$$
\mathcal{T}_{*}^{-1} w=\gamma_{*}^{2} w+\Psi_{*}^{\prime} \int_{-} \Psi_{*}^{\prime \prime} w .
$$

Finally, we introduce the function

$$
\Psi_{*}^{\operatorname{adj}}(\tau)=\left[\int \gamma_{*}^{-1}\left(\tau^{\prime}\right) \Psi_{*}^{\prime}\left(\tau^{\prime}\right) e^{-\int_{0}^{\tau^{\prime}} c_{*} \gamma_{*}(s) d s} \Psi_{*}^{\prime}\left(\tau^{\prime}\right) d \tau^{\prime}\right]^{-1} e^{-\int_{0}^{\tau} c_{*} \gamma_{*}(s) d s} \Psi_{*}^{\prime}(\tau),
$$

which in view of the computation

$$
\begin{aligned}
\mathcal{T}_{*} \Psi_{*}^{\prime} & =\gamma_{*}^{-2}\left[\Psi_{*}^{\prime}-\gamma_{*} \Psi_{*}^{\prime} \int_{-} \gamma_{*}^{-3} \Psi_{*}^{\prime \prime} \Psi_{*}^{\prime}\right] \\
& =\gamma_{*}^{-2}\left[\Psi_{*}^{\prime}-\gamma_{*} \Psi_{*}^{\prime}\left[\gamma_{*}^{-1}-1\right]\right] \\
& =\gamma_{*}^{-1} \Psi_{*}^{\prime}
\end{aligned}
$$

yields the non-standard normalization condition

$$
\left\langle\Psi_{*}^{\mathrm{adj}}, \mathcal{T}_{*} \Psi_{*}^{\prime}\right\rangle=1
$$

This choice is motivated by the following result, which allows us to interpret $\lambda=0$ as a simple eigenvalue for the twisted eigenvalue problem

$$
\mathcal{L}_{\mathrm{cmp}} v=\lambda \mathcal{T}_{*} v .
$$

Proposition 2.2 (see Sect. 3.2) Suppose that $(H g)$ and $\left(H \Phi_{*}\right)$ both hold. Then the operators $\mathcal{L}_{\mathrm{cmp}}: H^{2} \rightarrow L^{2}$ and $\mathcal{L}_{\mathrm{cmp}}^{\mathrm{adj}}: H^{2} \rightarrow L^{2}$ are both Fredholm with index zero. In addition, we have the identities

$$
\operatorname{Ker}\left(\mathcal{L}_{\mathrm{cmp}}\right)=\operatorname{span}\left\{\Psi_{*}^{\prime}\right\}, \quad \operatorname{Ker}\left(\mathcal{L}_{\mathrm{cmp}}^{\mathrm{adj}}\right)=\operatorname{span}\left\{\Psi_{*}^{\mathrm{adj}}\right\} .
$$




\subsection{Adaptive Linearization}

As a preparation, for any $v \in H^{1}$ we introduce the first-order differences

$$
\begin{aligned}
& {\left[\partial_{h}^{+} v\right](\tau)=h^{-1}[v(\tau+h)-v(\tau)],} \\
& {\left[\partial_{h}^{-} v\right](\tau)=h^{-1}[v(\tau)-v(\tau-h)],} \\
& {\left[\partial_{h}^{0} v\right](\tau)=(2 h)^{-1}[v(\tau+h)-v(\tau-h)],}
\end{aligned}
$$

together with the second-order counterpart

$$
\left[\partial_{h}^{(2)} v\right](\tau)=\left[\partial^{+} \partial^{-} v\right](\tau)=h^{-2}[v(\tau+h)+v(\tau-h)-2 v(\tau)] .
$$

In addition, we introduce the sums

$$
\left[\sum_{-; h} v\right](\tau)=h \sum_{k>0} v(\tau-k h), \quad\left[\sum_{+; h} v\right](\tau)=h \sum_{k>0} v(\tau+k h)
$$

and the notation

$$
\gamma_{h}=\sqrt{1-\left(\partial_{h}^{0} \Psi_{*}\right)^{2}}
$$

Finally, for any $v \in H^{1}$ and $h>0$, we introduce the function

$$
M_{h}[v]=-c_{*} \gamma_{h}^{-1} \partial_{h}^{0} v+4 \gamma_{h}^{-4} \partial_{h}^{0} \Psi_{*}\left[\partial_{h}^{(2)} \Psi_{*}\right] \partial_{h}^{0} v+\gamma_{h}^{-2} \partial_{h}^{(2)} v+\gamma_{h}^{2} g^{\prime}\left(\Psi_{*}\right) v .
$$

With this notation in hand, we are now ready to introduce the linear operators $\mathcal{L}_{h}: H^{1} \rightarrow$ $L^{2}$. These operators act as

$$
\mathcal{L}_{h} v=-c_{*} v^{\prime}+c_{*} \partial_{h}^{0} v+M_{h}[v]+\partial_{h}^{0} \Psi_{*} \sum_{-; h} \gamma_{h}^{-2}\left[\partial_{h}^{(2)} \Psi_{*}\right] M_{h}[v]
$$

and are the main focus of this paper. We will show in [5] that these operators can be seen in an appropriate sense as linearizations of the full adaptive mesh problem [4, Eq. (2.25)] around the stretched wave profile $\Psi_{*}$. Taking the limit $h \downarrow 0$, we see that $M_{h}$ formally reduces to $\gamma_{*}^{2} \mathcal{L}_{\text {cmp }}$. In particular, this means that $\mathcal{L}_{h}$ formally reduces to $\mathcal{T}_{*}^{-1} \mathcal{L}_{\text {cmp }}$ when taking $h \downarrow 0$.

Our main result provides a quasi-inverse for $\mathcal{L}_{h}$ that bifurcates off a twisted version of the operator $\mathcal{L}_{\mathrm{cmp}}$ discussed in Sect. 2.1. This accounts for the presence in (iii) of the integral transform $\mathcal{T}_{*}$. The crucial point in (i) is that we also obtain control on the $L^{2}$-norm of the second discrete derivative of $v$. This is slightly weaker than full $H^{2}$-control of $v$, but turns out to be sufficient to bound our nonlinear terms. In addition, item (ii) allows us to control an extra discrete derivative of $v$ provided one is available for $f$.

Theorem 2.3 (see Sect. 6) Suppose that $(H g)$ and $\left(H \Phi_{*}\right)$ are satisfied. Then there exist constants $K>0$ and $h_{0}>0$ together with linear maps

$$
\beta_{h}^{*}: L^{2} \rightarrow \mathbb{R}, \quad \mathcal{V}_{h}^{*}: L^{2} \rightarrow H^{1},
$$

defined for all $h \in\left(0, h_{0}\right)$, so that the following properties hold true.

(i) For all $f \in L^{2}$ and $0<h<h_{0}$, we have the bound

$$
\left|\beta_{h}^{*} f\right|+\left\|\mathcal{V}_{h}^{*} f\right\|_{H^{1}}+\left\|\partial_{h}^{+} \partial_{h}^{+} \mathcal{V}_{h}^{*} f\right\|_{L^{2}} \leq K\|f\|_{L^{2}} .
$$

(ii) For all $f \in L^{2}$ and $0<h<h_{0}$, we have the bound

$$
\left\|\partial_{h}^{+} \mathcal{V}_{h}^{*} f\right\|_{H^{1}}+\left\|\partial_{h}^{+} \partial_{h}^{+} \partial_{h}^{+} \mathcal{V}_{h}^{*} f\right\|_{L^{2}} \leq K\left[\|f\|_{L^{2}}+\left\|\partial_{h}^{+} f\right\|_{L^{2}}\right] .
$$


(iii) For all $f \in L^{2}$ and $0<h<h_{0}$, the pair

$$
(\beta, v)=\left(\beta_{h}^{*} f, \mathcal{V}_{h}^{*} f\right) \in \mathbb{R} \times H^{1}
$$

is the unique solution to the problem

$$
\mathcal{L}_{h} v=f+\beta \Psi_{*}^{\prime}
$$

that satisfies the normalization condition

$$
\left\langle\Psi_{*}^{\mathrm{adj}}, \mathcal{T}_{*} v\right\rangle_{L^{2}}=0 .
$$

(iv) We have $\beta_{h}^{*} \Psi_{*}^{\prime}=-1$ for all $h \in\left(0, h_{0}\right)$.

\section{Stretched PDE Waves}

We recall the functions $\mathcal{A}(\xi)$ and $\xi_{*}$ introduced in Lemma 2.1, which are related to the arclength parametrization of $\Phi_{*}$. We also recall the pair $\left(\Psi_{*}, \gamma_{*}\right)$ introduced in (2.9) and (2.10). Our first main result shows that $\gamma_{*}$ is well-defined and that it can be used to translate the travelling wave equation for the continuum model (2.1) into the stretched computational coordinates.

Proposition 3.1 Suppose that $(H g)$ and $\left(H \Phi_{*}\right)$ are satisfied. Then we have $\Psi_{*} \in C^{5}(\mathbb{R}, \mathbb{R})$ and there exists $\kappa>0$ so that the bounds

$$
0<\Psi_{*}^{\prime}(\tau)<1-\kappa, \quad \sqrt{\kappa}<\gamma_{*}(\tau)<1
$$

hold for all $\tau \in \mathbb{R}$. In addition, there exists a constant $K>0$ together with exponents $\eta_{-}>\max \left\{0, c_{*}\right\}$ and $\eta_{+}>\max \left\{0,-c_{*}\right\}$ for which the bound

$$
\left|\Psi_{*}(\tau)\right|+\left|\Psi_{*}^{\prime}(\tau)\right|+\left|\Psi_{*}^{\prime \prime}(\tau)\right|+\left|\Psi_{*}^{\prime \prime \prime}(\tau)\right|+\left|\Psi_{*}^{(i v)}(\tau)\right|+\left|\Psi_{*}^{(v)}(\tau)\right| \leq K e^{-\eta_{-}|\tau|}
$$

holds whenever $\tau<0$, while the bound

$$
\left|1-\Psi_{*}(\tau)\right|+\left|\Psi_{*}^{\prime}(\tau)\right|+\left|\Psi_{*}^{\prime \prime}(\tau)\right|+\left|\Psi_{*}^{\prime \prime \prime}(\tau)\right|+\left|\Psi_{*}^{(i v)}(\tau)\right|+\left|\Psi_{*}^{(v)}(\tau)\right| \leq K e^{-\eta_{+}|\tau|}
$$

holds for all $\tau \geq 0$. Finally, for every $\tau \in \mathbb{R}$ we have the identity

$$
c_{*} \gamma_{*}^{-1}(\tau) \Psi_{*}^{\prime}(\tau)=\gamma_{*}^{-4}(\tau) \Psi_{*}^{\prime \prime}(\tau)+g\left(\Psi_{*}(\tau)\right),
$$

together with the differentiated version

$$
\begin{aligned}
c_{*} \gamma_{*}^{-3}(\tau) \Psi_{*}^{\prime \prime}(\tau)= & \gamma_{*}^{-4}(\tau) \Psi_{*}^{\prime \prime \prime}(\tau)+4 \gamma_{*}^{-6}(\tau) \Psi_{*}^{\prime \prime}(\tau) \Psi_{*}^{\prime}(\tau) \Psi_{*}^{\prime \prime}(\tau) \\
& +g^{\prime}\left(\Psi_{*}(\tau)\right) \Psi_{*}^{\prime}(\tau) .
\end{aligned}
$$

The second main result in this section is an extended version of Proposition 2.2. In particular, we recall the linear operators (2.12)-(2.13) and obtain an essential estimate on the behaviour of $\left[\mathcal{L}_{\mathrm{cmp}}-\delta \mathcal{T}_{*}\right]^{-1}$ as $\delta \downarrow 0$. This will allow us to transfer the Fredholm properties of $\mathcal{L}_{\mathrm{cmp}}$ to its discrete twisted counterpart in Sect. 6. As a preparation, we introduce the adjoint integral transform $\mathcal{T}_{*}^{\text {adj }}$ that acts as

$$
\mathcal{T}_{*}^{\text {adj }} f=\gamma_{*}^{-2}\left[f-\gamma_{*}^{-1} \Psi_{*}^{\prime \prime} \int_{+} \gamma_{*}^{-1} \Psi_{*}^{\prime} f\right]
$$

for any $f \in L^{2}$. 
Proposition 3.2 The assumptions $(\mathrm{Hg})$ and $\left(H \Phi_{*}\right)$ imply the following properties.

(i) The operators $\mathcal{L}_{\mathrm{cmp}}: H^{2} \rightarrow L^{2}$ and $\mathcal{L}_{\mathrm{cmp}}^{\mathrm{adj}}: H^{2} \rightarrow L^{2}$ are both Fredholm with index zero and satisfy the identities

$$
\operatorname{Ker}\left(\mathcal{L}_{\mathrm{cmp}}\right)=\operatorname{span}\left\{\Psi_{*}^{\prime}\right\}, \quad \operatorname{Ker}\left(\mathcal{L}_{\mathrm{cmp}}^{\mathrm{adj}}\right)=\left\{\Psi_{*}^{\mathrm{adj}}\right\} .
$$

(ii) The linear maps $\mathcal{L}_{\mathrm{cmp}}-\delta \mathcal{T}_{*}$ and $\mathcal{L}_{\mathrm{cmp}}^{\mathrm{adj}}-\delta \mathcal{T}_{*}^{\text {adj }}$ are both invertible from $H^{2}$ into $L^{2}$ for all sufficiently small $\delta>0$.

(iii) There exists $K>0$ so that the bounds

$$
\| \begin{aligned}
& {\left[\mathcal{L}_{\mathrm{cmp}}-\delta \mathcal{T}_{*}\right]^{-1} f+\delta^{-1} \Psi_{*}^{\prime}\left\langle\Psi_{*}^{\mathrm{adj}}, f\right\rangle_{L^{2}}\left\|_{H^{2}} \leq K\right\| f \|_{L^{2}},} \\
& {\left[\mathcal{L}_{\mathrm{cmp}}^{\mathrm{adj}}-\delta \mathcal{T}_{*}^{\mathrm{adj}}\right]^{-1} f+\delta^{-1} \Psi_{*}^{\mathrm{adj}}\left\langle\Psi_{*}^{\prime}, f\right\rangle_{L^{2}}\left\|_{H^{2}} \leq K\right\| f \|_{L^{2}}}
\end{aligned}
$$

hold for all $f \in L^{2}$ and all sufficiently small $\delta>0$.

\subsection{Coordinate Transformation}

Consider two functions $f_{\text {cmp }}: \mathbb{R} \rightarrow \mathbb{R}$ and $f_{\text {phys }}: \mathbb{R} \rightarrow \mathbb{R}$. We introduce the stretching operator $\mathcal{S}_{*}$ and the compression operator $\mathcal{S}_{*}^{-1}$ that act as

$$
\left[\mathcal{S}_{*} f_{\text {phys }}\right](\tau)=f_{\text {phys }}\left(\xi_{*}(\tau)\right), \quad\left[\mathcal{S}_{*}^{-1} f_{\text {cmp }}\right](\xi)=f_{\text {cmp }}(\mathcal{A}(\xi)) .
$$

In particular, for any $\tau \in \mathbb{R}$ and $\xi \in \mathbb{R}$ we have the identities

$$
\left[\mathcal{S}_{*}^{-1} f_{\mathrm{cmp}}\right]\left(\xi_{*}(\tau)\right)=f_{\mathrm{cmp}}(\tau), \quad\left[\mathcal{S}_{*} f_{\text {phys }}\right](\mathcal{A}(\xi))=f_{\text {phys }}(\xi) .
$$

In order to understand the effect of these coordinate transformations on integrals and derivatives, we first need to understand $\xi_{*}^{\prime}$.

Lemma 3.3 Suppose that $(H g)$ and $\left(H \Phi_{*}\right)$ are satisfied. Then we have $\xi_{*} \in C^{1}(\mathbb{R} ; \mathbb{R})$. In addition, for any $\tau \in \mathbb{R}$ we have

$$
\xi_{*}^{\prime}(\tau)=\left[1+\left[\partial_{\xi} \Phi_{*}\left(\xi_{*}(\tau)\right)\right]^{2}\right]^{-1 / 2}=\gamma_{*}(\tau) .
$$

Proof The first identity in (3.11) follows by differentiating $\tau=\mathcal{A}\left(\xi_{*}(\tau)\right)$ with respect to $\tau$. Using the chain rule we compute

$$
\begin{aligned}
\Psi_{*}^{\prime}(\tau) & =\partial_{\tau}\left[\Phi_{*}\left(\xi_{*}(\tau)\right)\right] \\
& =\left[\partial_{\xi} \Phi_{*}\right]\left(\xi_{*}(\tau)\right) \xi_{*}^{\prime}(\tau) \\
& =\left[\partial_{\xi} \Phi_{*}\right]\left(\xi_{*}(\tau)\right)\left[1+\partial_{\xi} \Phi_{*}\left(\xi_{*}(\tau)\right)^{2}\right]^{-1 / 2}
\end{aligned}
$$

Squaring this identity yields

$$
\Psi_{*}^{\prime}(\tau)^{2}=1-\left[1+\partial_{\xi} \Phi_{*}\left(\xi_{*}(\tau)\right)^{2}\right]^{-1},
$$

which gives

$$
\left[1+\partial_{\xi} \Phi_{*}\left(\xi_{*}(\tau)\right)^{2}\right]^{-1}=1-\Psi_{*}^{\prime}(\tau)^{2}=\gamma_{*}(\tau)^{2},
$$

as desired.

를 Springer 
Corollary 3.4 Suppose that $(H g)$ and $\left(H \Phi_{*}\right)$ are satisfied. Then for any $f_{\mathrm{cmp}} \in C(\mathbb{R}, \mathbb{R}) \cap L^{2}$ and $f_{\text {phys }} \in C(\mathbb{R}, \mathbb{R}) \cap L^{2}$ we have the identity

$$
\left\langle f_{\text {phys }}, \mathcal{S}_{*}^{-1} f_{\text {cmp }}\right\rangle_{L^{2}}=\left\langle\mathcal{S}_{*} f_{\text {phys }}, \gamma_{*} f_{\mathrm{cmp}}\right\rangle_{L^{2}},
$$

together with

$$
\left\langle\mathcal{S}_{*} f_{\text {phys }}, f_{\mathrm{cmp}}\right\rangle_{L^{2}}=\left\langle f_{\text {phys }}, \mathcal{S}_{*}^{-1}\left[\gamma_{*}^{-1} f_{\mathrm{cmp}}\right]\right\rangle_{L^{2}} .
$$

In particular, $\mathcal{S}_{*}$ and $\mathcal{S}_{*}^{-1}$ can be interpreted as elements of $\mathcal{L}\left(L^{2} ; L^{2}\right)$.

Proof The substitution rule allows us to compute

$$
\begin{aligned}
\left\langle f_{\text {phys }}, \mathcal{S}_{*}^{-1} f_{\text {cmp }}\right\rangle_{L^{2}} & =\int f_{\text {phys }}(\xi) f_{\text {cmp }}(\mathcal{A}(\xi)) d \xi \\
& =\int f_{\text {phys }}\left(\xi_{*}(\tau)\right) f_{\text {cmp }}\left(\mathcal{A}\left(\xi_{*}(\tau)\right)\right) \xi_{*}^{\prime}(\tau) d \tau \\
& =\int f_{\text {phys }}\left(\xi_{*}(\tau)\right) f_{\text {cmp }}(\tau) \gamma_{*}(\tau) d \tau \\
& =\left\langle\mathcal{S}_{*} f_{\text {phys }}, \gamma_{*} f_{\text {cmp }}\right\rangle_{L^{2}} .
\end{aligned}
$$

The second identity follows in a similar fashion.

Corollary 3.5 Suppose that $(\mathrm{Hg})$ and $\left(H \Phi_{*}\right)$ are satisfied. Then for any $f_{\mathrm{cmp}} \in H^{1}$, we have $\mathcal{S}_{*}^{-1} f_{\mathrm{cmp}} \in H^{1}$ with

$$
\partial_{\xi}\left[\mathcal{S}_{*}^{-1} f_{\mathrm{cmp}}\right]=\mathcal{S}_{*}^{-1}\left[\gamma_{*}^{-1} \partial_{\tau} f_{\mathrm{cmp}}\right] .
$$

In addition, for any $f_{\text {phys }} \in H^{1}$, we have $\mathcal{S}_{*} f_{\text {phys }} \in H^{1}$ with

$$
\partial_{\tau}\left[\mathcal{S}_{*} f_{\text {phys }}\right]=\gamma_{*} \mathcal{S}_{*}\left[\partial_{\xi} f_{\text {phys }}\right] .
$$

Proof For $f_{\mathrm{cmp}} \in C^{1}(\mathbb{R} ; \mathbb{R})$ we may use the chain rule to compute

$$
\partial_{\xi}\left[f_{\mathrm{cmp}}(\mathcal{A}(\xi))\right]=\left[\partial_{\tau} f_{\mathrm{cmp}}\right](\mathcal{A}(\xi)) \partial_{\xi} \mathcal{A}(\xi)=\left[\partial_{\tau} f_{\mathrm{cmp}}\right](\mathcal{A}(\xi))\left[\xi_{*}^{\prime}(\mathcal{A}(\xi))\right]^{-1} .
$$

In addition, for $f_{\text {phys }} \in C^{1}(\mathbb{R} ; \mathbb{R})$ we compute

$$
\partial_{\tau}\left[f_{\text {phys }}\left(\xi_{*}(\tau)\right)\right]=\left[\partial_{\xi} f_{\text {phys }}\right]\left(\xi_{*}(\tau)\right) \xi_{*}^{\prime}(\tau) .
$$

The desired identities now follow from (3.9), (3.10) and (3.11). The final remark in Corollary 3.4 can be used to extend these results to $f_{\text {cmp }} \in H^{1}$ and $f_{\text {phys }} \in H^{1}$.

The physical wave $\Phi_{*}$ satisfies the travelling wave ODE

$$
c_{*} \partial_{\xi} \Phi_{*}(\xi)=\partial_{\xi \xi} \Phi_{*}(\xi)+g\left(\Phi_{*}(\xi)\right)
$$

for all $\xi \in \mathbb{R}$. It is well known that the limiting behaviour of $\Phi_{*}$ as $\xi \rightarrow \pm \infty$ depends on the roots of the characteristic functions

$$
\Delta_{ \pm}(\eta)=-c_{*} \eta+\eta^{2}+g^{\prime}\left(\Phi_{*}( \pm \infty)\right)
$$

In particular, upon writing

$$
\eta_{-}=\frac{1}{2} c_{*}+\frac{1}{2} \sqrt{c_{*}^{2}-4 g^{\prime}(0)}>\frac{1}{2} c_{*}+\frac{1}{2}\left|c_{*}\right|
$$

together with

$$
\eta_{+}=-\left[\frac{1}{2} c_{*}-\frac{1}{2} \sqrt{c_{*}^{2}-4 g^{\prime}(1)}\right]>-\frac{1}{2} c_{*}+\frac{1}{2}\left|c_{*}\right|
$$


and picking a sufficiently large $K>0$, we have the bounds

$$
\left|\partial_{\xi} \Phi_{*}(\xi)\right| \leq K e^{-\eta_{ \pm}|\xi|}
$$

for $\xi \in \mathbb{R}_{ \pm}$. In order to transfer this exponential bound to $\Psi_{*}^{\prime}$, we need to understand the differences $\xi_{*}(\tau)-\tau$.

Lemma 3.6 Suppose that $(H g)$ and $\left(H \Phi_{*}\right)$ are satisfied. Then there exists $K>0$ so that the inequality

$$
\left|\xi_{*}(\tau)-\tau\right|<K
$$

holds for any $\tau \in \mathbb{R}$.

Proof For any $x \in \mathbb{R}$ we have the standard inequality

$$
\sqrt{1+x^{2}}-1 \leq \frac{1}{2} x^{2}
$$

In particular, we see that

$$
\begin{aligned}
|\mathcal{A}(\xi)-\xi| & \leq \frac{1}{2} \int_{0}^{\xi} \partial_{\xi^{\prime}} \Phi_{*}\left(\xi^{\prime}\right)^{2} d \xi^{\prime} \\
& \leq \frac{1}{2}\left\|\partial_{\xi} \Phi_{*}\right\|_{L^{2}}^{2},
\end{aligned}
$$

which gives

$$
\left|\xi_{*}(\tau)-\tau\right|=\left|\xi_{*}(\tau)-\mathcal{A}\left(\xi_{*}(\tau)\right)\right| \leq \frac{1}{2}\left\|\partial_{\xi} \Phi_{*}\right\|_{L^{2}}^{2}
$$

Proof of Proposition 3.1 Using $\Phi_{*}=\mathcal{S}_{*}^{-1} \Psi_{*}$ together with the commutation relation

$$
g\left(\mathcal{S}_{*}^{-1} \Psi_{*}\right)=\mathcal{S}_{*}^{-1} g\left(\Psi_{*}\right),
$$

we can apply Corollary 3.5 to the travelling wave ODE (3.22) to obtain

$$
c_{*} \mathcal{S}_{*}^{-1}\left[\gamma_{*}^{-1} \Psi_{*}^{\prime}\right]=\mathcal{S}_{*}^{-1}\left[\gamma_{*}^{-1} \partial_{\tau}\left[\gamma_{*}^{-1} \Psi_{*}^{\prime}\right]\right]+\mathcal{S}_{*}^{-1}\left[g\left(\Psi_{*}\right)\right] .
$$

Using the identity

$$
\gamma_{*}^{\prime}=-\gamma_{*}^{-1} \Psi_{*}^{\prime} \Psi_{*}^{\prime \prime}
$$

together with the definition $\gamma_{*}^{2}=1-\left[\Psi_{*}^{\prime}\right]^{2}$, this gives

$$
\begin{aligned}
c_{*} \gamma_{*}^{-1} \Psi_{*}^{\prime} & =\gamma_{*}^{-2} \Psi_{*}^{\prime \prime}+\gamma_{*}^{-4}\left[\Psi_{*}^{\prime}\right]^{2} \Psi_{*}^{\prime \prime}+g\left(\Psi_{*}\right) \\
& =\gamma_{*}^{-4} \Psi_{*}^{\prime \prime}+g\left(\Psi_{*}\right) .
\end{aligned}
$$

A further differentiation yields

$$
c_{*} \gamma_{*}^{-1} \Psi_{*}^{\prime \prime}+c_{*} \gamma_{*}^{-3} \Psi_{*}^{\prime} \Psi_{*}^{\prime} \Psi_{*}^{\prime \prime}=\gamma_{*}^{-4} \Psi_{*}^{\prime \prime \prime}+4 \gamma_{*}^{-6} \Psi_{*}^{\prime \prime} \Psi_{*}^{\prime} \Psi_{*}^{\prime \prime}+g^{\prime}\left(\Psi_{*}\right) \Psi_{*}^{\prime},
$$

which can be simplified to (3.5).

The exponential bounds (3.2)-(3.3) now follow from Lemma 3.6 and (3.26), using (3.4) and its derivatives to understand the derivatives of order two and higher for $\Psi_{*}^{(i)}(\tau)$ for $2 \leq i \leq 5$. The inequality (3.1) for $\Psi_{*}^{\prime}$ follows directly from (3.13) and the fact that $\partial_{\xi} \Phi_{*}$ is uniformly bounded. Finally, the inequalities (3.1) for $\gamma_{*}$ follow from

$$
1>\sqrt{1-\Psi_{*}^{\prime}(\tau)^{2}}>\sqrt{1-(1-\kappa)^{2}}=\sqrt{2 \kappa-\kappa^{2}}>\sqrt{\kappa} .
$$




\subsection{Linear Operators}

In principle, most of the statements in Proposition 3.2(i) can be obtained by an appeal to standard Sturm-Liouville theory. We pursue a more explicit approach here in the hope that it can play a role towards generalizing the theory developed in this paper to non-scalar systems.

Our first two results highlight the fact that our coordinate transformation does not simply map $\mathcal{L}_{\mathrm{cmp}}$ and $\mathcal{L}_{\mathrm{cmp}}^{\mathrm{adj}}$ onto the standard linear operators

$$
\begin{aligned}
& \mathcal{L}_{\mathrm{tw}} y=-c_{*} \partial_{\xi} y+\partial_{\xi \xi} y+g^{\prime}\left(\Phi_{*}\right) y, \\
& \mathcal{L}_{\mathrm{tw}}^{\mathrm{adj}} z=+c_{*} \partial_{\xi} z+\partial_{\xi \xi} z+g^{\prime}\left(\Phi_{*}\right) z
\end{aligned}
$$

obtained by linearizing the travelling wave ODE (3.22) around $\Phi_{*}$. Indeed, the correct operators to consider are given by

$$
\begin{aligned}
& \mathcal{L}_{\text {phys }} y=\mathcal{L}_{\mathrm{tw}} y+\left(\partial_{\xi} \Phi_{*}\right)^{2} \frac{\partial_{\xi \xi} \Phi_{*}}{1+\left(\partial_{\xi} \Phi_{*}\right)^{2}} \partial_{\xi}\left[\frac{y}{\partial_{\xi} \Phi_{*}}\right] \\
& \mathcal{L}_{\text {phys }}^{\mathrm{adj}} z=\mathcal{L}_{\mathrm{tw}}^{\mathrm{adj}} z-\frac{1}{\partial_{\xi} \Phi_{*}} \partial_{\xi}\left[\left(\partial_{\xi} \Phi_{*}\right)^{2} \frac{\partial_{\xi \xi} \Phi_{*}}{1+\left(\partial_{\xi} \Phi_{*}\right)^{2}} z\right] .
\end{aligned}
$$

Lemma 3.7 Suppose that $(\mathrm{Hg})$ and $\left(H \Phi_{*}\right)$ are satisfied. Then for any $v \in H^{2}$ we have the identity

$$
\mathcal{L}_{\mathrm{cmp}} v=\gamma_{*}^{-1} \mathcal{S}_{*} \mathcal{L}_{\mathrm{phys}} \mathcal{S}_{*}^{-1}\left[\gamma_{*}^{-1} v\right] .
$$

Proof We write $y=\mathcal{S}_{*}^{-1}\left[\gamma_{*}^{-1} v\right]$, so that $\gamma_{*}^{-1} v=\mathcal{S}_{*} y$. Using Corollary 3.5 we get

$$
\begin{aligned}
\mathcal{S}_{*} \partial_{\xi} y & =\gamma_{*}^{-1} \partial_{\tau}\left[\gamma_{*}^{-1} v\right] \\
& =\gamma_{*}^{-4} \Psi_{*}^{\prime} \Psi_{*}^{\prime \prime} v+\gamma_{*}^{-2} v^{\prime} .
\end{aligned}
$$

In particular, (3.5) allows us to write

$$
c_{*} \mathcal{S}_{*} \partial_{\xi} y=c_{*} \gamma_{*}^{-2} v^{\prime}+\gamma_{*}^{-5} \Psi_{*}^{\prime} \Psi_{*}^{\prime \prime \prime} v+4 \gamma_{*}^{-7}\left(\Psi_{*}^{\prime}\right)^{2}\left(\Psi_{*}^{\prime \prime}\right)^{2} v+\gamma_{*}^{-1} g^{\prime}\left(\Psi_{*}\right)\left(1-\gamma_{*}^{2}\right) v .
$$

In addition, we compute

$$
\begin{aligned}
\mathcal{S}_{*} \partial_{\xi \xi} y= & \gamma_{*}^{-1} \partial_{\tau}\left[\mathcal{S}_{*} \partial_{\xi} y\right] \\
= & 4 \gamma_{*}^{-7}\left(\Psi_{*}^{\prime}\right)^{2}\left(\Psi_{*}^{\prime \prime}\right)^{2} v+\gamma_{*}^{-5}\left(\Psi_{*}^{\prime \prime} \Psi_{*}^{\prime \prime}+\Psi_{*}^{\prime} \Psi_{*}^{\prime \prime \prime}\right) v+\gamma_{*}^{-5} \Psi_{*}^{\prime} \Psi_{*}^{\prime \prime} v^{\prime} \\
& +2 \gamma_{*}^{-5} \Psi_{*}^{\prime} \Psi_{*}^{\prime \prime} v^{\prime}+\gamma_{*}^{-3} v^{\prime \prime} .
\end{aligned}
$$

We hence see

$$
\begin{aligned}
\gamma_{*}^{-1} \mathcal{S}_{*} \mathcal{L}_{\mathrm{tw}} y & =-c_{*} \gamma_{*}^{-3} v^{\prime}+\gamma_{*}^{-6}\left(\Psi_{*}^{\prime \prime}\right)^{2} v+3 \gamma_{*}^{-6} \Psi_{*}^{\prime} \Psi_{*}^{\prime \prime} v^{\prime}+\gamma_{*}^{-4} v^{\prime \prime}+g^{\prime}\left(\Psi_{*}\right) v \\
& =\mathcal{L}_{\mathrm{cmp}} v+\gamma_{*}^{-6}\left(\Psi_{*}^{\prime \prime}\right)^{2} v-\gamma_{*}^{-6} \Psi_{*}^{\prime} \Psi_{*}^{\prime \prime} v^{\prime} .
\end{aligned}
$$

We now write

$$
\mathcal{L}_{\text {phys }} y=\mathcal{L}_{\text {tw }} y+\partial_{\xi} \Phi_{*} \frac{\partial_{\xi \xi} \Phi_{*}}{1+\left(\partial_{\xi} \Phi_{*}\right)^{2}} \partial_{\xi} y-\frac{\left(\partial_{\xi \xi} \Phi_{*}\right)^{2}}{1+\left(\partial_{\xi} \Phi_{*}\right)^{2}} y .
$$

Exploiting the identities

$$
\begin{aligned}
\mathcal{S}_{*}\left[\partial_{\xi} \Phi_{*}\right] & =\gamma_{*}^{-1} \Psi_{*}^{\prime}, \\
\mathcal{S}_{*}\left[1+\left(\partial_{\xi} \Phi_{*}\right)^{2}\right] & =\gamma_{*}^{-2}, \\
\mathcal{S}_{*}\left[\partial_{\xi \xi} \Phi_{*}\right] & =\gamma_{*}^{-4} \Psi_{*}^{\prime \prime}
\end{aligned}
$$


together with (3.40), we may compute

$$
\begin{aligned}
\gamma_{*}^{-1} \mathcal{S}_{*} \mathcal{L}_{\mathrm{phys}} y= & \gamma_{*}^{-1} \mathcal{S}_{*} \mathcal{L}_{\mathrm{tw}} y+\gamma_{*}^{-4} \Psi_{*}^{\prime} \Psi_{*}^{\prime \prime}\left[\gamma_{*}^{-4} \Psi_{*}^{\prime} \Psi_{*}^{\prime \prime} v+\gamma_{*}^{-2} v^{\prime}\right] \\
& -\gamma_{*}^{-7}\left(\Psi_{*}^{\prime \prime}\right)^{2}\left[\gamma_{*}^{-1} v\right] \\
= & \gamma_{*}^{-1} \mathcal{S}_{*} \mathcal{L}_{\mathrm{tw}} y-\gamma_{*}^{-6}\left(\Psi_{*}^{\prime \prime}\right)^{2} v+\gamma_{*}^{-6} \Psi_{*}^{\prime} \Psi_{*}^{\prime \prime} v^{\prime} \\
= & \mathcal{L}_{\mathrm{cmp}} v,
\end{aligned}
$$

as desired.

Lemma 3.8 Suppose that $(H g)$ and $\left(H \Phi_{*}\right)$ are satisfied. Then for any $w \in H^{2}$ we have the identity

$$
\mathcal{L}_{\mathrm{cmp}}^{\mathrm{adj}} w=\mathcal{S}_{*} \mathcal{L}_{\mathrm{phys}}^{\mathrm{adj}} \mathcal{S}_{*}^{-1}\left[\gamma_{*}^{-2} w\right] .
$$

Proof Pick $v \in H^{2}$. Applying Corollary 3.4 twice, we compute

$$
\begin{aligned}
& \left\langle\mathcal{L}_{\mathrm{cmp}} v, w\right\rangle_{L^{2}}=\left\langle\gamma_{*}^{-1} \mathcal{S}_{*} \mathcal{L}_{\mathrm{phys}} \mathcal{S}_{*}^{-1} \gamma_{*}^{-1} v, w\right\rangle_{L^{2}} \\
& =\left\langle\mathcal{S}_{*} \mathcal{L}_{\text {phys }} \mathcal{S}_{*}^{-1} \gamma_{*}^{-1} v, \gamma_{*}^{-1} w\right\rangle_{L^{2}} \\
& =\left\langle\mathcal{L}_{\text {phys }} \mathcal{S}_{*}^{-1} \gamma_{*}^{-1} v, \mathcal{S}_{*}^{-1}\left[\gamma_{*}^{-2} w\right]\right\rangle_{L^{2}} \\
& =\left\langle\mathcal{S}_{*}^{-1} \gamma_{*}^{-1} v, \mathcal{L}_{\text {phys }}^{\text {adj }} \mathcal{S}_{*}^{-1}\left[\gamma_{*}^{-2} w\right]\right\rangle_{L^{2}} \\
& =\left\langle v, \mathcal{S}_{*} \mathcal{L}_{\text {phys }}^{\text {adj }} \mathcal{S}_{*}^{-1}\left[\gamma_{*}^{-2} w\right]\right\rangle_{L^{2}} .
\end{aligned}
$$

The result now follows from (2.14).

The explicit form (3.38) allows one to immediately verify that

$$
\mathcal{L}_{\text {phys }} \partial_{\xi} \Phi_{*}=\mathcal{L}_{\text {tw }} \partial_{\xi} \Phi_{*}=0 .
$$

Upon defining

$$
\Phi_{*}^{\mathrm{adj} ; \mathrm{tw}}(\xi)=e^{-c_{*} \xi} \partial_{\xi} \Phi_{*}(\xi),
$$

it is a standard exercise to verify that $\mathcal{L}_{\mathrm{tw}}^{\mathrm{adj}} \Phi_{*}^{\mathrm{adj} ; \mathrm{tw}}=0$. We now construct a kernel element for $\mathcal{L}_{\text {phys }}^{\text {adj }}$ by writing

$$
\Phi_{*}^{\text {adj;phys }}(\xi)=\sqrt{1+\left(\partial_{\xi} \Phi_{*}(\xi)\right)^{2}} \Phi_{*}^{\text {adj; tw }}(\xi) .
$$

Lemma 3.9 Suppose that $(H g)$ and $\left(H \Phi_{*}\right)$ are satisfied. Then we have

$$
\mathcal{L}_{\text {phys }}^{\text {adj }} \Phi_{*}^{\text {adj;phys }}=0 .
$$

Proof We first compute

$$
\begin{aligned}
\mathcal{L}_{\mathrm{tw}}^{\mathrm{adj}} \Phi_{*}^{\mathrm{adj} ; \text { phys }}= & c_{*} \frac{\partial_{\xi} \Phi_{*} \partial_{\xi \xi} \Phi_{*}}{\sqrt{1+\left(\partial_{\xi} \Phi_{*}\right)^{2}}} \Phi_{*}^{\mathrm{adj} ; \mathrm{tw}}+\partial_{\xi}\left[\frac{\partial_{\xi} \Phi_{*} \partial_{\xi \xi} \Phi_{*}}{\sqrt{1+\left(\partial_{\xi} \Phi_{*}\right)^{2}}}\right] \Phi_{*}^{\mathrm{adj} ; \mathrm{tw}} \\
& +2 \frac{\partial_{\xi} \Phi_{*} \partial_{\xi \xi} \Phi_{*}}{\sqrt{1+\left(\partial_{\xi} \Phi_{*}\right)^{2}}} \partial_{\xi} \Phi_{*}^{\mathrm{adj} ; \mathrm{tw}}
\end{aligned}
$$

Upon writing

$$
\mathcal{I}=\frac{1}{\partial_{\xi} \Phi_{*}} \partial_{\xi}\left[\left(\partial_{\xi} \Phi_{*}\right)^{2} \frac{\partial_{\xi \xi} \Phi_{*}}{1+\left(\partial_{\xi} \Phi_{*}\right)^{2}} \Phi_{*}^{\text {adj; phys }}\right]
$$

we also compute

$$
\begin{aligned}
\mathcal{I} & =\frac{1}{\partial_{\xi} \Phi_{*}} \partial_{\xi}\left[\left(\partial_{\xi} \Phi_{*}\right)^{2} \frac{\partial_{\xi \xi} \Phi_{*}}{\sqrt{1+\left(\partial_{\xi} \Phi_{*}\right)^{2}}} \Phi_{*}^{\mathrm{adj} ; \mathrm{tw}}\right] \\
& =\partial_{\xi}\left[\frac{\partial_{\xi} \Phi_{*} \partial_{\xi \xi} \Phi_{*}}{\sqrt{1+\left(\partial_{\xi} \Phi_{*}\right)^{2}}}\right] \Phi_{*}^{\mathrm{adj} ; \mathrm{tw}}+\frac{\left(\partial_{\xi \xi} \Phi_{*}\right)^{2}}{\sqrt{1+\left(\partial_{\xi} \Phi_{*}\right)^{2}}} \Phi_{*}^{\mathrm{adj} ; \mathrm{tw}}+\frac{\partial_{\xi} \Phi_{*} \partial_{\xi \xi} \Phi_{*}}{\sqrt{1+\left(\partial_{\xi} \Phi_{*}\right)^{2}}} \partial_{\xi} \Phi_{*}^{\mathrm{adj} ; \mathrm{tw}} .
\end{aligned}
$$


In particular, we find

$$
\mathcal{L}_{\text {phys }}^{\text {adj }} \Phi_{*}^{\text {adj;phys }}=c_{*} \frac{\partial_{\xi} \Phi_{*} \partial_{\xi \xi} \Phi_{*}}{\sqrt{1+\left(\partial_{\xi} \Phi_{*}\right)^{2}}} \Phi_{*}^{\text {adj;tw }}+\frac{\partial_{\xi} \Phi_{*} \partial_{\xi \xi} \Phi_{*}}{\sqrt{1+\left(\partial_{\xi} \Phi_{*}\right)^{2}}} \partial_{\xi} \Phi_{*}^{\mathrm{adj} ; \mathrm{tw}}-\frac{\left(\partial_{\xi \xi} \Phi_{*}\right)^{2}}{\sqrt{1+\left(\partial_{\xi} \Phi_{*}\right)^{2}}} \Phi_{*}^{\mathrm{adj} ; \mathrm{tw}}
$$

The result now follows from the computation

$$
\begin{aligned}
\partial_{\xi} \Phi_{*} \partial_{\xi} \Phi^{\mathrm{adj} ; \mathrm{tw}} & =\partial_{\xi} \Phi_{*} \partial_{\xi}\left[e^{\left.-c_{*} \cdot \partial_{\xi} \Phi_{*}\right]}\right. \\
& =-c_{*} \partial_{\xi} \Phi_{*} \Phi^{\mathrm{adj} ; \mathrm{tw}}+\Phi^{\mathrm{adj} ; \mathrm{tw}} \partial_{\xi \xi} \Phi_{*} .
\end{aligned}
$$

Lemma 3.10 Suppose that $(H g)$ and $\left(H \Phi_{*}\right)$ are satisfied and recall the definition (2.18). Then the identity

$$
\Psi_{*}^{\text {adj }}=\left[\int \gamma_{*}^{-1}(\tau) \Psi_{*}^{\prime}(\tau) e^{-\int_{0}^{\tau} c_{*} \gamma_{*}(s) d s} \Psi_{*}^{\prime}(\tau) d \tau\right]^{-1} \gamma_{*}^{2} \mathcal{S}_{*}\left[\Phi_{*}^{\text {adj;phys }}\right]
$$

holds. In particular, the representation (3.47) implies that $\mathcal{L}_{\mathrm{cmp}}^{\mathrm{adj}} \Psi_{*}^{\mathrm{adj}}=0$.

Proof This follows directly from

$$
\begin{aligned}
& \mathcal{S}_{*}\left[\partial_{\xi} \Phi_{*}\right] \\
& \mathcal{S}_{*}\left[\sqrt{1+\left(\partial_{\xi} \Phi_{*}\right)^{2}}\right]=\gamma_{*}^{-1} \Psi_{*}^{\prime}, \\
& =\gamma_{*}^{-1},
\end{aligned}
$$

together with the computation

$$
\mathcal{S}_{*}\left[\xi \mapsto e^{-c_{*} \xi}\right](\tau)=e^{-c_{*} \xi_{*}(\tau)}=e^{-c_{*} \int_{0}^{\tau} \gamma_{*}(s) d s} .
$$

Here we used $\xi_{*}(0)=0$ and $\xi_{*}^{\prime}(s)=\gamma_{*}(s)$.

Lemma 3.11 Suppose that $(H g)$ and $\left(H \Phi_{*}\right)$ are satisfied. Then we have

$$
\operatorname{Ker} \mathcal{L}_{\text {phys }}=\operatorname{span}\left\{\Phi_{*}^{\prime}\right\} .
$$

Proof A potential second, linearly independent kernel element can be written as $\alpha \partial_{\xi} \Phi_{*}$ for some function $\alpha$. We hence compute

$$
\mathcal{L}_{\text {phys }}\left[\alpha \partial_{\xi} \Phi_{*}\right]=-c_{*} \partial_{\xi} \alpha \partial_{\xi} \Phi_{*}+\partial_{\xi \xi} \alpha \partial_{\xi} \Phi_{*}+2 \partial_{\xi} \alpha \partial_{\xi \xi} \Phi_{*}+\left(\partial_{\xi} \Phi_{*}\right)^{2} \frac{\partial_{\xi \xi} \Phi_{*}}{1+\left(\partial_{\xi} \Phi_{*}\right)^{2}} \partial_{\xi} \alpha .
$$

Setting the right hand side to zero, we find

$$
\begin{aligned}
\partial_{\xi \xi} \alpha & =\left[c_{*}-2 \frac{\partial_{\xi \xi} \Phi_{*}}{\partial_{\xi} \Phi_{*}}-\frac{\partial_{\xi} \Phi_{*} \partial_{\xi \xi} \Phi_{*}}{1+\left(\partial_{\xi} \Phi_{*}\right)^{2}}\right] \partial_{\xi} \alpha \\
& =\partial_{\xi}\left[c_{*} \xi-2 \ln \left[\partial_{\xi} \Phi_{*}\right]-\frac{1}{2} \ln \left[1+\left(\partial_{\xi} \Phi_{*}\right)^{2}\right]\right] \partial_{\xi} \alpha .
\end{aligned}
$$

Choosing an integration constant $\alpha_{*} \in \mathbb{R}$, this can be solved to yield

$$
\partial_{\xi} \alpha=\alpha_{*}\left(\partial_{\xi} \Phi_{*}\right)^{-2} e^{c_{*} \xi} \frac{1}{\sqrt{1+\left(\partial_{\xi} \Phi_{*}\right)^{2}}} .
$$

For $\alpha_{*} \neq 0$ it is clear that one can choose $\kappa>0$ in such a way that

$$
|\alpha(\xi)| \geq \kappa e^{2 \eta+\xi+c_{*} \xi}
$$

holds for all sufficiently large $\xi \gg 1$. This prevents $\alpha \partial_{\xi} \Phi_{*}$ from being bounded. 
Proof of Proposition 3.2(i) Viewing $\mathcal{L}_{\mathrm{cmp}}, \mathcal{L}_{\text {phys }}$ and $\mathcal{L}_{\mathrm{tw}}$ as operators in $\mathcal{L}\left(H^{2} ; L^{2}\right)$, we observe that their essential spectral are equal. Indeed, the differential equations arising in the $\xi \rightarrow \pm \infty$ and $\tau \rightarrow \pm \infty$ limits agree with each other. In particular, all these operators are Fredholm with index zero. The description of $\operatorname{Ker} \mathcal{L}_{\text {cmp }}$ follows directly from (3.61) and the correspondence (3.39). The description of $\operatorname{Ker} \mathcal{L}_{\mathrm{cmp}}^{\mathrm{adj}}$ follows directly from Lemma 3.10 and the fact that

$$
0=\operatorname{ind}\left(\mathcal{L}_{\mathrm{cmp}}\right)=\operatorname{dim}\left(\operatorname{Ker} \mathcal{L}_{\mathrm{cmp}}\right)-\operatorname{dim}\left(\operatorname{Ker} \mathcal{L}_{\mathrm{cmp}}^{\mathrm{adj}}\right)
$$

\subsection{Integral Transforms}

Our goals here are to discuss the integral transforms introduced in (2.16) and (3.6) and to prove items (ii) and (iii) of Proposition 3.2. In particular, the integral transforms can be used to solve two integral equations that appear naturally when linearizing the adaptive grid equations around the stretched wave $\Psi_{*}$.

Lemma 3.12 Suppose that $(H g)$ and $\left(H \Phi_{*}\right)$ are satisfied. There exists $K>0$ so that the bound

$$
\left\|\mathcal{T}_{*} f\right\|_{L^{2}} \leq K\|f\|_{L^{2}}
$$

holds for any $f \in L^{2}$, while the bound

$$
\left\|\mathcal{T}_{*}^{\text {adj }} f\right\|_{H^{2}} \leq K\|f\|_{H^{2}}
$$

holds for all $f \in H^{2}$.

Proof The estimate (3.67) follows from the uniform bound (3.1), together with the inclusion $\Psi_{*} \in H^{2}$ and the inequality

$$
\left\|\int_{-} \gamma_{*}^{-3} \Psi_{*}^{\prime \prime} f\right\|_{\infty} \leq\left\|\gamma_{*}^{-3}\right\|_{\infty}\left\|\Psi_{*}^{\prime \prime}\right\|_{L^{2}}\|f\|_{L^{2}} .
$$

Writing $w=\mathcal{T}_{*}^{\text {adj }} f$, we note that

$$
\begin{aligned}
w^{\prime}= & {\left[\gamma_{*}^{-2} f\right]^{\prime}-\left[\gamma_{*}^{-3} \Psi_{*}^{\prime \prime}\right]^{\prime} \int_{+} \gamma_{*}^{-1} \Psi_{*}^{\prime} f+\gamma_{*}^{-4} \Psi_{*}^{\prime \prime} \Psi_{*}^{\prime} f, } \\
w^{\prime \prime}= & {\left[\gamma_{*}^{-2} f\right]^{\prime \prime}-\left[\gamma_{*}^{-3} \Psi_{*}^{\prime \prime}\right]^{\prime \prime} \int_{+} \gamma_{*}^{-1} \Psi_{*}^{\prime} f+\left[\gamma_{*}^{-3} \Psi_{*}^{\prime \prime}\right]^{\prime} \gamma_{*}^{-1} \Psi_{*}^{\prime} f } \\
& +\left[\gamma_{*}^{-4} \Psi_{*}^{\prime \prime} \Psi_{*}^{\prime}\right]^{\prime} f+\gamma_{*}^{-4} \Psi_{*}^{\prime \prime} \Psi_{*}^{\prime} f^{\prime} .
\end{aligned}
$$

Exploiting the inclusion $\Psi_{*} \in H^{4}$ and the bound

$$
\left\|\int_{+} \gamma_{*}^{-1} \Psi_{*}^{\prime} f\right\|_{\infty} \leq\left\|\gamma_{*}^{-1}\right\|_{\infty}\left\|\Psi_{*}^{\prime}\right\|_{L^{2}}\|f\|_{L^{2}},
$$

we see that indeed $w \in H^{2}$ and that the estimate (3.68) holds.

Lemma 3.13 Consider any pair $(w, f) \in L^{2} \times L^{2}$. Then the identity

$$
\gamma_{*}^{2} w+\Psi_{*}^{\prime} \int_{-} \Psi_{*}^{\prime \prime} w=f
$$


holds if and only if

$$
w=\mathcal{T}_{*} f=\gamma_{*}^{-2} f-\gamma_{*}^{-1} \Psi_{*}^{\prime} \int_{-} \gamma_{*}^{-3} \Psi_{*}^{\prime \prime} f .
$$

Proof Assuming (3.72) holds, we write

$$
X=\int_{-} \Psi_{*}^{\prime \prime} w
$$

and compute

$$
X^{\prime}=\Psi_{*}^{\prime \prime} w=\gamma_{*}^{-2} \Psi_{*}^{\prime \prime} f-\gamma_{*}^{-2} \Psi_{*}^{\prime} \Psi_{*}^{\prime \prime} X .
$$

Recalling $\gamma_{*}^{\prime}=-\gamma_{*}^{-1} \Psi_{*}^{\prime} \Psi_{*}^{\prime \prime}$, we see that

$$
\left[\gamma_{*}^{-1} X\right]^{\prime}=\gamma_{*}^{-3} \Psi_{*}^{\prime \prime} f
$$

Using the fact that $X(\tau) \rightarrow 0$ as $\tau \rightarrow-\infty$, this implies

$$
X=\gamma_{*} \int_{-} \gamma_{*}^{-3} \Psi_{*}^{\prime \prime} f
$$

and hence

$$
\gamma_{*}^{2} w=f-\Psi_{*}^{\prime} X=f-\gamma_{*} \Psi_{*}^{\prime} \int_{-} \gamma_{*}^{-3} \Psi_{*}^{\prime \prime} f .
$$

On the other hand, assuming (3.73), we compute

$$
\begin{aligned}
\int_{-} \Psi_{*}^{\prime \prime} w & =\int_{-} \gamma_{*}^{-2} \Psi_{*}^{\prime \prime} f-\int_{-}\left[\gamma_{*}^{-1} \Psi_{*}^{\prime \prime} \Psi_{*}^{\prime} \int_{-} \gamma_{*}^{-3} \Psi_{*}^{\prime \prime} f\right] \\
& =\int_{-} \gamma_{*}^{-2} \Psi_{*}^{\prime \prime} f+\int_{-}\left[\gamma_{*}^{\prime} \int_{-} \gamma_{*}^{-3} \Psi_{*}^{\prime \prime} f\right] \\
& =\int_{-} \gamma_{*}^{-2} \Psi_{*}^{\prime \prime} f+\gamma_{*} \int_{-} \gamma_{*}^{-3} \Psi_{*}^{\prime \prime} f-\int_{-} \gamma_{*} \gamma_{*}^{-3} \Psi_{*}^{\prime \prime} f \\
& =\gamma_{*} \int_{-} \gamma_{*}^{-3} \Psi_{*}^{\prime \prime} f .
\end{aligned}
$$

Multiplying by $\Psi_{*}^{\prime}$, we hence see

$$
\Psi_{*}^{\prime} \int_{-} \Psi_{*}^{\prime \prime} w=\gamma_{*} \Psi_{*}^{\prime} \int_{-} \gamma_{*}^{-3} \Psi_{*}^{\prime \prime} f=f-\gamma_{*}^{2} w,
$$

which yields (3.72).

Lemma 3.14 Consider any pair $(w, f) \in H^{2} \times H^{2}$. Then the identity

$$
\gamma_{*}^{2} w+\Psi_{*}^{\prime \prime} \int_{+} \Psi_{*}^{\prime} w=f
$$

holds if and only if

$$
w=\mathcal{T}_{*}^{\text {adj }} f=\gamma_{*}^{-2}\left[f-\gamma_{*}^{-1} \Psi_{*}^{\prime \prime} \int_{+} \gamma_{*}^{-1} \Psi_{*}^{\prime} f\right] .
$$

Proof Assuming (3.81) holds, we write

$$
Y=\int_{+} \Psi_{*}^{\prime} w
$$

and compute

$$
Y^{\prime}=-\Psi_{*}^{\prime} w=-\Psi_{*}^{\prime} \gamma_{*}^{-2} f+\gamma_{*}^{-2} \Psi_{*}^{\prime} \Psi_{*}^{\prime \prime} Y .
$$


In particular, we see that

$$
\left[\gamma_{*} Y\right]^{\prime}=-\gamma_{*}^{-1} \Psi_{*}^{\prime} f
$$

We hence find

$$
Y=\gamma_{*}^{-1} \int_{+} \gamma_{*}^{-1} \Psi_{*}^{\prime} f
$$

which yields

$$
w=\gamma_{*}^{-2}\left[f-\Psi_{*}^{\prime \prime} Y\right]=\gamma_{*}^{-2}\left[f-\gamma_{*}^{-1} \Psi_{*}^{\prime \prime} \int_{+} \gamma_{*}^{-1} \Psi_{*}^{\prime} f\right] .
$$

On the other hand, assuming (3.82) we compute

$$
\begin{aligned}
\int_{+} \Psi_{*}^{\prime} w & =\int_{+} \gamma_{*}^{-2} \Psi_{*}^{\prime} f-\int_{+}\left[\gamma_{*}^{-3} \Psi_{*}^{\prime} \Psi_{*}^{\prime \prime} \int_{+} \gamma_{*}^{-1} \Psi_{*}^{\prime} f\right] \\
& =\int_{+} \gamma_{*}^{-2} \Psi_{*}^{\prime} f-\int_{+}\left[\left[\gamma_{*}^{-1}\right]^{\prime} \int_{+} \gamma_{*}^{-1} \Psi_{*}^{\prime} f\right] \\
& =\int_{+} \gamma_{*}^{-2} \Psi_{*}^{\prime} f+\left[\gamma_{*}^{-1}\right] \int_{+} \gamma_{*}^{-1} \Psi_{*}^{\prime} f-\int_{+} \gamma_{*}^{-1} \gamma_{*}^{-1} \Psi_{*}^{\prime} f \\
& =\gamma_{*}^{-1} \int_{+} \gamma_{*}^{-1} \Psi_{*}^{\prime} f .
\end{aligned}
$$

Multiplying by $\Psi_{*}^{\prime \prime}$, we find

$$
\Psi_{*}^{\prime \prime} \int_{+} \Psi_{*}^{\prime} w=\gamma_{*}^{-1} \Psi_{*}^{\prime \prime} \int_{+} \gamma_{*}^{-1} \Psi_{*}^{\prime} f=f-\gamma_{*}^{2} w
$$

which yields (3.81).

Proof of Proposition 3.2(ii)-(iii) We introduce the notation

$$
\alpha_{c}[f]=\left\langle\Psi_{*}^{\mathrm{adj}}, f\right\rangle_{L^{2}}
$$

and note that the normalization (2.20) implies that $\alpha_{c}\left[\mathcal{T}_{*} \Psi_{*}^{\prime}\right]=1$. In particular, the operator

$$
\pi_{c} f=\left[\mathcal{T}_{*} \Psi_{*}^{\prime}\right] \alpha_{c} f
$$

is a projection on $L^{2}$. Writing $\pi=I-\pi_{c}$, the Fredholm alternative (see e.g. [9, Thm. 2.2.1]) now yields the splitting $L^{2}=R \oplus R_{c}$ with

$$
R=\pi\left(L^{2}\right)=\mathcal{L}_{\mathrm{cmp}}\left(H^{2}\right), \quad R_{c}=\pi_{c}\left(L^{2}\right) .
$$

Upon choosing a splitting

$$
H^{2}=\operatorname{span}\left\{\Psi_{*}^{\prime}\right\} \oplus K_{c},
$$

we note that the linear map

$$
\mathcal{L}_{\mathrm{cmp}}: K_{c} \rightarrow R
$$

is invertible, which implies that the perturbed operators

$$
\left[\mathcal{L}_{\mathrm{cmp}}-\delta \pi \mathcal{T}_{*}\right]: K_{c} \rightarrow R
$$

are also invertible for small $\delta>0$. For any $f \in R$, we introduce the function

$$
L_{\delta}[f]=\left[\mathcal{L}_{\mathrm{cmp}}-\delta \pi \mathcal{T}_{*}\right]^{-1} f-\Psi_{*}^{\prime} \alpha_{c}\left[\mathcal{T}_{*}\left[\mathcal{L}_{\mathrm{cmp}}-\delta \pi \mathcal{T}_{*}\right]^{-1} f\right]
$$


and use the identity $\mathcal{L}_{\mathrm{cmp}} \Psi_{*}^{\prime}=0$ to compute

$$
\begin{aligned}
{\left[\mathcal{L}_{\mathrm{cmp}}-\delta \mathcal{T}_{*}\right] L_{\delta} f=} & {\left[\mathcal{L}_{\mathrm{cmp}}-\delta \mathcal{T}_{*}\right]\left[\mathcal{L}_{\mathrm{cmp}}-\delta \pi \mathcal{T}_{*}\right]^{-1} f+\delta \mathcal{T}_{*} \Psi_{*}^{\prime} \alpha_{c}\left[\mathcal{T}_{*}\left[\mathcal{L}_{\mathrm{cmp}}-\delta \pi \mathcal{T}_{*}\right]^{-1} f\right] } \\
= & f-\delta \pi_{c} \mathcal{T}_{*}\left[\mathcal{L}_{\mathrm{cmp}}-\delta \pi \mathcal{T}_{*}\right]^{-1} f+\delta \mathcal{T}_{*} \Psi_{*}^{\prime} \alpha_{c}\left[\mathcal{T}_{*}\left[\mathcal{L}_{\mathrm{cmp}}-\delta \pi \mathcal{T}_{*}\right]^{-1} f\right] \\
= & f-\delta \mathcal{T}_{*} \Psi_{*}^{\prime} \alpha_{c}\left[\mathcal{T}_{*}\left[\mathcal{L}_{\mathrm{cmp}}-\delta \pi \mathcal{T}_{*}\right]^{-1} f\right] \\
& +\delta \mathcal{T}_{*} \Psi_{*}^{\prime} \alpha_{c}\left[\mathcal{T}_{*}\left[\mathcal{L}_{\mathrm{cmp}}-\delta \pi \mathcal{T}_{*}\right]^{-1} f\right] \\
= & f .
\end{aligned}
$$

For any $f \in L^{2}$, this allows us to conclude

$$
\begin{aligned}
{\left[\mathcal{L}_{\mathrm{cmp}}-\delta \mathcal{T}_{*}\right]\left[-\delta^{-1} \Psi_{*}^{\prime} \alpha_{c}[f]+L_{\delta} \pi[f]\right] } & =\mathcal{T}_{*} \Psi_{*}^{\prime} \alpha_{c}[f]+\pi[f] \\
& =f,
\end{aligned}
$$

which provides an inverse for $\mathcal{L}_{\mathrm{cmp}}-\delta \mathcal{T}_{*}$. An analogous procedure can be used to obtain the result for $\mathcal{L}_{\mathrm{cmp}}^{\mathrm{adj}}$.

\section{Sampling Techniques}

In order to exploit the continuum theory developed in Sect. 3, we need to expand the results developed in [4, Sect. A] in order to allow for detailed comparisons between functions and their associated sampled sequences. In this section we collect several tools that will be useful for these procedures.

In Sect. 4.1 we obtain several useful results that relate the discrete operators $\partial_{h}^{ \pm}$and $\sum_{ \pm ; h}$ back to their continuous counterparts. In Sect. 4.2 we introduce exponentially weighted norms on $L^{2}$ and discuss their impact on the summed functions (2.25). Finally, in Sect. 4.3 we discuss sequences of differences (2.23) and sums (2.25) for which $h \downarrow 0$. Upon taking weak limits, it is possible to recover the usual continuous derivatives and integrals.

\subsection{Discrete Versus Continuous Calculus}

As a reminder, we recall the sequence spaces

$$
\begin{aligned}
& \ell_{h}^{2}=\left\{V: h \mathbb{Z} \rightarrow \mathbb{R} \text { for which }\|V\|_{\ell_{h}^{2}}^{2}:=h \sum_{j \in \mathbb{Z}}\left|V_{h j}\right|^{2}<\infty\right\}, \\
& \ell_{h}^{\infty}=\left\{V: h \mathbb{Z} \rightarrow \mathbb{R} \text { for which }\|V\|_{\ell_{h}^{\infty}}:=\sup _{j \in \mathbb{Z}}\left|V_{h j}\right|<\infty\right\}
\end{aligned}
$$

that were introduced in [4, Sect. 3.3]. Our goal here is to obtain error bounds in these spaces when applying differences and sums instead of derivatives and integrals to continuous functions. As a preparation, we repeat the useful estimates [4, Eqs. (A.6), (A.13)] which state that

$$
\|u\|_{\ell_{h}^{2}} \leq(2+h)\|u\|_{H^{1}}, \quad\left\|\partial_{h}^{ \pm} u\right\|_{L^{q}} \leq\left\|u^{\prime}\right\|_{L^{q}}
$$

for any $u \in H^{1}$ and $q \in\{2, \infty\}$.

Lemma 4.1 Pick $q \in\{2, \infty\}$ and consider any $u \in W^{2 ; q}$. Then the estimates

$$
\left\|\partial_{h}^{ \pm} u-u^{\prime}\right\|_{\ell_{h}^{q}} \leq h\left\|u^{\prime \prime}\right\|_{L^{q}}
$$

hold for all $h>0$. 
Proof Fix $h>0$ and write $\mathcal{I}^{ \pm} \in \ell_{h}^{\infty}$ for the sequences

$$
\mathcal{I}_{j h}^{ \pm}=\left[\partial_{h}^{ \pm} u\right](j h)-u^{\prime}(j h) .
$$

We may compute

$$
\begin{aligned}
\mathcal{I}_{j h}^{+} & =\frac{1}{h} \int_{0}^{h}\left[u^{\prime}(j h+s)-u^{\prime}(j h)\right] d s \\
& =\int_{0}^{1}\left[u^{\prime}(j h+s h)-u^{\prime}(j h)\right] d s \\
& =\int_{0}^{1} \int_{0}^{s h} u^{\prime \prime}\left(j h+s^{\prime}\right) d s^{\prime} d s .
\end{aligned}
$$

For $q=\infty$ we hence see

$$
\left|\mathcal{I}_{j h}^{+}\right| \leq\left\|u^{\prime \prime}\right\|_{L^{\infty}} \int_{0}^{1} \int_{0}^{s h} d s^{\prime} d s=\frac{1}{2} h\left\|u^{\prime \prime}\right\|_{L^{\infty}} .
$$

For $q=2$ we obtain the estimate

$$
\begin{aligned}
\left\|\mathcal{I}^{+}\right\|_{\ell_{h}^{2}}^{2} & =h \sum_{j \in \mathbb{Z}}\left[\int_{0}^{1} \int_{0}^{s h} u^{\prime \prime}\left(j h+s^{\prime}\right) d s^{\prime} d s\right]^{2} \\
& \leq h \sum_{j \in \mathbb{Z}} \int_{0}^{1}\left[\int_{0}^{s h} u^{\prime \prime}\left(j h+s^{\prime}\right) d s^{\prime}\right]^{2} d s \\
& \leq h \sum_{j \in \mathbb{Z}} \int_{0}^{1} s h \int_{0}^{s h}\left[u^{\prime \prime}\left(j h+s^{\prime}\right)\right]^{2} d s^{\prime} d s \\
& \leq h^{2} \sum_{j \in \mathbb{Z}} \int_{0}^{h}\left[u^{\prime \prime}\left(j h+s^{\prime}\right)\right]^{2} d s^{\prime} \\
& =h^{2}\left\|u^{\prime \prime}\right\|_{L^{2}}^{2} .
\end{aligned}
$$

Similar computations can be used for $\mathcal{I}^{-}$.

Corollary 4.2 Pick $q \in\{2, \infty\}$ and consider any $u \in W^{3 ; q}$. Then the estimates

$$
\begin{aligned}
& \left\|\partial_{h}^{(2)} u-u^{\prime \prime}\right\|_{\ell_{h}^{q}} \leq 2 h\left\|u^{\prime \prime \prime}\right\|_{L^{q}}, \\
& \left\|\partial_{h}^{(2)} u(\cdot+h)-u^{\prime \prime}\right\|_{\ell_{h}^{q}} \leq 2 h\left\|u^{\prime \prime \prime}\right\|_{L^{q}}
\end{aligned}
$$

hold for all $h>0$.

Proof We first compute

$$
\begin{aligned}
\partial_{h}^{(2)} u-u^{\prime \prime} & =\partial_{h}^{+} \partial_{h}^{-} u-u^{\prime \prime} \\
& =\partial_{h}^{+} \partial_{h}^{-} u-\partial_{h}^{-} u^{\prime}+\partial_{h}^{-} u^{\prime}-u^{\prime \prime} .
\end{aligned}
$$

Applying Lemma 4.1 and (4.2) to $\partial_{h}^{-} u$ shows that

$$
\left\|\partial_{h}^{+} \partial_{h}^{-} u-\partial_{h}^{-} u^{\prime}\right\|_{\ell_{h}^{q}} \leq h\left\|\partial_{h}^{-} u^{\prime \prime}\right\|_{L^{q}} \leq h\left\|u^{\prime \prime \prime}\right\|_{L^{q}} .
$$

Similarly, applying Lemma 4.1 to $u^{\prime}$ shows that

$$
\left\|\partial_{h}^{-} u^{\prime}-u^{\prime \prime}\right\|_{\ell_{h}^{q}} \leq h\left\|u^{\prime \prime \prime}\right\|_{L^{q}},
$$

from which the first estimate follows. Upon writing

$$
\begin{aligned}
\partial_{h}^{(2)} u(\cdot+h)-u^{\prime \prime} & =\partial_{h}^{+} \partial_{h}^{+} u-u^{\prime \prime} \\
& =\partial_{h}^{+} \partial_{h}^{+} u-\partial_{h}^{+} u^{\prime}+\partial_{h}^{+} u^{\prime}-u^{\prime \prime},
\end{aligned}
$$

the second estimate can be obtained in a similar fashion. 
Corollary 4.3 Pick $q \in\{2, \infty\}$ and consider any $u \in W^{4 ; q}$. Then the estimate

$$
\left\|\partial_{h}^{+} \partial_{h}^{(2)} u-u^{\prime \prime \prime}\right\|_{\ell_{h}^{q}} \leq 3 h\left\|u^{(i v)}\right\|_{L^{q}}
$$

holds for all $h>0$.

Proof Splitting up

$$
\begin{aligned}
\partial_{h}^{+} \partial_{h}^{(2)} u-u^{\prime \prime \prime}= & \partial_{h}^{+} \partial_{h}^{+} \partial_{h}^{-} u-u^{\prime \prime \prime} \\
= & \partial_{h}^{+} \partial_{h}^{+} \partial_{h}^{-} u-\partial_{h}^{+} \partial_{h}^{-} u^{\prime} \\
& +\partial_{h}^{+} \partial_{h}^{-} u^{\prime}-\partial_{h}^{-} u^{\prime \prime} \\
& +\partial_{h}^{-} u^{\prime \prime}-u^{\prime \prime \prime},
\end{aligned}
$$

we can apply Lemma 4.1 to obtain

$$
\left\|\partial_{h}^{+} \partial_{h}^{(2)} u-u^{\prime \prime \prime}\right\|_{\ell_{h}^{q}} \leq h\left\|\partial_{h}^{+} \partial_{h}^{-} u^{\prime \prime}\right\|_{L^{q}}+h\left\|\partial_{h}^{-} u^{\prime \prime \prime}\right\|_{L^{q}}+h\left\|u^{\prime \prime \prime \prime}\right\|_{L^{q}}
$$

We can now repeatedly apply (4.2) to obtain the desired estimate.

We recall the definitions (2.15). Our final result here is a standard approximation bound for discrete integration.

Lemma 4.4 For any $f \in W^{1,1}$ and $h>0$, we have the bounds

$$
\left\|\sum_{ \pm ; h} f-\int_{ \pm} f\right\|_{\ell_{h}^{\infty}} \leq h\left\|f^{\prime}\right\|_{L^{1}} .
$$

Proof Fixing $\tau \in \mathbb{R}$, we compute

$$
\begin{aligned}
{\left[\sum_{+; h} f-\int_{+} f\right](\tau) } & =\sum_{k>0} \int_{0}^{h}[f(\tau+k h)-f(\tau+(k-1) h+\sigma)] d \sigma \\
& =\sum_{k \geq 0} \int_{0}^{h}[f(\tau+(k+1) h)-f(\tau+k h+\sigma)] d \sigma \\
& =\sum_{k \geq 0} \int_{0}^{h} \int_{\sigma}^{h} f^{\prime}\left(\tau+k h+\sigma^{\prime}\right) d \sigma^{\prime} d \sigma \\
& =\sum_{k \geq 0} \int_{0}^{h} \int_{0}^{\sigma^{\prime}} f^{\prime}\left(\tau+k h+\sigma^{\prime}\right) d \sigma d \sigma^{\prime} .
\end{aligned}
$$

In particular, we obtain the estimate

$$
\begin{aligned}
\left|\left[\sum_{+; h} f-\int_{+} f\right](\tau)\right| & \leq \sum_{k \geq 0} h \int_{0}^{h}\left|f^{\prime}\left(\tau+k h+\sigma^{\prime}\right)\right| d \sigma^{\prime} \\
& \leq h \int_{0}^{\infty}\left|f^{\prime}\left(\tau+\sigma^{\prime}\right)\right| d \sigma^{\prime} \\
& \leq h\left\|f^{\prime}\right\|_{L^{1}} .
\end{aligned}
$$

\subsection{Weighted Norms}

For any $\eta>0$ we define the exponential weight function

$$
e_{\eta}(\tau)=e^{-\eta|\tau|} .
$$

This allows us to define an inner product

$$
\langle a, b\rangle_{L_{\eta}^{2}}=\left\langle e_{\eta} a, e_{\eta} b\right\rangle_{L^{2}}=\left\langle e_{2 \eta} a, b\right\rangle_{L^{2}},
$$


together with the associated Hilbert space

$$
L_{\eta}^{2}=\left\{f \in L_{\mathrm{loc}}^{1}:\|f\|_{L_{\eta}^{2}}^{2}:=\langle f, f\rangle_{L_{\eta}^{2}}<\infty\right\} .
$$

Since $0<e_{\eta} \leq 1$, we see that

$$
\langle a, a\rangle_{L_{\eta}^{2}} \leq\langle a, a\rangle_{L^{2}}
$$

for every $a \in L^{2}$. In particular, we have the continuous embedding

$$
L^{2} \subset L_{\eta}^{2} \text {. }
$$

In addition, for any pair $(a, b) \in L_{\eta}^{2} \times L^{2}$, we have $e_{\eta} a \in L^{2}$ and hence also $e_{2 \eta} a \in L^{2}$. This allows us to estimate

$$
\left|\left\langle e_{2 \eta} a, b\right\rangle_{L^{2}}\right|=\left|\langle a, b\rangle_{L_{\eta}^{2}}\right| \leq\|a\|_{L_{\eta}^{2}}\|b\|_{L_{\eta}^{2}} .
$$

This weighted norm is very convenient when dealing with sampling sums.

Lemma 4.5 Fix $\eta>0$. There exists $K>0$ so that for any $f \in L_{\eta}^{2}$ and any $0<h<1$, we have the estimate

$$
\left\|\sum_{-; h} e_{2 \eta} f\right\|_{L_{\eta}^{2}} \leq K\|f\|_{L_{\eta}^{2}}
$$

Proof Using Cauchy-Schwartz, we compute

$$
\begin{aligned}
\left\|\sum_{-; h} e_{2 \eta} f\right\|_{L_{\eta}^{2}}^{2} & =\int e_{2 \eta}(\tau)\left[\sum_{-; h} e_{2 \eta} f\right](\tau)^{2} d \tau \\
& =\int e_{2 \eta}(\tau)\left[h \sum_{k>0} e_{\eta}^{2}(\tau-k h) f(\tau-k h)\right]^{2} d \tau \\
& \leq \int e_{2 \eta}(\tau)\left[h \sum_{k>0} e_{2 \eta}(\tau-k h)\right]\left[h \sum_{k>0} e_{2 \eta}(\tau-k h) f(\tau-k h)^{2}\right] d \tau .
\end{aligned}
$$

We note that there exists $C_{1}>0$ so that for all $0<h \leq 1$ and all $\tau \in \mathbb{R}$ we have

$$
\begin{aligned}
h \sum_{k>0} e_{2 \eta}(\tau-k h) & =h \sum_{k>0} e^{-2 \eta|\tau-k h|} \\
& \leq h \sum_{k \in \mathbb{Z}} e^{-2 \eta|\tau-k h|} \\
& \leq C_{1} .
\end{aligned}
$$

Using the substitution $\tau^{\prime}=\tau-k h$, this allows us to compute

$$
\begin{aligned}
\left\|\sum_{-; h} e_{2 \eta} f\right\|_{L_{\eta}^{2}}^{2} & \leq C_{1} \int e_{2 \eta}(\tau)\left[h \sum_{k>0} e_{2 \eta}(\tau-k h) f(\tau-k h)^{2}\right] d \tau \\
& =C_{1} \int\left[h \sum_{k>0} e_{2 \eta}\left(\tau^{\prime}+k h\right)\right] e_{2 \eta}\left(\tau^{\prime}\right) f\left(\tau^{\prime}\right)^{2} d \tau^{\prime} \\
& \leq C_{1}^{2} \int e_{2 \eta}\left(\tau^{\prime}\right) f\left(\tau^{\prime}\right)^{2} d \tau^{\prime} \\
& =C_{1}^{2}\|f\|_{L_{\eta}^{2}}^{2} .
\end{aligned}
$$

\subsection{Weak Limits}

Our results here show how weak limits interact with discrete summation and differentiation. The first result concerns sequences that are bounded in $H^{1}$ and have bounded second differences, as described in the following assumption. 
(hSeq) The sequence

$$
\left\{\left(h_{j}, v_{j}\right)\right\}_{j>0} \subset(0,1) \times H^{1}
$$

satisfies $h_{j} \rightarrow 0$ as $j \rightarrow \infty$. In addition, there exists $K>0$ so that the bound

$$
\left\|v_{j}\right\|_{H^{1}}+\left\|\partial_{h_{j}}^{+} \partial_{h_{j}}^{+} v_{j}\right\|_{L^{2}}<K
$$

holds for all $j>0$.

The control on the second differences allows one to show that the weak limit is in fact in $H^{2}$. In addition, the first differences converge strongly on compact intervals.

Lemma 4.6 Consider a sequence

$$
\left\{\left(h_{j}, v_{j}\right)\right\} \subset(0,1) \times H^{1}
$$

that satisfies (hSeq). Then there exists $V_{*} \in H^{2}$ so that, after passing to a subsequence, the following properties hold.

(i) We have the weak limit

$$
v_{j} \rightarrow V_{*} \in H^{1}
$$

(ii) We have the weak limits

$$
\partial_{h_{j}}^{ \pm} v_{j} \rightarrow V_{*}^{\prime} \in L^{2}
$$

(iii) We have the weak limit

$$
\partial_{h_{j}}^{(2)} v_{j} \rightarrow V_{*}^{\prime \prime} \in L^{2} .
$$

(iv) For any compact interval $\mathcal{I} \subset \mathbb{R}$, we have the strong convergences

$$
v_{j} \rightarrow V_{*} \in L^{2}(\mathcal{I}), \quad \partial_{h_{j}}^{ \pm} v_{j} \rightarrow V_{*}^{\prime} \in L^{2}(\mathcal{I})
$$

as $j \rightarrow \infty$.

Proof Using (4.2) we obtain the uniform bound

$$
\left\|\partial_{h_{j}}^{ \pm} v_{j}\right\|_{L^{2}}<K
$$

for all $j>0$. In particular, after passing to a subsequence we can find a triplet

$$
\left(V_{*}, V_{*}^{ \pm}, V_{*}^{(2)}\right) \in H^{1} \times L^{2} \times L^{2}
$$

so that we have the weak convergences

$$
v_{j} \rightarrow V_{*} \in H^{1}, \quad \partial_{h_{j}}^{ \pm} v_{j} \rightarrow V_{*}^{ \pm} \in L^{2}, \quad \partial_{h_{j}}^{(2)} v_{j} \rightarrow V_{*}^{(2)} \in L^{2}
$$

as $j \rightarrow \infty$.

Pick any test function $\zeta \in C_{c}^{\infty}$. We note that

$$
\left\|\partial_{h_{j}}^{-} \zeta-\zeta^{\prime}\right\|_{L^{2}}+\left\|\partial_{h_{j}}^{(2)} \zeta-\zeta^{\prime \prime}\right\|_{L^{2}} \rightarrow 0
$$

as $j \rightarrow \infty$ by Lemma 4.1 and Corollary 4.2. 
We now compute

$$
\begin{aligned}
\left\langle\partial_{h_{j}}^{+} v_{j}, \zeta\right\rangle_{L^{2}} & =-\left\langle v_{j}, \partial_{h_{j}}^{-} \zeta\right\rangle_{L^{2}} \\
& =-\left\langle v_{j}, \zeta^{\prime}\right\rangle_{L^{2}}+\left\langle v_{j}, \zeta^{\prime}-\partial_{h_{j}}^{-} \zeta\right\rangle_{L^{2}} \\
& =\left\langle v_{j}^{\prime}, \zeta\right\rangle_{L^{2}}+\left\langle v_{j}, \zeta^{\prime}-\partial_{h_{j}}^{-} \zeta\right\rangle_{L^{2}},
\end{aligned}
$$

together with

$$
\begin{aligned}
\left\langle\partial_{h_{j}}^{(2)} v_{j}, \zeta\right\rangle_{L^{2}} & =\left\langle v_{j}, \partial_{h_{j}}^{(2)} \zeta\right\rangle_{L^{2}} \\
& =\left\langle v_{j}, \zeta^{\prime \prime}\right\rangle_{L^{2}}+\left\langle v_{j}, \partial_{h_{j}}^{(2)} \zeta-\zeta^{\prime \prime}\right\rangle_{L^{2}} \\
& =-\left\langle v_{j}^{\prime}, \zeta^{\prime}\right\rangle_{L^{2}}+\left\langle v_{j}, \partial_{h_{j}}^{(2)} \zeta-\zeta^{\prime \prime}\right\rangle_{L^{2}}
\end{aligned}
$$

The weak convergences $v_{j}^{\prime} \rightarrow V_{*}^{\prime} \in L^{2}$ and (4.38) imply that

$$
\begin{array}{ll}
\left\langle\partial_{h_{j}}^{+} v_{j}, \zeta\right\rangle_{L^{2}} \rightarrow\left\langle V_{*}^{\prime}, \zeta\right\rangle_{L^{2}}, & \left\langle\partial_{h_{j}}^{+} v_{j}, \zeta\right\rangle_{L^{2}} \rightarrow\left\langle V_{*}^{+}, \zeta\right\rangle_{L^{2}}, \\
\left\langle\partial_{h_{j}}^{(2)} v_{j}, \zeta\right\rangle_{L^{2}} \rightarrow-\left\langle V_{*}^{\prime}, \zeta^{\prime}\right\rangle_{L^{2}}, & \left\langle\partial_{h_{j}}^{(2)} v_{j}, \zeta\right\rangle_{L^{2}} \rightarrow\left\langle V_{*}^{(2)}, \zeta\right\rangle_{L^{2}}
\end{array}
$$

as $j \rightarrow \infty$. The density of $C_{c}^{\infty}$ in $L^{2}$ now implies that $V_{*}^{+}=V_{*}^{\prime}$ and that $V_{*}^{\prime} \in H^{1}$ with $V_{*}^{\prime \prime}=V_{*}^{(2)}$. This yields (i), (ii) and (iii).

Turning to (iv), we pick a compact interval $\mathcal{I} \subset \mathbb{R}$. The compact embedding $H^{1}(\mathcal{I}) \subset$ $L^{2}(\mathcal{I})$ allows us to pass to a subsequence for which

$$
\left\|v_{j}-V_{*}\right\|_{L^{2}(\mathcal{I})} \rightarrow 0
$$

as $j \rightarrow \infty$. We compute

$$
\begin{aligned}
\left\|\partial_{h_{j}}^{+} v_{j}-V_{*}^{\prime}\right\|_{L^{2}(\mathcal{I})}= & \left\langle\partial_{h_{j}}^{+} v_{j}-V_{*}^{\prime}, \partial_{h_{j}}^{+} v_{j}-V_{*}^{\prime}\right\rangle_{L^{2}(\mathcal{I})} \\
= & \left\langle\partial_{h_{j}}^{+} v_{j}-V_{*}^{\prime}, \partial_{h_{j}}^{+} v_{j}-\partial_{h_{j}}^{+} V_{*}\right\rangle_{L^{2}(\mathcal{I})}+\left\langle\partial_{h_{j}}^{+} v_{j}-V_{*}^{\prime}, \partial_{h_{j}}^{+} V_{*}-V_{*}^{\prime}\right\rangle_{L^{2}(\mathcal{I})} \\
= & -\left\langle\partial_{h_{j}}^{-} \partial_{h_{j}}^{+} v_{j}-\partial_{h_{j}}^{-} V_{*}^{\prime}, v_{j}-V_{*}\right\rangle_{L^{2}(\mathcal{I})} \\
& +\left\langle\partial_{h_{j}}^{+} v_{j}-V_{*}^{\prime}, \partial_{h_{j}}^{+} V_{*}-V_{*}^{\prime}\right\rangle_{L^{2}(\mathcal{I})} .
\end{aligned}
$$

Using (4.2) we see that

$$
\left\|\partial_{h_{j}}^{-} V_{*}^{\prime}\right\|_{L^{2}} \leq\left\|V_{*}^{\prime \prime}\right\|_{L^{2}} .
$$

Together with (4.30), (4.36) and the identity

$$
\partial_{h_{j}}^{-} \partial_{h_{j}}^{+} v_{j}=\partial_{h_{j}}^{(2)} v_{j}
$$

this implies the uniform bound

$$
\left\|\partial_{h_{j}}^{-} \partial_{h_{j}}^{+} v_{j}\right\|_{L^{2}(\mathcal{I})}+\left\|\partial_{h_{j}}^{+} v_{j}\right\|_{L^{2}(\mathcal{I})}+\left\|\partial_{h_{j}}^{-} V_{*}^{\prime}\right\|_{L^{2}(\mathcal{I})}+\left\|V_{*}^{\prime}\right\|_{L^{2}(\mathcal{I})}<C_{1}
$$

for some $C_{1}>0$. In particular, using Lemma 4.1 and (4.43), we see that

$$
\begin{aligned}
\left\|\partial_{h_{j}}^{+} v_{j}-V_{*}^{\prime}\right\|_{L^{2}(\mathcal{I})} & \leq 2 C_{1}\left[\left\|v_{j}-V_{*}\right\|_{L^{2}(\mathcal{I})}+\left\|\partial_{h_{j}}^{+} V_{*}-V_{*}^{\prime}\right\|_{L^{2}(\mathcal{I})}\right] \\
& \leq 2 C_{1}\left[\left\|v_{j}-V_{*}\right\|_{L^{2}(\mathcal{I})}+h_{j}\left\|V_{*}^{\prime \prime}\right\|_{L^{2}}\right] \\
& \rightarrow 0
\end{aligned}
$$

as $j \rightarrow \infty$, as desired. A standard diagonalization argument now completes the proof. 
Lemma 4.7 Consider a bounded sequence

$$
\left\{\left(h_{j}, f_{j}, \alpha_{1 ; j}, \alpha_{2 ; j}, \alpha_{3 ; j}\right)\right\}_{j>0} \subset(0,1) \times L^{2} \times H^{1} \times H^{1} \times H^{1}
$$

that satisfies the following properties.

(a) There exists $C>0$ and $\eta>0$ so that

$$
\left|\alpha_{1 ; j}(\tau)\right|+\left|\alpha_{2 ; j}(\tau)\right| \leq C e_{2 \eta}(\tau)
$$

for all $\tau>0$.

(b) There exists a triplet $\left(\alpha_{1 ; *}, \alpha_{2 ; *}, \alpha_{3 ; *}\right) \in H^{1} \times H^{1} \times H^{1}$ so that we have the strong convergence

$$
\left(\alpha_{1 ; j}, \alpha_{2 ; j}, \alpha_{3 ; j}\right) \rightarrow\left(\alpha_{1 ; *}, \alpha_{2 ; *}, \alpha_{3 ; *}\right) \in H^{1} \times H^{1} \times H^{1}
$$

as $j \rightarrow \infty$.

(c) We have $h_{j} \rightarrow 0$ as $j \rightarrow \infty$.

Then, after passing to a subsequence, there exists $f_{*} \in L^{2}$ so that we have the weak convergences

$$
f_{j} \rightarrow f_{*} \in L^{2}, \quad \alpha_{3 ; j} f_{j} \rightarrow \alpha_{3 ; *} \in L^{2},
$$

together with

$$
\alpha_{1 ; j} \sum_{-; h_{j}} \alpha_{2 ; j} f_{j} \rightarrow \alpha_{1 ; *} \int_{-} \alpha_{2 ; *} f_{*} \in L^{2}
$$

as $j \rightarrow \infty$.

Proof Writing

$$
g_{j}=\alpha_{1 ; j} \sum_{-; h_{j}} \alpha_{2 ; j} f_{j}
$$

we see that

$$
\begin{aligned}
\left\|g_{j}\right\|_{L^{2}} & \leq C^{2}\left\|e_{2 \eta} \sum_{-; h_{j}} e_{2 \eta}|f|_{j}\right\|_{L^{2}} \\
& \leq C^{2}\left\|e_{\eta} \sum_{-; h_{j}} e_{2 \eta}|f|_{j}\right\|_{L^{2}} \\
& =C^{2}\left\|\sum_{-; h_{j}} e_{2 \eta}|f|_{j}\right\|_{L_{\eta}^{2}} \\
& \leq C_{3}^{\prime}\left\|f_{j}\right\|_{L_{\eta}^{2}} \\
& \leq C_{3}^{\prime}\left\|f_{j}\right\|_{L^{2}} .
\end{aligned}
$$

In particular, after passing to a subsequence we have the weak convergences $f_{j} \rightarrow f_{*} \in L^{2}$ and $g_{j} \rightarrow g_{*} \in L^{2}$.

Pick any $\zeta \in C_{c}^{\infty}$ and write

$$
\mathcal{I}_{\zeta ; j}=\alpha_{2 ; j} \sum_{+; h_{j}} \alpha_{1 ; j} \zeta-\alpha_{2 ; *} \int_{+} \alpha_{1 ; *} \zeta
$$

which can be expanded as

$$
\begin{aligned}
\mathcal{I}_{\zeta ; j}= & {\left[\alpha_{2 ; j}-\alpha_{2 ; *}\right] \sum_{+; h_{j}} \alpha_{1 ; j} \zeta } \\
& +\alpha_{2 ; *} \sum_{+; h_{j}}\left[\alpha_{1 ; j}-\alpha_{1 ; *}\right] \zeta \\
& +\alpha_{2 ; *}\left[\sum_{+; h_{j}} \alpha_{1 ; *} \zeta-\int_{+} \alpha_{1 ; *} \zeta\right]
\end{aligned}
$$


Using the general observation that $\left\|\sum_{+; h} a b\right\|_{\ell_{h}^{\infty}} \leq\|a\|_{\ell_{h}^{2}}\|b\|_{\ell_{h}^{2}}$, the estimates (4.2) and (4.16) imply that

$$
\begin{aligned}
\left\|\mathcal{I}_{\zeta, j}\right\|_{L^{2}} \leq & 9\left\|\alpha_{2 ; j}-\alpha_{2 ; *}\right\|_{L^{2}}\left\|\alpha_{1 ; j}\right\|_{H^{1}}\|\zeta\|_{H^{1}} \\
& +9\left\|\alpha_{2 ; *}\right\|_{L^{2}}\left\|\alpha_{1 ; j}-\alpha_{1 ; *}\right\|_{H^{1}}\|\zeta\|_{H^{1}} \\
& +\left\|\alpha_{2 ; *}\right\|_{L^{2}}\left|h_{j}\right|\left\|\left[\alpha_{1 ; *} \zeta\right]^{\prime}\right\|_{L^{1}}
\end{aligned}
$$

Observing that

$$
\begin{aligned}
\left\|\left[\alpha_{1 ; *} \zeta\right]^{\prime}\right\|_{L^{1}} & \leq\left\|\alpha_{1 ; *}^{\prime} \zeta\right\|_{L^{1}}+\left\|\alpha_{1 ; *} \zeta^{\prime}\right\|_{L^{1}} \\
& \leq\left\|\alpha_{1 ; *}^{\prime}\right\|_{L^{2}}\|\zeta\|_{L^{2}}+\left\|\alpha_{1 ; *}\right\|_{L^{2}}\left\|\zeta^{\prime}\right\|_{L^{2}} \\
& \leq 2\left\|\alpha_{1 ; *}\right\|_{H^{1}}\|\zeta\|_{H^{1}},
\end{aligned}
$$

we see that $\left\|\mathcal{I}_{\zeta, j}\right\|_{L^{2}} \rightarrow 0$ as $j \rightarrow \infty$. In addition, we see that

$$
\left\|\left(\alpha_{3 ; j}-\alpha_{3 ; *}\right) \zeta\right\|_{L^{2}} \leq\left\|\alpha_{3 ; j}-\alpha_{3 ; *}\right\|_{L^{\infty}}\|\zeta\|_{L^{2}} \leq\left\|\alpha_{3 ; j}-\alpha_{3 ; *}\right\|_{H^{1}}\|\zeta\|_{L^{2}} \rightarrow 0 \text { (4.60) }
$$

as $j \rightarrow \infty$.

We now compute

$$
\begin{aligned}
\left\langle g_{j}, \zeta\right\rangle_{L^{2}} & =\left\langle\alpha_{1 ; j} \sum_{-; h_{j}} \alpha_{2 ; j} f_{j}, \zeta\right\rangle_{L^{2}} \\
& =\left\langle f_{j}, \alpha_{2 ; j} \sum_{+; h_{j}} \alpha_{1 ; j} \zeta\right\rangle_{L^{2}} \\
& =\left\langle f_{j}, \alpha_{2 ; *} \int_{+} \alpha_{1 ; *} \zeta\right\rangle_{L^{2}}+\left\langle f_{j}, \mathcal{I}_{\zeta, j}\right\rangle_{L^{2}},
\end{aligned}
$$

together with

$$
\begin{aligned}
\left\langle\alpha_{3 ; j} f_{j}, \zeta\right\rangle_{L^{2}} & =\left\langle f_{j}, \alpha_{3 ; j} \zeta\right\rangle_{L^{2}} \\
& =\left\langle f_{j}, \alpha_{3 ; *} \zeta\right\rangle_{L^{2}}+\left\langle f_{j},\left(\alpha_{3 ; j}-\alpha_{3 ; *}\right) \zeta\right\rangle_{L^{2}}
\end{aligned}
$$

In particular, the weak convergence $f_{j} \rightarrow f_{*}$ implies that

$$
\begin{aligned}
\left\langle g_{j}, \zeta\right\rangle_{L^{2}} & \rightarrow\left\langle f_{*}, \alpha_{2 ; *} \int_{+} \alpha_{1 ; *} \zeta\right\rangle_{L^{2}} \\
& =\left\langle\alpha_{1 ; *} \int_{-} \alpha_{2 ; *} f_{*}, \zeta\right\rangle_{L^{2}}
\end{aligned}
$$

together with

$$
\begin{aligned}
\left\langle\alpha_{3 ; j} f_{j}, \zeta\right\rangle_{L^{2}} & \rightarrow\left\langle f_{*}, \alpha_{3 ; *} \zeta\right\rangle_{L^{2}} \\
& =\left\langle\alpha_{3 ; *} f_{*}, \zeta\right\rangle_{L^{2}}
\end{aligned}
$$

as $j \rightarrow \infty$. The density of $C_{c}^{\infty}$ in $L^{2}$ now implies the desired weak limits.

\section{Linear Building Blocks}

In this section we are interested in several useful linear operators that act on the sequence spaces $\ell_{h}^{2}$ introduced in Sect. 4 . We use the notation $\partial^{ \pm}, \partial^{0}, \partial^{(2)}$ for the restriction of the discrete derivatives (2.23)-(2.24) to these sequence spaces. In addition, we recall the expressions

$$
\left[T^{ \pm} a\right]_{j h}=a_{(j \pm 1) h}, \quad S^{ \pm} a=\frac{1}{2}\left(a+T^{ \pm} a\right), \quad \gamma_{U}=\sqrt{1-\left(\partial^{0} U\right)^{2}}
$$


that were introduced in [4], together with the higher order norms

$$
\begin{aligned}
& \|V\|_{\ell_{h}^{2 ; 1}}=\|V\|_{\ell_{h}^{2}}+\left\|\partial^{+} V\right\|_{\ell_{h}^{2}}, \\
& \|V\|_{\ell_{h}^{2 ; 2}}=\|V\|_{\ell_{h}^{2}}+\left\|\partial^{+} V\right\|_{\ell_{h}^{2}}+\left\|\partial^{+} \partial^{+} V\right\|_{\ell_{h}^{2}}, \\
& \|V\|_{\ell_{h}^{2 ; 3}}=\|V\|_{\ell_{h}^{2}}+\left\|\partial^{+} V\right\|_{\ell_{h}^{2}}+\left\|\partial^{+} \partial^{+} V\right\|_{\ell_{h}^{2}}+\left\|\partial^{+} \partial^{+} \partial^{+} V\right\|_{\ell_{h}^{2}},
\end{aligned}
$$

and their counterparts

$$
\begin{aligned}
& \|V\|_{\ell_{h}^{\infty ; 1}}=\|V\|_{\ell_{h}^{\infty}}+\left\|\partial^{+} V\right\|_{\ell_{h}^{\infty}}, \\
& \|V\|_{\ell_{h}^{\infty ; 2}}=\|V\|_{\ell_{h}^{\infty}}+\left\|\partial^{+} V\right\|_{\ell_{h}^{\infty}}+\left\|\partial^{+} \partial^{+} V\right\|_{\ell_{h}^{\infty}} .
\end{aligned}
$$

Finally, we recall that $U_{\text {ref } ; *} \in C^{2}(\mathbb{R},[0,1])$ stands for a reference function that satisfies the properties

$$
U_{\text {ref } ; *}((-\infty,-2])=0, \quad U_{\text {ref; } *}([2, \infty))=1, \quad 0 \leq U_{\text {ref } ; *}^{\prime}<1, \quad\left|U_{\text {ref;* }}^{\prime \prime}\right|<1 .
$$

For any $\kappa>0$, we subsequently write

$$
U_{\text {ref } ; \kappa}(\tau)=U_{\text {ref;* }}(\kappa \tau)
$$

and introduce an open subset

$$
\mathcal{V}_{h ; \kappa}=\left\{V \in \ell_{h}^{2}:\|V\|_{\ell_{h}^{2 ; 2}}+\|V\|_{\ell_{h}^{\infty}}+\left\|\partial^{+} \partial^{+} V\right\|_{\ell_{h}^{\infty}}<\frac{1}{2} \kappa^{-1} \text { and }\left\|\partial^{+} V\right\|_{\infty}<1-2 \kappa\right\} .
$$

This allows us to recall the affine subset [4]

$$
\Omega_{h ; \kappa}=U_{\text {ref } ; \kappa}(h \mathbb{Z})+\mathcal{V}_{h ; \kappa} \subset \ell_{h}^{\infty}
$$

that plays an important role here and in the sequel paper [5], as it captures the admissable states of the waves that we are interested in. We remind the reader that each $U \in \Omega_{h ; \kappa}$ satisfies $\left\|\partial^{+} U\right\|_{\infty} \leq 1-\kappa$ and that the norms $\left\|\partial^{+} U\right\|_{\ell_{h}^{2,1}},\|U\|_{\ell_{h}^{\infty ; 2}}$ and $\|g(U)\|_{\ell_{h}^{2}}$ are all bounded uniformly in $h>0$.

The linear operators that we investigate are given by

$$
\begin{aligned}
& M_{U ; A}[V]=4 \gamma_{U}^{-4} \partial^{0} U\left[\partial^{(2)} U\right] \partial^{0} V, \quad M_{U ; C}[V]=\gamma_{U}^{2} g^{\prime}(U) V, \\
& M_{U ; B}[V]=\gamma_{U}^{-2} \partial^{(2)} V, \quad M_{U ; D}[V]=-c_{*} \gamma_{U}^{-1} \partial^{0} V .
\end{aligned}
$$

Here we have $V \in \ell_{h}^{2}$, while $U$ is taken from $\Omega_{h ; \kappa}$. For convenience, we introduce the combination

$$
M_{U}[V]=M_{U ; A}[V]+M_{U ; B}[V]+M_{U ; C}[V]+M_{U ; D}[V]
$$

together with the notation

$$
L_{U}[V]=c_{*} \partial^{0} V+M_{U}[V]+\partial^{0} U \sum_{-; h} \gamma_{U}^{-2}\left[\partial^{(2)} U\right] M_{U}[V]
$$

Picking any $v \in H^{1}$ and recalling the discrete evaluation operator

$$
\left[\operatorname{ev}_{\vartheta} f\right]_{j h}=f(\vartheta+j h)
$$

we note that our construction implies that the identities

$$
\operatorname{ev}_{\vartheta} M_{h}[v]=M_{\mathrm{ev}_{\vartheta} \Psi_{*}}\left[\operatorname{ev}_{\vartheta} v\right], \quad \operatorname{ev}_{\vartheta}\left[c_{*} v^{\prime}+\mathcal{L}_{h}[v]\right]=L_{\mathrm{ev}_{\vartheta} \Psi_{*}}\left[\operatorname{ev}_{\vartheta} v\right]
$$


hold for all $\vartheta \in[0, h]$. We remark that the right-hand sides above are continuous in $\ell_{h}^{2}$ as a function of $\vartheta$ as a consequence of (4.2) and the continuity of the translation operator on $H^{1}$. We recall from item (iii) of [4, Lem. A.4] that if

$$
\left\|\operatorname{ev}_{\vartheta} f\right\|_{\ell_{h}^{2}} \leq\|\operatorname{ev} \vartheta v\|_{\ell_{h}^{2 ; 2}}
$$

holds for all $\vartheta \in(0, h)$ and some $v \in H^{1}$, then in fact $f \in L^{2}$ with

$$
\|f\|_{L^{2}} \leq\|v\|_{H^{1}}+\left\|\partial_{h}^{+} \partial_{h}^{+} v\right\|_{L^{2}} .
$$

We are specifically interested in the differences $\partial^{+} M_{U}[V]$ and $\partial^{+} L_{U}[V]$, as they will help us to apply a discrete derivative to the equation $\mathcal{L}_{h} v=f$ and its nonlinear counterpart that will appear in [5]. To this end, we introduce the approximate differences

$$
\begin{aligned}
M_{U ; A ; \text { apx }}^{+}[V]= & 4\left(4 \gamma_{U}^{-6}-3 \gamma_{U}^{-4}\right)\left[\partial^{(2)} U\right]^{2} \partial^{0} V+4 \gamma_{U}^{-4} \partial^{0} U\left[\partial^{+} \partial^{(2)} U\right] \partial^{0} V \\
& +4 \gamma_{U}^{-4} \partial^{0} U\left[\partial^{(2)} U\right] \partial^{(2)} V, \\
M_{U ; B ; \text { apx }}^{+}[V]= & 2 \gamma_{U}^{-4} \partial^{0} U\left[\partial^{(2)} U\right] \partial^{(2)} V+\gamma_{U}^{-2} \partial^{+} \partial^{(2)} V, \\
M_{U ; C ; \text { apx }}^{+}[V]= & -2 \partial^{0} U\left[\partial^{(2)} U\right] g^{\prime}(U) V+\gamma_{U}^{2} g^{\prime \prime}(U)\left[\partial^{0} U\right] V+\gamma_{U}^{2} g^{\prime}(U) \partial^{0} V, \\
M_{U ; D ; \text { apx }}^{+}[V]= & -c_{*} \gamma_{U}^{-3} \partial^{0} U\left[\partial^{(2)} U\right] \partial^{0} V-c_{*} \gamma_{U}^{-1} \partial^{(2)} V
\end{aligned}
$$

and write

$$
M_{U ; \mathrm{apx}}^{+}[V]=M_{U ; A ; \mathrm{apx}}^{+}[V]+M_{U ; B ; \mathrm{apx}}^{+}[V]+M_{U ; C ; \mathrm{apx}}^{+}[V]+M_{U ; D ; \mathrm{apx}}^{+}[V] .
$$

Proposition 5.1 Assume that $(\mathrm{Hg})$ is satisfied and fix $\kappa>0$. There exists $K>0$ so that for any $h>0, U \in \Omega_{h ; \kappa}$ and $V \in \ell_{h}^{2}$ we have the a-priori bounds

$$
\begin{array}{ll}
\left\|M_{U}[V]\right\|_{\ell_{h}^{2}} & \leq K\|V\|_{\ell_{h}^{2 ; 2}}, \\
\left\|\partial^{+} M_{U}[V]\right\|_{\ell_{h}^{2}} & \leq K\|V\|_{\ell_{h}^{2 ; 3}}+K\left\|\partial^{+} \partial^{+} \partial^{+} U\right\|_{\ell_{h}^{\infty}}\left\|\partial^{+} V\right\|_{\ell_{h}^{2}}, \\
\left\|\partial^{+} M_{U}[V]-M_{U}\left[\partial^{+} V\right]\right\|_{\ell_{h}^{2}} & \leq K\|V\|_{\ell_{h}^{2 ; 2}}+K\left\|\partial^{+} \partial^{+} \partial^{+} U\right\|_{\ell_{h}^{\infty}}\left\|\partial^{+} V\right\|_{\ell_{h}^{2}},
\end{array}
$$

together with the estimate

$$
\left\|\partial^{+} M_{U}[V]-M_{U ; \mathrm{apx}}^{+}[V]\right\|_{\ell_{h}^{2}} \leq K h\|V\|_{\ell_{h}^{2 ; 3}}+K h\left\|\partial^{+} \partial^{+} \partial^{+} U\right\|_{\ell_{h}^{\infty}}\|V\|_{\ell_{h}^{2 ; 2}} .
$$

In addition, for any $h>0$, any pair $\left(U^{(1)}, U^{(2)}\right) \in \Omega_{h ; \kappa}^{2}$ and any $V \in \ell_{h}^{2}$, we have the Lipschitz bound

$$
\left\|M_{U^{(2)}}[V]-M_{U^{(1)}}[V]\right\|_{\ell_{h}^{2}} \leq K\left\|U^{(2)}-U^{(1)}\right\|_{\ell_{h}^{2 ; 2}}\|V\|_{\ell_{h}^{\infty ; 1}}+K\left\|U^{(2)}-U^{(1)}\right\|_{\ell_{h}^{\infty ; 1}}\|V\|_{\ell_{h}^{2 ; 2}} .
$$

Corollary 5.2 Assume that $(\mathrm{Hg})$ is satisfied and pick $0<\kappa<\frac{1}{12}$. Then there exists a constant $K>0$ so that for any $h>0, U \in \Omega_{h ; \kappa}$ and $V \in \ell_{h}^{2}$ we have the estimate

$$
\left\|\partial^{+}\left[L_{U}[V]\right]-L_{U}\left[\partial^{+} V\right]\right\|_{\ell_{h}^{2}} \leq K\left[1+\left\|\partial^{+} \partial^{+} \partial^{+} U\right\|_{\ell_{h}^{\infty}}+\left\|\partial^{+} \partial^{+} \partial^{+} U\right\|_{\ell_{h}^{2}}\right]\|V\|_{\ell_{h}^{2 ; 2}} .
$$


Proof Systematically applying the product rule $\partial^{+}[a b]=\partial^{+} a T^{+} b+a \partial^{+} b$ and the identity $\partial^{+} \partial^{0}=S^{+} \partial^{(2)}$ described in $[4, \S 3.1]$, we compute

$$
\begin{aligned}
\partial^{+}\left[L_{U}[V]\right]= & c_{*} S^{+}\left[\partial^{(2)} V\right]+\partial^{+}\left[M_{U}[V]\right] \\
& +S^{+}\left[\partial^{(2)} U\right] T^{+} \sum_{-; h} \gamma_{U}^{-2}\left[\partial^{(2)} U\right] M_{U}[V] \\
& +\partial^{0} U \sum_{-; h} \partial^{+}\left[\gamma_{U}^{-2}\right] T^{+}\left[\partial^{(2)} U M_{U}[V]\right] \\
& +\partial^{0} U \sum_{-; h} \gamma_{U}^{-2} \partial^{+}\left[\partial^{(2)} U\right] T^{+}\left[M_{U}[V]\right] \\
& +\partial^{0} U \sum_{-; h} \gamma_{U}^{-2}\left[\partial^{(2)} U\right] \partial^{+}\left[M_{U}[V]\right] .
\end{aligned}
$$

On the other hand, a direct substitution yields

$$
L_{U}\left[\partial^{+} V\right]=c_{*} S^{+}\left[\partial^{(2)} V\right]+M_{U}\left[\partial^{+} V\right]+\partial^{0} U \sum_{-; h} \gamma_{U}^{-2}\left[\partial^{(2)} U\right] M_{U}\left[\partial^{+} V\right] .
$$

Comparing these two expressions, we obtain the bound

$$
\begin{aligned}
\left\|\partial^{+}\left[L_{U}[V]\right]-L_{U}\left[\partial^{+} V\right]\right\|_{\ell_{h}^{2}} \leq & C_{1}^{\prime}\left\|\partial^{+}\left[M_{U}[V]\right]-M_{U}\left[\partial^{+} V\right]\right\|_{\ell_{h}^{2}}+C_{1}^{\prime}\left\|M_{U}[V]\right\|_{\ell_{h}^{2}} \\
& +C_{1}^{\prime}\left\|\partial^{+} \partial^{+} \partial^{+} U\right\|_{\ell_{h}^{2}}\left\|M_{U}[V]\right\|_{\ell_{h}^{2}} .
\end{aligned}
$$

The desired estimate now follows from (5.17).

Corollary 5.3 Assume that $(\mathrm{Hg})$ is satisfied and pick $0<\kappa<\frac{1}{12}$. There exists a constant $K>0$ so that the estimate

$$
\begin{aligned}
\left\|L_{U^{(2)}}[V]-L_{U^{(1)}}[V]\right\|_{\ell_{h}^{2}} \leq & K\left\|U^{(2)}-U^{(1)}\right\|_{\ell_{h}^{2 ; 2}}\|V\|_{\ell_{h}^{\infty ; 1}}+K\left\|U^{(2)}-U^{(1)}\right\|_{\ell_{h}^{\infty ; 1}}\|V\|_{\ell_{h}^{2 ; 2}} \\
& +K\left\|U^{(2)}-U^{(1)}\right\|_{\ell_{h}^{2 ; 2}}\|V\|_{\ell_{h}^{2 ; 2}}
\end{aligned}
$$

holds for all $h>0$, all $V \in \ell_{h}^{2}$ and all pairs $\left(U^{(1)}, U^{(2)}\right) \in \Omega_{h ; \kappa}^{2}$.

Proof We compute

$$
\begin{aligned}
\left\|L_{U^{(2)}}[V]-L_{U^{(1)}}[V]\right\|_{\ell_{h}^{2}} \leq & \left\|M_{U^{(2)}}[V]-M_{U^{(1)}}[V]\right\|_{\ell_{h}^{2}}+C_{1}^{\prime}\left\|\partial^{0} U^{(2)}-\partial^{0} U^{(1)}\right\|_{\ell_{h}^{2}}\left\|M_{U_{2}}[V]\right\|_{\ell_{h}^{2}} \\
& +C_{1}^{\prime}\left\|\gamma_{U^{(2)}} \partial^{(2)} U^{(2)}-\gamma_{U^{(1)}} \partial^{(2)} U^{(1)}\right\|_{\ell_{h}^{2}}\left\|M_{U_{2}}[V]\right\|_{\ell_{h}^{2}} \\
& +C_{1}^{\prime}\left\|M_{U^{(2)}}[V]-M_{U^{(1)}}[V]\right\|_{\ell_{h}^{2}} .
\end{aligned}
$$

Exploiting the a-priori bound (5.17) together with the Lipschitz bounds (A.7) and (5.19), this yields the desired estimate.

Corollary 5.4 Assume that $(\mathrm{Hg})$ is satisfied. Then there exists a constant $K>0$ so that the estimate

$$
\left\|\partial_{h}^{+} \mathcal{L}_{h}[v]-\mathcal{L}_{h}\left[\partial_{h}^{+} v\right]\right\|_{L^{2}} \leq K\left[\|v\|_{H^{1}}+\left\|\partial_{h}^{+} \partial_{h}^{+} v\right\|_{L^{2}}\right]
$$

holds for all $h>0$ and all $v \in H^{1}$.

Proof The result follows from Corollary 5.2 and the bound (5.14).

In the sequel we will also encounter the expressions

$$
\tilde{M}_{U ; \#}[V]=\gamma_{U}^{-2} M_{U ; \# ; \text { apx }}^{+}[V]+2 \gamma_{U}^{-4} \partial^{0} U\left[\partial^{(2)} U\right] M_{U ; \#}[V]
$$


for \# $\in\{A, B, C, D\}$, together with

$$
\widetilde{M}_{U ; E}[V]=4 \gamma_{U}^{-6} \partial^{0} U\left[\partial^{+} \partial^{(2)} U\right] \partial^{0} V+\gamma_{U}^{-4} \partial^{+} \partial^{(2)} V .
$$

The relevant combinations are evaluated explicitly in the final main result of this section.

Proposition 5.5 For any $\kappa>0, h>0, U \in \Omega_{h ; \kappa}$ and $V \in \ell_{h}^{2}$, we have the identities

$$
\begin{aligned}
\tilde{M}_{U ; A}[V]+\tilde{M}_{U ; B}[V]+\tilde{M}_{U ; C}[V]= & 4\left[6 \gamma_{U}^{-8}-5 \gamma_{U}^{-6}\right]\left[\partial^{(2)} U\right]^{2} \partial^{0} V \\
& +8 \gamma_{U}^{-6} \partial^{0} U\left[\partial^{(2)} U\right] \partial^{(2)} V \\
& +g^{\prime \prime}(U)\left[\partial^{0} U\right] V+g^{\prime}(U) \partial^{0} V+\tilde{M}_{U ; E}[V], \\
\tilde{M}_{U ; D}[V]= & -3 c_{*} \gamma_{U}^{-5} \partial^{0} U\left[\partial^{(2)} U\right] \partial^{0} V-c_{*} \gamma_{U}^{-3} \partial^{(2)} V .
\end{aligned}
$$

\subsection{Proof of Propositions 5.1 and 5.5}

We first set out to establish Proposition 5.1. We will treat each of the four components separately, using the estimates (A.8) to approximate the $\partial^{+}\left[\gamma_{U}^{-k}\right]$ terms.

Lemma 5.6 Fix $\kappa>0$. There exist $K>0$ so that for any $h>0, U \in \Omega_{h ; \kappa}$ and $V \in \ell_{h}^{2}$ we have the bound

$$
\left\|\partial^{+} M_{U ; A}[V]-M_{U ; A}\left[\partial^{+} V\right]\right\|_{\ell_{h}^{2}} \leq K\left\|\partial^{+} V\right\|_{\ell_{h}^{2}}+K\left\|\partial^{+} \partial^{+} \partial^{+} U\right\|_{\ell_{h}^{\infty}}\left\|\partial^{+} V\right\|_{\ell_{h}^{2}},
$$

together with the estimate

$$
\begin{aligned}
\left\|\partial^{+} M_{U ; A}[V]-M_{U ; A ; a p x}^{+}[V]\right\|_{\ell_{h}^{2}} \leq & K h\left[\left\|\partial^{+} V\right\|_{\ell_{h}^{2}}+\left\|\partial^{+} \partial^{+} V\right\|_{\ell_{h}^{2}}+\left\|\partial^{+} \partial^{+} \partial^{+} V\right\|_{\ell_{h}^{2}}\right] \\
& +K h\left\|\partial^{+} \partial^{+} \partial^{+} U\right\|_{\ell_{h}^{\infty}}\left\|\partial^{+} V\right\|_{\ell_{h}^{2}} .
\end{aligned}
$$

Proof We compute

$$
\begin{aligned}
\partial^{+} M_{U ; A}[V]= & 4 \partial^{+}\left[\gamma_{U}^{-4}\right] T^{+}\left[\partial^{0} U\left[\partial^{(2)} U\right] \partial^{0} V\right] \\
& +4 \gamma_{U}^{-4} S^{+}\left[\partial^{(2)} U\right] T^{+}\left[\partial^{(2)} U\right] T^{+} \partial^{0} V \\
& +4 \gamma_{U}^{-4} \partial^{0} U \partial^{+}\left[\partial^{(2)} U\right] T^{+} \partial^{0} V \\
& +4 \gamma_{U}^{-4} \partial^{0} U\left[\partial^{(2)} U\right] S^{+}\left[\partial^{(2)} V\right],
\end{aligned}
$$

together with

$$
M_{U ; A}\left[\partial^{+} V\right]=4 \gamma_{U}^{-4} \partial^{0} U\left[\partial^{(2)} U\right] S^{+}\left[\partial^{(2)} V\right] .
$$

The estimate (5.30) now follows directly from inspection.

Upon making the replacements

$$
\partial^{+}\left[\gamma_{U}^{-4}\right] \mapsto 4 \gamma_{U}^{-6} \partial^{0} U\left[\partial^{(2)} U\right], \quad T^{+} \mapsto I, \quad S^{+} \mapsto I,
$$

we readily see that $\partial^{+}\left[M_{U ; A}[V]\right]$ agrees with $M_{U ; A ; \text { apx }}^{+}[V]$. In particular, applying these replacements to each of the four terms in (5.32) separately, we may write

$$
\partial^{+}\left[M_{U ; A}[V]\right]-M_{U ; A ; \mathrm{apx}}^{+}[V]=\mathcal{J}_{a}+\mathcal{J}_{b}+\mathcal{J}_{c}+\mathcal{J}_{d},
$$


in which

$$
\begin{aligned}
\mathcal{J}_{a}= & 4\left[\partial^{+}\left[\gamma_{U}^{-4}\right]-4 \gamma_{U}^{-6} \partial^{0} U S^{+}\left[\partial^{(2)} U\right]\right] T^{+}\left[\partial^{0} U\left[\partial^{(2)} U\right] \partial^{0} V\right] \\
& +8 h \gamma_{U}^{-6} \partial^{0} U \partial^{+}\left[\partial^{(2)} U\right] T^{+}\left[\partial^{0} U\left[\partial^{(2)} U\right] \partial^{0} V\right] \\
& +16 h \gamma_{U}^{-6} \partial^{0} U\left[\partial^{(2)} U\right] S^{+}\left[\partial^{(2)} U\right] T^{+}\left[\partial^{(2)} U\right] T^{+}\left[\partial^{0} V\right] \\
& +16 h \gamma_{U}^{-6} \partial^{0} U\left[\partial^{(2)} U\right] \partial^{0} U \partial^{+}\left[\partial^{(2)} U\right] T^{+}\left[\partial^{0} V\right] \\
& +16 h \gamma_{U}^{-6} \partial^{0} U\left[\partial^{(2)} U\right] \partial^{0} U\left[\partial^{(2)} U\right] S^{+}\left[\partial^{(2)} V\right],
\end{aligned}
$$

together with

$$
\begin{aligned}
\mathcal{J}_{b}= & 2 h \gamma_{U}^{-4} \partial^{+}\left[\partial^{(2)} U\right] T^{+}\left[\partial^{(2)} U\right] T^{+}\left[\partial^{0} V\right] \\
& +4 h \gamma_{U}^{-4}\left[\partial^{(2)} U\right] \partial^{+}\left[\partial^{(2)} U\right] T^{+}\left[\partial^{0} V\right] \\
& +4 h \gamma_{U}^{-4}\left[\partial^{(2)} U\right]\left[\partial^{(2)} U\right] S^{+}\left[\partial^{(2)} V\right]
\end{aligned}
$$

and finally

$$
\begin{aligned}
& \mathcal{J}_{c}=4 h \gamma_{U}^{-4} \partial^{0} U \partial^{+}\left[\partial^{(2)} U\right] S^{+}\left[\partial^{(2)} V\right], \\
& \mathcal{J}_{d}=2 h \gamma_{U}^{-4} \partial^{0} U\left[\partial^{(2)} U\right] \partial^{+}\left[\partial^{(2)} V\right] .
\end{aligned}
$$

The desired estimate (5.31) follows from (A.8) and inspection of the above identities.

Lemma 5.7 Fix $\kappa>0$. There exist $K>0$ so that for any $h>0, U \in \Omega_{h ; \kappa}$ and $V \in \ell_{h}^{2}$ we have the bound

$$
\left\|\partial^{+} M_{U ; B}[V]-M_{U ; B}\left[\partial^{+} V\right]\right\|_{\ell_{h}^{2}} \leq K\left\|\partial^{+} \partial^{+} V\right\|_{\ell_{h}^{2}},
$$

together with the estimate

$$
\begin{aligned}
\left\|\partial^{+} M_{U ; B}[V]-M_{U ; B ; \text { apx }}^{+}[V]\right\|_{\ell_{h}^{2}} \leq & K h\left[\left\|\partial^{+} \partial^{+} V\right\|_{\ell_{h}^{2}}+\left\|\partial^{+} \partial^{+} \partial^{+} V\right\|_{\ell_{h}^{2}}\right] \\
& +K h\left\|\partial^{+} \partial^{+} \partial^{+} U\right\|_{\ell_{h}^{\infty}}\left\|\partial^{+} \partial^{+} V\right\|_{\ell_{h}^{2}} .
\end{aligned}
$$

Proof We compute

$$
\partial^{+} M_{U ; B}[V]=\partial^{+}\left[\gamma_{U}^{-2}\right] T^{+}\left[\partial^{(2)} V\right]+\gamma_{U}^{-2} \partial^{+} \partial^{(2)} V,
$$

together with

$$
M_{U ; B}\left[\partial^{+} V\right]=\gamma_{U}^{-2} \partial^{+} \partial^{(2)} V .
$$

The estimate (5.39) now follows directly from inspection.

Upon making the replacements

$$
\partial^{+}\left[\gamma_{U}^{-2}\right] \mapsto 2 \gamma_{U}^{-4} \partial^{0} U\left[\partial^{(2)} U\right], \quad T^{+} \mapsto I,
$$

we readily see that $\partial^{+}\left[M_{U ; B}[V]\right]$ agrees with $M_{U ; B ; \text { apx }}^{+}[V]$. In particular, we may write

$$
\partial^{+}\left[M_{U ; B}[V]\right]-M_{U ; B ; \mathrm{apx}}^{+}[V]=\mathcal{J}_{a}
$$

in which

$$
\begin{aligned}
\mathcal{J}_{a}= & {\left[\partial^{+}\left[\gamma_{U}^{-2}\right]-2 \gamma_{U}^{-4} \partial^{0} U S^{+}\left[\partial^{(2)} U\right]\right] T^{+}\left[\partial^{(2)} V\right] } \\
& +h \gamma_{U}^{-4} \partial^{0} U \partial^{+}\left[\partial^{(2)} U\right] T^{+}\left[\partial^{(2)} V\right] \\
& +2 h \gamma_{U}^{-4} \partial^{0} U\left[\partial^{(2)} U\right] \partial^{+}\left[\partial^{(2)} V\right] .
\end{aligned}
$$

The desired estimate (5.40) follows from (A.8) and inspection of the above identity. 
Lemma 5.8 Assume that $(\mathrm{Hg})$ is satisfied and fix $\kappa>0$. There exist $K>0$ so that for any $h>0, U \in \Omega_{h ; \kappa}$ and $V \in \ell_{h}^{2}$ we have the bound

$$
\left\|\partial^{+} M_{U ; C}[V]-M_{U ; C}\left[\partial^{+} V\right]\right\|_{\ell_{h}^{2}} \leq K\|V\|_{\ell_{h}^{2}},
$$

together with the estimate

$$
\begin{aligned}
\left\|\partial^{+} M_{U ; C}[V]-M_{U ; C ; \text { apx }}^{+}[V]\right\|_{\ell_{h}^{2}} \leq & K h\left[\|V\|_{\ell_{h}^{2}}+\left\|\partial^{+} V\right\|_{\ell_{h}^{2}}+\left\|\partial^{+} \partial^{+} V\right\|_{\ell_{h}^{2}}\right] \\
& +K h\left\|\partial^{+} \partial^{+} \partial^{+} U\right\|_{\ell_{h}^{\infty}}\|V\|_{\ell_{h}^{2}} .
\end{aligned}
$$

Proof We compute

$$
\partial^{+} M_{U ; C}[V]=\partial^{+}\left[\gamma_{U}^{2}\right] T^{+}\left[g^{\prime}(U) V\right]+\gamma_{U}^{2} \partial^{+}\left[g^{\prime}(U)\right] T^{+} V+\gamma_{U}^{2} g^{\prime}(U) \partial^{+} V,
$$

together with

$$
M_{U ; C}\left[\partial^{+} V\right]=\gamma_{U}^{2} g^{\prime}(U) \partial^{+} V .
$$

The estimate (5.46) now follows directly from inspection.

Upon making the replacements

$$
\partial^{+}\left[\gamma_{U}^{-2}\right] \mapsto 2 \gamma_{U}^{-4} \partial^{0} U\left[\partial^{(2)} U\right], \quad T^{+} \mapsto I, \quad \partial^{+}\left[g^{\prime}(U)\right] \mapsto g^{\prime \prime}(U) \partial^{0} U
$$

we readily see that $\partial^{+}\left[M_{U ; C}[V]\right]$ agrees with $M_{U ; C ; \text { apx }}^{+}[V]$. In particular, applying these replacements to each of the three terms in (5.48) separately, we may write

$$
\partial^{+}\left[M_{U ; C}[V]\right]-M_{U ; C ; \mathrm{apx}}^{+}[V]=\mathcal{J}_{a}+\mathcal{J}_{b}+\mathcal{J}_{c},
$$

in which

$$
\begin{aligned}
\mathcal{J}_{a}= & {\left[\partial^{+}\left[\gamma_{U}^{2}\right]+2 \partial^{0} U S^{+}\left[\partial^{(2)} U\right]\right] T^{+}\left[g^{\prime}(U) V\right] } \\
& -h \partial^{0} U \partial^{+}\left[\partial^{(2)} U\right] T^{+}\left[g^{\prime}(U) V\right] \\
& -2 h \partial^{0} U\left[\partial^{(2)} U\right] \partial^{+}\left[g^{\prime}(U)\right] T^{+} V \\
& -2 h \partial^{0} U\left[\partial^{(2)} U\right] g^{\prime}(U) \partial^{+} V,
\end{aligned}
$$

together with

$$
\begin{aligned}
\mathcal{J}_{b}= & \gamma_{U}^{2}\left[\partial^{+} g^{\prime}(U)-g^{\prime \prime}(U) \partial^{0} U\right] T^{+} V \\
& +\gamma_{U}^{2} g^{\prime \prime}(U)\left[\partial^{+} U-\partial^{0} U\right] T^{+} V \\
& +h \gamma_{U}^{2} g^{\prime \prime}(U)\left[\partial^{0} U\right] \partial^{+} V
\end{aligned}
$$

and finally

$$
\begin{aligned}
\mathcal{J}_{c} & =\gamma_{U}^{2} g^{\prime}(U)\left[\partial^{+} V-\partial^{0} V\right] \\
& =\frac{1}{2} h \gamma_{U}^{2} g^{\prime}(U) \partial^{(2)} V .
\end{aligned}
$$

In order to estimate $\left\|\mathcal{J}_{b}\right\|_{\ell_{h}^{2}}$, we recall that $\partial^{+} U-\partial^{0} U=\frac{1}{2} h \partial^{(2)} U$ and compute

$$
\begin{aligned}
\left|\partial^{+} g^{\prime}(U)-g^{\prime \prime}(U) \partial^{+} U\right| & =h^{-1}\left|g^{\prime}\left(U+h \partial^{+} U\right)-g^{\prime}(U)-g^{\prime \prime}(U) h \partial^{+} U\right| \\
& \leq \frac{1}{2}\left[\sup _{|u| \leq \kappa^{-1}}\left|g^{\prime \prime \prime}(u)\right|\right] h^{-1}\left|h \partial^{+} U\right|^{2} \\
& =\frac{1}{2} h\left[\sup _{|u| \leq \kappa^{-1}}\left|g^{\prime \prime \prime}(u)\right|\right]\left|\partial^{+} U\right|^{2} .
\end{aligned}
$$

The desired estimate (5.47) now follows from (A.8) and inspection of the above identities. 
Lemma 5.9 Fix $\kappa>0$. There exist $K>0$ so that for any $h>0, U \in \Omega_{h ; \kappa}$ and $V \in \ell_{h}^{2}$ we have the bound

$$
\left\|\partial^{+} M_{U ; D}[V]-M_{U ; D}\left[\partial^{+} V\right]\right\|_{\ell_{h}^{2}} \leq K\left\|\partial^{+} V\right\|_{\ell_{h}^{2}},
$$

together with the estimate

$$
\begin{aligned}
\left\|\partial^{+} M_{U ; D}[V]-M_{U ; D ; \text { ap }}^{+}[V]\right\|_{\ell_{h}^{2}} \leq & K h\left[\left\|\partial^{+} V\right\|_{\ell_{h}^{2}}+\left\|\partial^{+} \partial^{+} V\right\|_{\ell_{h}^{2}}+\left\|\partial^{+} \partial^{+} \partial^{+} V\right\|_{\ell_{h}^{2}}\right] \\
& +K h\left\|\partial^{+} \partial^{+} \partial^{+} U\right\|_{\ell_{h}^{\infty}}\left\|\partial^{+} V\right\|_{\ell_{h}^{2}}
\end{aligned}
$$

Proof We compute

$$
\partial^{+} M_{U ; D}[V]=-c_{*} \partial^{+}\left[\gamma_{U}^{-1}\right] T^{+} \partial^{0} V-c_{*} \gamma_{U}^{-1} S^{+}\left[\partial^{(2)} V\right],
$$

together with

$$
M_{U ; D}\left[\partial^{+} V\right]=-c_{*} \gamma_{U}^{-1} S^{+}\left[\partial^{(2)} V\right] .
$$

The estimate (5.56) now follows directly from inspection.

Upon making the replacements

$$
\partial^{+}\left[\gamma_{U}^{-1}\right] \mapsto \gamma_{U}^{-3} \partial^{0} U\left[\partial^{(2)} U\right], \quad T^{+} \mapsto I, \quad S^{+} \mapsto I
$$

we readily see that $\partial^{+}\left[M_{U ; D}[V]\right]$ agrees with $M_{U ; D ; \text { apx }}^{+}[V]$. In particular, applying these replacements to each of the two terms in (5.48) separately, we see that

$$
\partial^{+}\left[M_{U ; D}[V]\right]-M_{U ; D ; \text { apx }}^{+}[V]=\mathcal{J}_{a}+\mathcal{J}_{b}
$$

in which

$$
\begin{aligned}
\mathcal{J}_{a}= & -c_{*}\left[\partial^{+}\left[\gamma_{U}^{-1}\right]-\gamma_{U}^{-3} \partial^{0} U S^{+}\left[\partial^{(2)} U\right]\right] T^{+}\left[\partial^{0} V\right] \\
& -\frac{1}{2} c_{*} h \gamma_{U}^{-3} \partial^{0} U \partial^{+}\left[\partial^{(2)} U\right] T^{+}\left[\partial^{0} V\right] \\
& -c_{*} h \gamma_{U}^{-3} \partial^{0} U\left[\partial^{(2)} U\right] S^{+}\left[\partial^{(2)} V\right],
\end{aligned}
$$

together with

$$
\mathcal{J}_{b}=-\frac{1}{2} c_{*} h \partial^{+}\left[\partial^{(2)} V\right] .
$$

The desired estimate (5.57) now follows from (A.8) and inspection of the above identities.

Proof of Proposition 5.1 The bound for $\left\|M_{U}[V]\right\|_{\ell_{h}^{2}}$ and the Lipschitz bound (5.19) follow directly by inspecting the definitions (5.8). The remaining bounds follow from Lemma's 5.6-5.9.

Proof of Proposition 5.5 Direct computations yield

$$
\begin{aligned}
\tilde{M}_{U ; A}[V]= & 4\left(4 \gamma_{U}^{-8}-3 \gamma_{U}^{-6}\right)\left[\partial^{(2)} U\right]^{2} \partial^{0} V+4 \gamma_{U}^{-6} \partial^{0} U\left[\partial^{+} \partial^{(2)} U\right] \partial^{0} V \\
& +4 \gamma_{U}^{-6} \partial^{0} U\left[\partial^{(2)} U\right] \partial^{(2)} V+8 \gamma_{U}^{-8} \partial^{0} U\left[\partial^{(2)} U\right] \partial^{0} U\left[\partial^{(2)} U\right] \partial^{0} V \\
= & 4\left(6 \gamma_{U}^{-8}-5 \gamma_{U}^{-6}\right)\left[\partial^{(2)} U\right]^{2} \partial^{0} V \\
& +4 \gamma_{U}^{-6} \partial^{0} U\left[\partial^{+} \partial^{(2)} U\right] \partial^{0} V+4 \gamma_{U}^{-6} \partial^{0} U\left[\partial^{(2)} U\right] \partial^{(2)} V,
\end{aligned}
$$

together with

$$
\begin{aligned}
\tilde{M}_{U ; B}[V] & =2 \gamma_{U}^{-6} \partial^{0} U\left[\partial^{(2)} U\right] \partial^{(2)} V+\gamma_{U}^{-4} \partial^{+} \partial^{(2)} V+2 \gamma_{U}^{-6} \partial^{0} U\left[\partial^{(2)} U\right] \partial^{(2)} V \\
& =4 \gamma_{U}^{-6} \partial^{0} U\left[\partial^{(2)} U\right] \partial^{(2)} V+\gamma_{U}^{-4} \partial^{+} \partial^{(2)} V
\end{aligned}
$$


and finally

$$
\begin{aligned}
\widetilde{M}_{U ; C}[V]= & -2 \gamma_{U}^{-2} \partial^{0} U\left[\partial^{(2)} U\right] g^{\prime}(U) V+g^{\prime \prime}(U)\left[\partial^{0} U\right] V+g^{\prime}(U) \partial^{0} V \\
& +2 \gamma_{U}^{-2} \partial^{0} U\left[\partial^{(2)} U\right] g^{\prime}(U) V \\
= & g^{\prime \prime}(U)\left[\partial^{0} U\right] V+g^{\prime}(U) \partial^{0} V .
\end{aligned}
$$

The first identity follows directly from these expressions. To obtain the second identity we compute

$$
\begin{aligned}
\tilde{M}_{U ; D}[V]= & -c_{*} \gamma_{U}^{-5} \partial^{0} U\left[\partial^{(2)} U\right] \partial^{0} V-c_{*} \gamma_{U}^{-3} \partial^{(2)} V \\
& -2 c_{*} \gamma_{U}^{-5} \partial^{0} U\left[\partial^{(2)} U\right] \partial^{0} V \\
= & -3 c_{*} \gamma_{U}^{-5} \partial^{0} U\left[\partial^{(2)} U\right] \partial^{0} V-c_{*} \gamma_{U}^{-3} \partial^{(2)} V .
\end{aligned}
$$

\section{The Full Linear Operator}

In this section we set out to construct solutions to the inhomogeneous problem $\mathcal{L}_{h} v=f$ and establish Theorem 2.3. Taking $v \in H^{1}$ and $f \in L^{2}$, we first recall (5.12) and emphasize that this problem should be interpreted as the statement that

$$
L_{\mathrm{ev}_{\vartheta} \Psi_{*}}\left[\operatorname{ev}_{\vartheta} v\right]=\operatorname{ev}_{\vartheta}\left[c_{*} v^{\prime}+f\right]
$$

holds for almost all $\vartheta \in[0, h]$. Throughout the sequel we simply use the notation (2.28) and keep this interpretation in mind.

Our strategy is to apply the spirit of the ideas in [1] to our present more convoluted setting. In particular, in Sect. 6.1 we analyze the structure of the terms contained in the definition $\mathcal{L}_{h}$ and its adjoint and provide a decomposition that isolates the crucial expressions. In Sect. 6.2 we show how our result can be established provided that a technical lower bound related to the sets $\left\{\left[\mathcal{L}_{h}-\delta\right] v\right\}_{\|v\|_{H^{1}}=1}$ can be obtained. We set out to derive this bound in Sect. 6.3, using a generalized version of the arguments in [1].

\subsection{Structure}

From now on, we simply write $\partial^{ \pm}, \partial^{0}$ and $\partial^{(2)}$ for the discrete derivatives if the value for $h$ is clear from the context. For any $w \in L^{2}$ and $h>0$, we introduce the function

$$
M_{h}^{\mathrm{adj}}[w]=c_{*} \partial^{0}\left[\gamma_{h}^{-1} w\right]-\partial^{0}\left[4 \gamma_{h}^{-4} \partial^{0} \Psi_{*}\left[\partial^{(2)} \Psi_{*}\right] w\right]+\partial^{(2)}\left[\gamma_{h}^{-2} w\right]+\gamma_{h}^{2} g^{\prime}\left(\Psi_{*}\right) w,
$$

together with the formal adjoint $\mathcal{L}_{h}^{\text {adj }}: H^{1} \rightarrow L^{2}$ that acts as

$$
\mathcal{L}_{h}^{\mathrm{adj}} w=c_{*} w^{\prime}-c_{*} \partial^{0} w+M_{h}^{\operatorname{adj}}[w]+M_{h}^{\operatorname{adj}}\left[\gamma_{h}^{-2}\left[\partial^{(2)} \Psi_{*}\right] \sum_{+; h} w \partial^{0} \Psi_{*}\right] .
$$

Indeed, one readily checks that for any pair $(v, w) \in L^{2} \times L^{2}$ we have

$$
\left\langle M_{h}[v], w\right\rangle_{L^{2}}=\left\langle v, M_{h}^{\operatorname{adj}}[w]\right\rangle_{L^{2}}
$$


In addition, the computation

$$
\begin{aligned}
& \left\langle\partial^{0} \Psi_{*} \sum_{-; h}\left[\gamma_{h}^{-2}\left[\partial^{(2)} \Psi_{*}\right] M_{h}[v]\right], w\right\rangle_{L^{2}}=\left\langle\gamma_{h}^{-2}\left[\partial^{(2)} \Psi_{*}\right] M_{h}[v], \sum_{+; h} w \partial^{0} \Psi_{*}\right\rangle_{L^{2}} \\
& =\left\langle M_{h}[v], \gamma_{h}^{-2}\left[\partial^{(2)} \Psi_{*}\right] \sum_{+; h} w \partial^{0} \Psi_{*}\right\rangle_{L^{2}} \\
& =\left\langle v, M_{h}^{\operatorname{adj}}\left[\gamma_{h}^{-2}\left[\partial^{(2)} \Psi_{*}\right] \sum_{+; h} w \partial^{0} \Psi_{*}\right]\right\rangle_{L^{2}}
\end{aligned}
$$

allows us to verify that

$$
\left\langle\mathcal{L}_{h} v, w\right\rangle_{L^{2}}=\left\langle v, \mathcal{L}_{h}^{\mathrm{adj}} w\right\rangle_{L^{2}}
$$

for any pair $(v, w) \in H^{1} \times H^{1}$.

Our goal here is to establish the following structural decomposition of $\mathcal{L}_{h}$ and $\mathcal{L}_{h}^{\text {adj }}$. Roughly speaking, this decomposition isolates all the terms that cannot be exponentially localized. In addition, it explicitly describes how the formal $h \downarrow 0$ limit can be related to twisted versions of the operators $\mathcal{L}_{\mathrm{cmp}}$ and $\mathcal{L}_{\mathrm{cmp}}^{\mathrm{adj}}$ that were discussed in $\S 3$.

Proposition 6.1 Suppose that $(\mathrm{Hg})$ and $\left(H \Phi_{*}\right)$ are satisfied and pick $\eta>0$ sufficiently small. There exists a constant $K>0$ together with linear maps

$$
L_{c ; h}: H^{1} \rightarrow L^{2}, \quad L_{c ; h}^{\text {adj }}: H^{1} \rightarrow L^{2},
$$

defined for all $0<h<1$, so that the following properties hold true.

(i) For every $0<h<1$ the identities

$$
\begin{aligned}
& \mathcal{L}_{h} v=-c_{*} v^{\prime}+\gamma_{h}^{-2} \partial^{(2)} v+\gamma_{h}^{2} g^{\prime}\left(\Psi_{*}\right) v+L_{c ; h}[v], \\
& \mathcal{L}_{h}^{\text {adj }} w=c_{*} w^{\prime}+\gamma_{h}^{-2} \partial^{(2)} w+\gamma_{h}^{2} g^{\prime}\left(\Psi_{*}\right) w+L_{c ; h}^{\text {adj }}[w]
\end{aligned}
$$

hold for all $v \in H^{1}$ and $w \in H^{1}$.

(ii) For any $0<h<1$ we have the bounds

$$
\| \begin{aligned}
& L_{c ; h}[v]\left\|_{L^{2}} \leq K\right\| v \|_{H^{1}}, \\
& L_{c ; h}^{\operatorname{adj}}[w]\left\|_{L^{2}} \leq K\right\| w \|_{H^{1}}
\end{aligned}
$$

for all $v \in H^{1}$ and $w \in H^{1}$.

(iii) For every $0<h<1$ we have the bounds

$$
\begin{aligned}
& \left\|e_{2 \eta}^{-1} L_{c ; h}[v]\right\|_{L_{\eta}^{2}} \leq K\left[\|v\|_{L_{\eta}^{2}}+\left\|\partial^{+} v\right\|_{L_{\eta}^{2}}\right], \\
& \left\|e_{2 \eta}^{-1} L_{c ; h}^{\operatorname{adj}}[w]\right\|_{L_{\eta}^{2}} \leq K\left[\|w\|_{L_{\eta}^{2}}+\left\|\partial^{+} w\right\|_{L_{\eta}^{2}}\right]
\end{aligned}
$$

for all $v \in H^{1}$ and $w \in H^{1}$.

(iv) Consider two sequences $\left\{\left(h_{j}, v_{j}\right)\right\}$ and $\left\{\left(h_{j}, w_{j}\right)\right\}$ that both satisfy the condition (hSeq) introduced in Sect. 4.3.

Then there exist two pairs $\left(V_{*}, W_{*}\right) \in H^{2} \times H^{2}$ and $\left(F_{*}, F_{*}^{\mathrm{adj}}\right) \in L^{2} \times L^{2}$ for which the weak convergences

$$
\left(v_{j}, \mathcal{L}_{h_{j}}\left[v_{j}\right]\right) \rightarrow\left(V_{*}, F_{*}\right) \in H^{1} \times L^{2}, \quad\left(w_{j}, \mathcal{L}_{h_{j}}^{\mathrm{adj}}\left[w_{j}\right]\right) \rightarrow\left(W_{*}, F_{*}^{\mathrm{adj}}\right) \in H^{1} \times L^{2}
$$


both hold, possibly after passing to a further subsequence. In addition, we have the identity

$$
\mathcal{L}_{\mathrm{cmp}} V_{*}=\mathcal{T}_{*} F_{*}
$$

and we have

$$
W_{*}=\mathcal{T}_{*}^{\mathrm{adj}} H_{*}
$$

for some $H_{*} \in H^{2}$ that satisfies

$$
\mathcal{L}_{\mathrm{cmp}}^{\mathrm{adj}}\left[H_{*}\right]=F_{*}^{\mathrm{adj}} .
$$

\section{Decomposition for $\mathcal{L}_{h}$}

We set out to identify all the terms in $\mathcal{L}_{h}$ that can be exponentially localized in the sense of (6.9). We start by analyzing the function $M_{h}[v]$, which can be treated by direct inspection.

Lemma 6.2 Suppose that $(H g)$ and $\left(H \Phi_{*}\right)$ are satisfied and pick $\eta>0$ sufficiently small. There exists a constant $K>0$ together with functions $\alpha_{0 ; h} \in H^{1}$, defined for $0<h<1$, so that the following properties hold.

(i) For every $0<h<1$ and $\tau \in \mathbb{R}$ we have

$$
\left|\alpha_{0 ; h}(\tau)\right| \leq K e_{2 \eta}(\tau) .
$$

(ii) For any $0<h<1$ and $v \in H^{1}$ we have the identity

$$
c_{*} \partial^{0} v+M_{h}[v]=\gamma_{h}^{-2} \partial^{(2)} v+\gamma_{h}^{2} g^{\prime}\left(\Psi_{*}\right) v+\alpha_{0 ; h} \partial^{0} v .
$$

(iii) For any sequence $\left\{\left(h_{j}, v_{j}\right)\right\}$ that satisfies (hSeq), there exists $V_{*} \in L^{2}$ for which the weak convergences

$$
v_{j} \rightarrow V_{*}, \quad M_{h_{j}}\left[v_{j}\right] \rightarrow \gamma_{*}^{2} \mathcal{L}_{\mathrm{cmp}}\left[V_{*}\right] \in L^{2}
$$

both hold as $j \rightarrow \infty$, possibly after passing to a subsequence.

Proof Writing

$$
\alpha_{0 ; h}=c_{*}\left(1-\gamma_{h}^{-1}\right)+4 \gamma_{h}^{-4}\left[\partial^{0} \Psi_{*}\right] \partial^{(2)} \Psi_{*},
$$

item (ii) follows by inspection. Item (i) follows from the exponential bounds (3.2) together with an application of the Lipschitz bound (A.7) with $U^{(a)}=0$ and $\gamma_{U^{(a)}}=1$.

Turning to (iii), we may exploit the fact that $\Psi_{*} \in H^{4}$ to apply the bounds in Sect. 4.1 and obtain the strong limits

$$
\gamma_{h}^{-2} \rightarrow \gamma_{*}^{-2} \in H^{1}, \quad \gamma_{h}^{2} g^{\prime}\left(\Psi_{*}\right) \rightarrow \gamma_{*}^{2} g^{\prime}\left(\Psi_{*}\right) \in H^{1},
$$

together with

$$
\alpha_{0 ; h_{j}} \rightarrow c_{*}\left(1-\gamma_{*}^{-1}\right)+2 \gamma_{*}^{-4} \Psi_{*}^{\prime} \Psi_{*}^{\prime \prime} \in H^{1} .
$$

In particular, we may apply Lemma's 4.6 and 4.7 to obtain the weak convergence

$$
M_{h_{j}}\left[v_{j}\right] \rightarrow-c_{*} \gamma_{*}^{-1} V_{*}^{\prime}+4 \gamma_{*}^{-4} \Psi_{*}^{\prime} \Psi_{*}^{\prime \prime} V_{*}^{\prime}+\gamma_{*}^{-2} V_{*}^{\prime \prime}+\gamma_{*}^{2} g^{\prime}\left(\Psi_{*}\right) V_{*} \in L^{2} .
$$

Inspecting the definition (2.12) yields (iii). 
It is convenient to introduce the notation

$$
\Upsilon_{h}[v]=\sum_{-; h} \gamma_{h}^{-2}\left[\partial^{(2)} \Psi_{*}\right] M_{h}[v],
$$

which in view of (6.16) allows us to obtain the expression (6.8) for $\mathcal{L}_{h}$ by writing

$$
L_{c ; h}[v]=\alpha_{0 ; h} \partial^{0} v+\left[\partial^{0} \Psi_{*}\right] \Upsilon_{h}[v] .
$$

Lemma 6.3 Suppose that $(H g)$ and $\left(H \Phi_{*}\right)$ are satisfied and pick $\eta>0$ sufficiently small. There exists a constant $K>0$ so that the following properties hold.

(i) For any $v \in H^{1}$ and $0<h<1$, we have the estimate

$$
\left\|\Upsilon_{h}[v]\right\|_{L_{\eta}^{2}} \leq K\left[\|v\|_{L_{\eta}^{2}}+\left\|\partial^{+} v\right\|_{L_{\eta}^{2}}\right]
$$

(ii) For any sequence $\left\{\left(h_{j}, v_{j}\right)\right\}$ that satisfies (hSeq), there exists $V_{*} \in L^{2}$ for which the weak convergences

$$
v_{j} \rightarrow V_{*}, \quad\left[\partial_{h_{j}}^{0} \Psi_{*}\right] \Upsilon_{h_{j}}\left[v_{j}\right] \rightarrow \Psi_{*}^{\prime} \int_{-} \Psi_{*}^{\prime \prime} \mathcal{L}_{\mathrm{cmp}} V_{*} \in L^{2}
$$

both hold as $j \rightarrow \infty$, possibly after passing to a subsequence.

Proof We make the splitting $\Upsilon_{h}[v]=\Upsilon_{A ; h}[v]+\Upsilon_{B ; h}[v]$ by introducing the notation

$$
\begin{aligned}
& \Upsilon_{A ; h}[v]=\sum_{-; h} \gamma_{h}^{-2} \partial^{(2)} \Psi_{*}\left[M_{h}[v]-\gamma_{h}^{-2} \partial^{(2)} v\right], \\
& \Upsilon_{B ; h}[v]=\sum_{-; h} \gamma_{h}^{-4}\left[\partial^{(2)} \Psi_{*}\right] \partial^{(2)} v .
\end{aligned}
$$

Applying Lemma 4.5 and inspecting (6.16), we see that

$$
\begin{aligned}
\left\|\Upsilon_{A ; h}[v]\right\|_{L_{\eta}^{2}} & \leq C_{1}^{\prime}\left\|M_{h}[v]-\gamma_{h}^{-2} \partial^{(2)} v\right\|_{L_{\eta}^{2}} \\
& \leq C_{2}^{\prime}\left[\|v\|_{L_{\eta}^{2}}+\left\|\partial^{+} v\right\|_{L_{\eta}^{2}}\right] .
\end{aligned}
$$

Applying the summation-by-parts identity (A.5), we compute

$$
\begin{aligned}
\Upsilon_{B ; h}[v] & =\sum_{-; h} \gamma_{h}^{-4}\left[\partial^{(2)} \Psi_{*}\right] \partial^{+} \partial^{-} v \\
& =T^{-}\left[\gamma_{h}^{-4} \partial^{(2)} \Psi_{*}\right] \partial^{-} v-\sum_{-; h}\left[\partial^{-} v\right] \partial^{-}\left[\gamma_{h}^{-4} \partial^{(2)} \Psi_{*}\right] .
\end{aligned}
$$

Item (i) now follows from a second application of Lemma 4.5.

To obtain (ii), we set out to apply Lemma 4.7 with $f_{j}=M_{h_{j}}\left[v_{j}\right], \alpha_{2 ; j}=\gamma_{h_{j}}^{-2} \partial_{h_{j}}^{(2)} \Psi_{*}$ and $\alpha_{1 ; j}=\partial_{h_{j}}^{0} \Psi_{*}$. Exploiting the fact that $\Psi_{*} \in H^{4}$, we may reason as in the proof of Lemma 6.2 to obtain the strong limits

$$
\alpha_{1 ; j} \rightarrow \Psi_{*}^{\prime} \in H^{1}, \quad \alpha_{2 ; j} \rightarrow \gamma_{*}^{-2} \Psi_{*}^{\prime \prime} \in H^{1} .
$$

Item (iii) of Lemma 6.2 implies that

$$
f_{*}=\gamma_{*}^{2} \mathcal{L}_{\mathrm{cmp}}\left[V_{*}\right],
$$

from which the desired weak limit follows. 


\section{Decomposition for $\mathcal{L}_{h}^{\text {adj }}$}

We set out to here to mimic the procedure above for $\mathcal{L}_{h}^{\text {adj }}$, which has a more convoluted structure. Special care needs to be taken to handle the fact that $M_{h}^{\text {adj }}$ acts on a discrete sum. The identities (A.4) play a crucial role here.

Lemma 6.4 Suppose that $(\mathrm{Hg})$ and $\left(H \Phi_{*}\right)$ are satisfied and pick $\eta>0$ sufficiently small. There exists a constant $K>0$ together with a set of functions

$$
\left(\alpha_{0 ; h}, \alpha_{0 s ; h}, \alpha_{+; h}, \alpha_{-; h}\right) \in H^{1} \times H^{1} \times H^{1} \times H^{1},
$$

defined for $0<h<1$, so that the following properties hold.

(i) For every $0<h<1$ and $\tau \in \mathbb{R}$ we have

$$
\left|\alpha_{0 ; h}(\tau)\right|+\left|\alpha_{0 s ; h}(\tau)\right|+\left|\alpha_{-; h}(\tau)\right|+\left|\alpha_{+; h}(\tau)\right| \leq K e_{2 \eta}(\tau) .
$$

(ii) For any $0<h<1$ and $w \in H^{1}$ we have the identity

$$
\begin{aligned}
-c_{*} \partial^{0} w+M_{h}^{\operatorname{adj}}[w]= & \gamma_{h}^{-2} \partial^{(2)} w+\gamma_{h}^{2} g^{\prime}\left(\Psi_{*}\right) w \\
& +\alpha_{0 ; h} w+\alpha_{0 s ; h} T^{+} w+\alpha_{+; h} \partial^{+} w+\alpha_{-; h} \partial^{-} w .
\end{aligned}
$$

(iii) For any sequence $\left\{\left(h_{j}, w_{j}\right)\right\}$ that satisfies (hSeq), there exists $W_{*} \in L^{2}$ for which the weak convergences

$$
w_{j} \rightarrow W_{*}, \quad M_{h_{j}}^{\operatorname{adj}}\left[w_{j}\right] \rightarrow \mathcal{L}_{\mathrm{cmp}}^{\mathrm{adj}}\left[\gamma_{*}^{2} W_{*}\right] \in L^{2}
$$

both hold as $j \rightarrow \infty$, possibly after passing to a subsequence.

Proof Applying (A.2) and (A.3), we obtain

$$
\begin{aligned}
M_{h}^{\mathrm{adj}}[w]= & c_{*} \partial^{0}\left[\gamma_{h}^{-1}\right] T^{+}[w]+c_{*} T^{-}\left[\gamma_{h}^{-1}\right] \partial^{0} w \\
& -4 \partial^{0}\left[\gamma_{h}^{-4}\left[\partial^{0} \Psi_{*}\right] \partial^{(2)} \Psi_{*}\right] T^{+} w-4 T^{-}\left[\gamma_{h}^{-4}\left[\partial^{0} \Psi_{*}\right] \partial^{(2)} \Psi_{*}\right] \partial^{0} w \\
& +\left[\partial^{(2)} \gamma_{h}^{-2}\right] w+\gamma_{h}^{-2} \partial^{(2)} w+\partial^{+}\left[\gamma_{h}^{-2}\right] \partial^{+} w+\partial^{-}\left[\gamma_{h}^{-2}\right] \partial^{-} w \\
& +\gamma_{h}^{2} g^{\prime}\left(\Psi_{*}\right) w
\end{aligned}
$$

from which (i) and (ii) can be read off.

Turning to (iii), we note first that the identity

$$
T^{+} w_{j}=w_{j}+h_{j} \partial^{+} w_{j}
$$

shows that also $T^{+} w_{j} \rightarrow W_{*} \in L^{2}$. Applying Lemma's 4.6 and 4.7 to the representation (6.35), we obtain the weak limit

$$
\begin{aligned}
M_{h_{j}}^{\mathrm{adj}}\left[w_{j}\right] \rightarrow & c_{*}\left[\gamma_{*}^{-1}\right]^{\prime} W_{*}+c_{*}\left[\gamma_{*}^{-1}\right] W_{*}^{\prime} \\
& -\left[4 \gamma_{*}^{-4} \Psi_{*}^{\prime} \Psi_{*}^{\prime \prime}\right]^{\prime} W_{*}-\left[4 \gamma_{*}^{-4} \Psi_{*}^{\prime} \Psi_{*}^{\prime \prime}\right] W_{*}^{\prime} \\
& +\left[\gamma_{*}^{-2}\right]^{\prime \prime} W_{*}+\gamma_{*}^{-2} W_{*}^{\prime \prime}+2\left[\gamma_{*}^{-2}\right]^{\prime} W_{*}^{\prime}+\gamma_{*}^{2} g^{\prime}\left(\Psi_{*}\right) W_{*} \\
= & c_{*} \partial_{\tau}\left[\gamma_{*}^{-1} W_{*}\right]-\partial_{\tau}\left[4 \gamma_{*}^{-4} \Psi_{*}^{\prime} \Psi_{*}^{\prime \prime} W_{*}\right]+\partial_{\tau \tau}\left[\gamma_{*}^{-2} W_{*}\right]+\gamma_{*}^{2} g^{\prime}\left(\Psi_{*}\right) W_{*} .
\end{aligned}
$$

Inspecting the definition (2.13) now yields the result. 
It is convenient to introduce the notation

$$
\Upsilon_{h}^{\mathrm{adj}}[w]=\sum_{+; h} w \partial^{0} \Psi_{*}
$$

which in view of (6.33) allows us to obtain the expression (6.8) for $\mathcal{L}_{h}^{\text {adj }}$ by writing

$$
\begin{aligned}
L_{c ; h}^{\operatorname{adj}}[w]= & \alpha_{0} w+\alpha_{0, s} T^{+} w+\alpha_{+} \partial^{+} w+\alpha_{-} \partial^{-} w \\
& +M_{h}^{\text {adj }}\left[\gamma_{h}^{-2}\left[\partial^{(2)} \Psi_{*}\right] \Upsilon_{h}^{\text {adj }}[w]\right] .
\end{aligned}
$$

Lemma 6.5 Suppose that $(H g)$ and $\left(H \Phi_{*}\right)$ are satisfied and pick $\eta>0$ sufficiently small. There exists a constant $K>0$ together with a set of functions

$$
\left(\tilde{\alpha}_{0 ; h}, \tilde{\alpha}_{0 s ; h}, \tilde{\alpha}_{+; h}, \tilde{\alpha}_{\omega ; h}, \tilde{\alpha}_{\omega s ; h}\right) \in H^{1} \times H^{1} \times H^{1} \times H^{1} \times H^{1},
$$

defined for $0<h<1$, so that the following properties hold.

(i) For any $0<h<1$ and $w \in H^{1}$, we have the estimate

$$
\left\|\Upsilon_{h}^{\operatorname{adj}}[w]\right\|_{L_{\eta}^{2}} \leq K\|w\|_{L_{\eta}^{2}} .
$$

(ii) For every $0<h<1$ and $\tau \in \mathbb{R}$ we have

$$
\left|\tilde{\alpha}_{0 ; h}(\tau)\right|+\left|\tilde{\alpha}_{0 s ; h}(\tau)\right|+\left|\tilde{\alpha}_{+; h}(\tau)\right|+\left|\tilde{\alpha}_{\omega ; h}(\tau)\right|+\left|\tilde{\alpha}_{\omega s ; h}(\tau)\right| \leq K e_{2 \eta}(\tau) .
$$

(iii) For every $0<h<1$ and $w \in H^{1}$, we have the identity

$$
\begin{aligned}
M_{h}^{\operatorname{adj}}\left[\gamma_{h}^{-2}\left[\partial^{(2)} \Psi_{*}\right] \Upsilon_{h}^{\operatorname{adj}}[w]\right]= & \tilde{\alpha}_{0 ; h} w+\tilde{\alpha}_{0 s ; h} T^{+} w+\tilde{\alpha}_{+; h} \partial^{+} w \\
& +\tilde{\alpha}_{\omega ; h} \Upsilon_{h}^{\text {adj }}[w]+\tilde{\alpha}_{\omega s ; h} T^{+} \Upsilon_{h}^{\text {adj }}[w] .
\end{aligned}
$$

(iv) For any sequence $\left\{\left(h_{j}, w_{j}\right)\right\}$ that satisfies (hSeq), there exists $W_{*} \in L^{2}$ for which the weak convergences

$$
w_{j} \rightarrow W_{*}, \quad M_{h_{j}}^{\operatorname{adj}}\left[\gamma_{h}^{-2}\left[\partial^{(2)} \Psi_{*}\right] \Upsilon_{h_{j}}^{\operatorname{adj}}\left[w_{j}\right]\right] \rightarrow \mathcal{L}_{\mathrm{cmp}}^{\operatorname{adj}}\left[\Psi_{*}^{\prime \prime} \int_{+} \Psi_{*}^{\prime} W_{*}\right] \in L^{2}(6.44)
$$

both hold as $j \rightarrow \infty$, possibly after passing to a subsequence.

Proof Item (i) can be obtained in a similar fashion as item (i) of Lemma 6.3. Using the identities (A.2)-(A.4), we compute

$$
\partial^{-}\left[\Upsilon_{h}^{\mathrm{adj}}[w]\right]=-w \partial^{0} \Psi_{*}
$$

and hence

$$
\begin{aligned}
\partial^{0}\left[\Upsilon_{h}^{\operatorname{adj}}[w]\right] & =-S^{+}\left[w \partial^{0} \Psi_{*}\right], \\
\partial^{(2)}\left[\Upsilon_{h}^{\text {adj }}[w]\right] & =\partial^{+} \partial^{-}\left[\Upsilon_{h}^{\text {adj }}[w]\right] \\
& =-\partial^{+}\left[\partial^{0} \Psi_{*}\right] T^{+} w-\left[\partial^{0} \Psi_{*}\right] \partial^{+} w .
\end{aligned}
$$

Writing

$$
\mathcal{I}[w]=\gamma_{h}^{-2}\left[\partial^{(2)} \Psi_{*}\right] \Upsilon_{h}^{\operatorname{adj}}[w],
$$

this gives

$$
\begin{aligned}
\partial^{0}[\mathcal{I}[w]]= & \partial^{0}\left[\gamma_{h}^{-2} \partial^{(2)} \Psi_{*}\right] T^{+}\left[\Upsilon_{h}^{\mathrm{adj}}[w]\right] \\
& -T^{-}\left[\gamma_{h}^{-2}\left[\partial^{(2)} \Psi_{*}\right]\right] S^{+}\left[w \partial^{0} \Psi_{*}\right]
\end{aligned}
$$


together with

$$
\begin{aligned}
\partial^{(2)}[\mathcal{I}[w]]= & \partial^{(2)}\left[\gamma_{h}^{-2} \partial^{(2)} \Psi_{*}\right]\left[\Upsilon_{h}^{\text {adj }}[w]\right] \\
& +\left[\gamma_{h}^{-2} \partial^{(2)} \Psi_{*}\right]\left[-\partial^{+}\left[\partial^{0} \Psi_{*}\right] T^{+} w-\left[\partial^{0} \Psi_{*}\right] \partial^{+} w\right] \\
& +\partial^{+}\left[\gamma_{h}^{-2} \partial^{(2)} \Psi_{*}\right] T^{+}\left[-w \partial^{0} \Psi_{*}\right] \\
& +\partial^{-}\left[\gamma_{h}^{-2} \partial^{(2)} \Psi_{*}\right]\left[-w \partial^{0} \Psi_{*}\right] .
\end{aligned}
$$

Items (ii) and (iii) can now be read off from the representation (6.33) and the exponential bounds (3.2).

Suppose now that $\left\{\left(h_{j}, w_{j}\right)\right\}$ satisfies (hSeq) and write

$$
\mathcal{I}_{j}=\gamma_{h_{j}}^{-2}\left[\partial_{h_{j}}^{(2)} \Psi_{*}\right] \Upsilon_{h_{j}}^{\mathrm{adj}}\left[w_{j}\right] .
$$

Using the same arguments as in the proof of item (ii) of Lemma 6.3, we can apply Lemma 4.7 to obtain the weak convergence

$$
\mathcal{I}_{j} \rightarrow \gamma_{*}^{-2} \Psi_{*}^{\prime \prime} \int_{+} \Psi_{*} W_{*} \in L^{2} .
$$

In addition, using the identity

$$
\left[\Upsilon_{h}^{\operatorname{adj}}[w]\right]^{\prime}=\sum_{+; h}\left[w \partial^{0} \Psi_{*}^{\prime}+w^{\prime} \partial^{0} \Psi_{*}\right]
$$

together with Lemma 4.5 , we see that $\left\|\mathcal{I}_{j}\right\|_{H^{1}}$ can be uniformly bounded. Finally, (6.49) together with the fact that $\Psi_{*} \in H^{5}$ implies that also $\left\|\partial^{+} \partial^{+} \mathcal{I}_{j}\right\|_{L^{2}}$ can be uniformly bounded. In particular, the sequence $\left\{\left(h_{j}, \mathcal{I}_{j}\right)\right\}$ also satisfies (hSeq). Applying item (iii) of Lemma 6.4 now yields (iv).

Proof of Proposition 6.1 Items (i) and (ii) follow directly from Lemma's 6.2, 6.3, 6.4 and 6.5. Under the assumptions of (iii), the weak limits (6.11) follow from the fact that $\left\{\mathcal{L}_{h_{j}}\left[v_{j}\right]\right\}$ and $\left\{\mathcal{L}_{h_{j}}^{\mathrm{adj}}\left[w_{j}\right]\right\}$ are bounded sequences in $L^{2}$. Using Lemma's 6.2 and 6.3, we see that

$$
F_{*}=\gamma_{*}^{2} \mathcal{L}_{\mathrm{cmp}}\left[V_{*}\right]+\Psi_{*}^{\prime} \int_{-} \Psi_{*}^{\prime \prime} \mathcal{L}_{\mathrm{cmp}}\left[V_{*}\right]
$$

Applying (3.72) yields (6.12).

On the other hand, Lemma's 6.4 and 6.5 show that

$$
F_{*}^{\mathrm{adj}}=\mathcal{L}_{\mathrm{cmp}}^{\mathrm{adj}}\left[\gamma_{*}^{2} W_{*}\right]+\mathcal{L}_{\mathrm{cmp}}^{\mathrm{adj}}\left[\Psi_{*}^{\prime \prime} \int_{+} \Psi_{*}^{\prime} W_{*}\right]
$$

In particular, we can satisfy (6.14) by writing

$$
H_{*}=\gamma_{*}^{2} W_{*}+\Psi_{*}^{\prime \prime} \int_{+} \Psi_{*}^{\prime} W_{*} .
$$

Applying (3.73) we see that

$$
W_{*}=\mathcal{T}_{*}^{\text {adj }} H_{*},
$$

as desired. 


\subsection{Strategy}

In this subsection we show that Theorem 2.3 can be established by finding appropriate lower bounds for the quantities

$$
\begin{aligned}
& \mathcal{E}_{h}(\delta)=\inf _{\|v\|_{H^{1}}=1}\left\{\left\|\mathcal{L}_{h} v-\delta v\right\|_{L^{2}}+\delta^{-1}\left|\left\langle\Psi_{*}^{\mathrm{adj}}, \mathcal{T}_{*}\left[\mathcal{L}_{h} v-\delta v\right]\right\rangle_{L^{2}}\right|\right\}, \\
& \mathcal{E}_{h}^{\text {adj }}(\delta)=\inf _{\|w\|_{H^{1}}=1}\left\{\left\|\mathcal{L}_{h}^{\mathrm{adj}} w-\delta w\right\|_{L^{2}}+\delta^{-1}\left|\left\langle\Psi_{*}^{\prime}, \mathcal{L}_{h}^{\mathrm{adj}} w-\delta w\right\rangle_{L^{2}}\right|\right\} .
\end{aligned}
$$

In particular, the required bounds are formulated in the following result, which is analogous to $[1$, Lem. 6].

Proposition 6.6 Suppose that $(\mathrm{Hg})$ and $\left(H \Phi_{*}\right)$ are satisfied. Then there exists $\mu>0$ and $\delta_{0}>0$ such that for every $0<\delta<\delta_{0}$ we have

$$
\begin{array}{ll}
\mu(\delta) & :=\liminf _{h \downarrow 0} \mathcal{E}_{h}(\delta) \geq \mu, \\
\mu^{\operatorname{adj}}(\delta) & :=\liminf _{h \downarrow 0} \mathcal{E}_{h}^{\operatorname{adj}}(\delta) \geq \mu .
\end{array}
$$

We postpone the proof of this result to Sect. 6.3, but set out to explore the consequences here. In particular, it enables us to show that the operators $\mathcal{L}_{h}-\delta$ are invertible for small $h>0$ and $\delta>0$, providing us with the analogue of [1, Thm. 4].

Proposition 6.7 Suppose that $(\mathrm{Hg})$ and $\left(H \Phi_{*}\right)$ are satisfied. There exists constants $K>0$ and $\delta_{0}>0$ together with a map $h_{0}:\left(0, \delta_{0}\right) \rightarrow(0,1)$ so that the following holds true. For any $0<\delta<\delta_{0}$ and any $0<h<h_{0}(\delta)$, the operator $\mathcal{L}_{h}-\delta$ is invertible as a map from $H^{1}$ onto $L^{2}$ and satisfies the bound

$$
\left\|\left(\mathcal{L}_{h}-\delta\right)^{-1} f\right\|_{H^{1}} \leq K\left[\|f\|_{L^{2}}+\delta^{-1}\left|\left\langle\Psi_{*}^{\mathrm{adj}}, \mathcal{T}_{*} f\right\rangle\right|\right] .
$$

Proof Following the proof of [1, Thm. 4], we fix $0<\delta<\delta_{0}$ and a sufficiently small $h>0$. By Proposition 6.6, the operator $\mathcal{L}_{h}-\delta$ is an homeomorphism from $H^{1}$ onto its range

$$
\mathcal{R}=\left(\mathcal{L}_{h}-\delta\right)\left(H^{1}\right) \subset L^{2},
$$

with a bounded inverse $\mathcal{I}: \mathcal{R} \rightarrow H^{1}$. The latter fact shows that $\mathcal{R}$ is a closed subset of $L^{2}$. If $\mathcal{R} \neq L^{2}$, there exists a non-zero $w \in L^{2}$ so that $\langle w, \mathcal{R}\rangle_{L^{2}}=0$, i.e.,

$$
\left\langle w,\left(\mathcal{L}_{h}-\delta\right) v\right\rangle_{L^{2}}=0 \text { for all } v \in H^{1} .
$$

Restricting this identity to test functions $v \in C_{c}^{\infty}$ implies that in fact $w \in H^{1}$. In particular, we find

$$
\left\langle\left(\mathcal{L}_{h}^{\text {adj }}-\delta\right) w, v\right\rangle_{L^{2}}=0 \text { for all } v \in H^{1},
$$

which by the density of $H^{1}$ in $L^{2}$ means that $\left(\mathcal{L}_{h}^{\text {adj }}-\delta\right) w=0$. Applying Proposition 6.6 once more yields the contradiction $w=0$ and establishes $\mathcal{R}=L^{2}$. The bound (6.59) with the $\delta$-independent constant $K>0$ now follows directly from the definition (6.57) of the quantities $\mathcal{E}_{h}(\delta)$ and the uniform lower bound (6.58).

Following the ideas in [6, Sect. 3.3], we can take the $\delta \downarrow 0$ limit and establish our main result concerning $\mathcal{L}_{h}$. The bounds in (ii) rely heavily on the preliminary work in Sect. 5 related to the quantity

$$
\partial^{+} \mathcal{L}_{h}[v]-\mathcal{L}_{h}\left[\partial^{+} v\right]
$$


Proof of Theorem 2.3 For convenience, we introduce the set

$$
\mathcal{Z}_{h}=\left\{v \in H^{1}:\left\langle\Psi_{*}^{\text {adj }}, \mathcal{T}_{*} v\right\rangle_{L^{2}}=0\right\} .
$$

Our goal is to find, for any $f \in L^{2}$, a solution $(\beta, v) \in \mathbb{R} \times \mathcal{Z}_{h}$ to the problem

$$
v=\mathcal{V}_{h ; \delta}[f, v, \beta]:=\left(\mathcal{L}_{h}-\delta\right)^{-1}\left[f+\beta \Psi_{*}^{\prime}-\delta v\right] .
$$

In order to ensure that the linear operator $\mathcal{V}_{h ; \delta}$ indeed maps into $\mathcal{Z}_{h}$, it suffices to choose $\beta$ in such a way that

$$
\beta\left\langle\Psi_{*}^{\mathrm{adj}}, \mathcal{T}_{*}\left(\mathcal{L}_{h}-\delta\right)^{-1} \Psi_{*}^{\prime}\right\rangle_{L^{2}}=-\left\langle\Psi_{*}^{\mathrm{adj}}, \mathcal{T}_{*}\left(\mathcal{L}_{h}-\delta\right)^{-1}(f-\delta v)\right\rangle_{L^{2}} .
$$

Writing

$$
\left(\mathcal{L}_{h}-\delta\right)^{-1} \Psi_{*}^{\prime}=-\delta^{-1} \Psi_{*}^{\prime}+\bar{v}
$$

we see that

$$
\left[\mathcal{L}_{h}-\delta\right] \bar{v}=\delta^{-1} \mathcal{L}_{h} \Psi_{*}^{\prime},
$$

which shows that

$$
\|\bar{v}\|_{H^{1}} \leq C_{1}^{\prime} h \delta^{-2} .
$$

Choosing $\delta<1$ and recalling the normalization

$$
\left\langle\Psi^{\mathrm{adj}}, \mathcal{T}_{*} \Psi_{*}^{\prime}\right\rangle_{L^{2}}=1,
$$

we can impose a restriction $h \leq\left[C_{2}^{\prime}\right]^{-1} \delta^{2}$ to ensure that

$$
\left|\left\langle\Psi_{*}^{\text {adj }}, \mathcal{T}_{*}\left(\mathcal{L}_{h}-\delta\right)^{-1} \Psi_{*}^{\prime}\right\rangle_{L^{2}}\right| \geq \frac{1}{2} \delta^{-1} .
$$

In particular, we can find a unique solution $\beta=\beta_{h ; \delta}[f, v]$ to (6.66) for every $v \in \mathcal{Z}_{h}$ and $f \in L^{2}$.

The definition of $\mathcal{Z}_{h}$ implies the bound

$$
\left\|\left(\mathcal{L}_{h}-\delta\right)^{-1}(f-\delta v)\right\| \leq C_{3}^{\prime}\left[\delta^{-1}\|f\|_{L^{2}}+\delta\|v\|_{L^{2}}\right],
$$

which allows us to obtain the estimate

$$
\left|\beta_{h ; \delta}[f, v]\right| \leq C_{4}^{\prime}\left[\|f\|_{L^{2}}+\delta^{2}\|v\|_{L^{2}}\right] .
$$

This in turn leads to the estimate

$$
\left\|\mathcal{V}_{h ; \delta}\left[f, v, \beta_{h ; \delta}[f, v]\right]\right\|_{H^{1}} \leq C_{5}^{\prime}\left[\delta^{-1}\|f\|_{L^{2}}+\delta\|v\|_{L^{2}}\right] .
$$

By choosing $\delta>0$ to be sufficiently small, we hence see that the linear fixed point problem

$$
v=\mathcal{V}_{h ; \delta}\left[f, v, \beta_{h ; \delta}[f, v]\right]
$$

posed on $\mathcal{Z}_{h}$ has a unique solution for all $f \in L^{2}$. Writing $v=\mathcal{V}_{h ; \delta}^{*} f$ for this solution together with

$$
\beta_{h ; \delta}^{*} f=\beta_{h ; \delta}\left[f, \mathcal{V}_{h ; \delta}^{*} f\right]
$$

we obtain the estimates

$$
\left\|\mathcal{V}_{h ; \delta}^{*} f\right\|_{H^{1}} \leq C_{6}^{\prime} \delta^{-1}\|f\|_{L^{2}}, \quad\left|\beta_{h ; \delta}^{*} f\right| \leq C_{6}^{\prime}\|f\|_{L^{2}} .
$$


The remarks above show that the problem (2.33)-(2.34) is equivalent to (6.75). We can hence fix a sufficiently small $\delta>0$ and write $\beta_{h}^{*}=\beta_{h ; \delta}^{*}$ and $\mathcal{V}_{h}^{*}=\mathcal{V}_{h ; \delta}^{*}$, which are well-defined for all sufficiently small $h>0$. This establishes (iii). Item (iv) can be verified directly by noting that $(v, \beta)=(0,-1)$ is a solution to (2.33)-(2.34) for $f=\Psi_{*}^{\prime}$.

Turning to (i) and (ii), let us pick $f \in L^{2}$ and write

$$
(v, \beta)=\left(\mathcal{V}_{h}^{*}[f], \beta_{h}^{*}[f]\right) .
$$

Item (iii) together with the representation (6.8) implies that

$$
c v^{\prime}+f+\beta \Psi_{*}^{\prime}=\gamma_{h}^{-2} \partial^{(2)} v+\gamma_{h}^{2} g^{\prime}\left(\Psi_{*}\right) v+L_{c ; h}[v] .
$$

The bound (i) follows from (6.77) and item (ii) of Proposition 6.1, which together provide $L^{2}$-bounds on all the terms in (6.79) that do not involve $\partial^{(2)} v$. To see (ii), we compute

$$
\mathcal{L}_{h}\left[\partial^{+} v\right]=\partial^{+}[f]+\beta \partial^{+}\left[\Psi_{*}^{\prime}\right]+\mathcal{L}_{h}\left[\partial^{+} v\right]-\partial^{+}\left[\mathcal{L}_{h}[v]\right]
$$

and note that Corollary 5.4 implies that

$$
\begin{aligned}
\left\|\mathcal{L}_{h}\left[\partial^{+} v\right]-\partial^{+}\left[\mathcal{L}_{h}[v]\right]\right\|_{L^{2}} & \leq C_{7}^{\prime}\left[\|v\|_{H^{1}}+\left\|\partial^{+} \partial^{+} v\right\|_{L^{2}}\right] \\
& \leq C_{8}^{\prime}\|f\|_{L^{2}} .
\end{aligned}
$$

Using (i) we conclude that

$$
\left\|\partial^{+} v\right\|_{H^{1}}+\left\|\partial^{+} \partial^{+} \partial^{+} v\right\|_{L^{2}} \leq C_{9}^{\prime}\left[\|f\|_{L^{2}}+\left\|\partial^{+} f\right\|_{L^{2}}\right]
$$

which establishes (ii).

\subsection{Proof of Proposition 6.6}

We set out here to obtain lower bounds for the quantities (6.57). As a first step, we show that the limiting values can be approached via a sequence of realizations for which the weak limits described in (iv) of Proposition 6.1 hold and for which the full power of Lemma 4.6 is available.

Lemma 6.8 Consider the setting of Proposition 6.6 and fix $0<\delta<\delta_{0}$. Then there exist four functions

$$
\left(V_{*}, W_{*}\right) \in H^{2} \times H^{2}, \quad\left(Y_{*}, Z_{*}\right) \in L^{2}(\mathbb{R}),
$$

together with a sequence

$$
\left\{\left(h_{j}, v_{j}, y_{j}, w_{j}, z_{j}\right)\right\}_{j \in \mathbb{N}} \subset(0,1) \times H^{1} \times L^{2} \times H^{1} \times L^{2}
$$

that satisfies the following properties.

(i) For any $j \in \mathbb{N}$, we have

$$
\left\|v_{j}\right\|_{H^{1}}=\left\|w_{j}\right\|_{H^{1}}=1
$$

together with

$$
\begin{aligned}
& \mathcal{L}_{h_{j}}\left[v_{j}\right]-\delta v_{j}=y_{j}, \\
& \mathcal{L}_{h_{j}}^{\text {adj }}\left[w_{j}\right]-\delta w_{j}=z_{j} .
\end{aligned}
$$


(ii) Recalling the constants $\left(\mu(\delta), \mu^{\operatorname{adj}}(\delta)\right)$ defined in (6.58), we have the limits

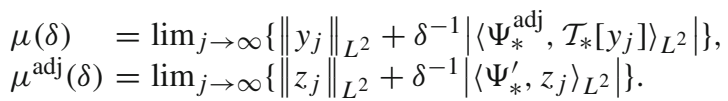

(iii) As $j \rightarrow \infty$, we have the weak convergences

$$
v_{j} \rightarrow V_{*} \in H^{1}, \quad w_{j} \rightarrow W_{*} \in H^{1},
$$

together with

$$
y_{j} \rightarrow Y_{*} \in L^{2}, \quad z_{j} \rightarrow Z_{*} \in L^{2} .
$$

(iv) The pairs $\left\{\left(h_{j}, v_{j}\right)\right\}$ and $\left\{\left(h_{j}, w_{j}\right)\right\}$ both satisfy $(h$ Seq $)$.

Proof The existence of the sequences (6.84) that satisfy (i) and (ii) with $h_{j} \downarrow 0$ follows directly from the definitions (6.58). Notice that (6.87) implies that we can pick $C_{1}>0$ for which we have the uniform bound

$$
\left\|y_{j}\right\|_{L^{2}}+\left\|z_{j}\right\|_{L^{2}} \leq C_{1}
$$

for all $j \in \mathbb{N}$. In particular, after taking a subspace we obtain (iii). In addition, item (ii) of Proposition 6.1 implies that also

$$
\left\|\partial_{h_{j}}^{(2)} v_{j}\right\|_{L^{2}}+\left\|\partial_{h_{j}}^{(2)} w_{j}\right\|_{L^{2}} \leq C_{2}
$$

for some $C_{2}>0$ and all $j>0$, which implies (iv).

Lemma 6.9 Consider the setting of Proposition 6.6. There exists a constant $K_{1}>0$ so that for any $0<\delta<\delta_{0}$, the function $V_{*}$ defined in Lemma 6.8 satisfies the bound

$$
\left\|V_{*}\right\|_{H^{2}} \leq K_{1} \mu(\delta) .
$$

Proof Item (iv) of Proposition 6.1 implies that

$$
\mathcal{L}_{\mathrm{cmp}}\left[V_{*}\right]=\mathcal{T}_{*}\left[Y_{*}+\delta V_{*}\right],
$$

which we rewrite as

$$
\mathcal{L}_{\mathrm{cmp}}\left[V_{*}\right]-\delta \mathcal{T}_{*}\left[V_{*}\right]=\mathcal{T}_{*} Y_{*} .
$$

The lower-semicontinuity of the $L^{2}$-norm under weak limits implies that

$$
\left\|Y_{*}\right\|_{L^{2}}+\delta^{-1}\left|\left\langle\Psi_{*}^{\mathrm{adj}}, \mathcal{T}_{*} Y_{*}\right\rangle\right| \leq \mu(\delta),
$$

while Lemma 3.12 implies that

$$
\left\|\mathcal{T}_{*} Y_{*}\right\|_{L^{2}}+\delta^{-1}\left|\left\langle\Psi_{*}^{\mathrm{adj}}, \mathcal{T}_{*} Y_{*}\right\rangle\right| \leq C_{1}^{\prime} \mu(\delta) .
$$

The desired bound hence follows directly from Corollary 3.2.

Lemma 6.10 Consider the setting of Proposition 6.6. There exists a constant $K_{1}>0$ so that for any $0<\delta<\delta_{0}$, the function $W_{*}$ defined in Lemma 6.8 satisfies the bound

$$
\left\|W_{*}\right\|_{H^{2}} \leq K_{1} \mu^{\operatorname{adj}}(\delta) .
$$


Proof Item (iv) of Proposition 6.1 implies that

$$
W_{*}=\mathcal{T}_{*}^{\mathrm{adj}} H_{*}
$$

for some $H_{*} \in H^{2}$ that satisfies the identity

$$
\mathcal{L}_{\mathrm{cmp}}^{\mathrm{adj}}\left[H_{*}\right]=\left[Z_{*}+\delta W_{*}\right]=\left[Z_{*}+\delta \mathcal{T}_{*}^{\mathrm{adj}} H_{*}\right] .
$$

In particular, we find

$$
\mathcal{L}_{\mathrm{cmp}}^{\mathrm{adj}}\left[H_{*}\right]-\delta \mathcal{T}_{*}^{\operatorname{adj}}\left[H_{*}\right]=Z_{*} .
$$

The lower-semicontinuity of the $L^{2}$-norm under weak limits implies that

$$
\left\|Z_{*}\right\|_{L^{2}}+\delta^{-1}\left|\left\langle\Psi_{*}^{\prime}, Z_{*}\right\rangle\right| \leq \mu(\delta) .
$$

Proposition 3.2 hence yields

$$
\left\|H_{*}\right\|_{H^{2}} \leq C_{1}^{\prime} \mu(\delta) \text {. }
$$

The desired bound hence follows from (6.98) and Lemma 3.12.

The next result controls the size of the derivatives $\left(v_{j}^{\prime}, w_{j}^{\prime}\right)$, which is crucial to rule out the leaking of energy into oscillations that are not captured by the relevant weak limits. The key novel element here compared to the setting in [1] is that one needs to include $\partial^{+} v_{j}$ in the bound. Our preparatory work enables us to measure this contribution in a weighted norm, which allows us to capture the bulk of the contribution on a compact interval.

Lemma 6.11 Consider the setting of Lemma 6.8 and pick a sufficiently small $\eta>0$. There exists a constant $K_{2}>1$ that does not depend on $0<\delta<\delta_{0}$ so that the inequalities

$$
\begin{aligned}
& \left\|v_{j}^{\prime}\right\|_{L_{2}}^{2} \leq K_{2}\left[\left\|y_{j}\right\|_{L^{2}}^{2}+\left\|v_{j}\right\|_{L^{2}}^{2}+\left\|\partial^{+} v_{j}\right\|_{L_{\eta}^{2}}^{2}\right], \\
& \left\|w_{j}^{\prime}\right\|_{L_{2}}^{2^{2}} \leq K_{2}\left[\left\|z_{j}\right\|_{L^{2}}^{2}+\left\|w_{j}\right\|_{L^{2}}^{2}+\left\|\partial^{+} w_{j}\right\|_{L_{\eta}^{2}}^{2}\right]
\end{aligned}
$$

hold for all $j>0$.

Proof Using the representation in item (i) of Proposition 6.1, we expand the identity

$$
\left\langle\mathcal{L}_{h_{j}} v_{j}-\delta v_{j}, \gamma_{h_{j}}^{2} v_{j}^{\prime}\right\rangle_{L^{2}}=\left\langle y_{j}, \gamma_{h_{j}}^{2} v_{j}^{\prime}\right\rangle_{L^{2}}
$$

to obtain

$$
\begin{aligned}
c_{*}\left\langle\gamma_{h_{j}}^{2} v_{j}^{\prime}, v_{j}^{\prime}\right\rangle_{L^{2}}+\left\langle y_{j}, \gamma_{h_{j}}^{2} v_{j}^{\prime}\right\rangle_{L^{2}}= & -\delta\left\langle v_{j}, \gamma_{h_{j}}^{2} v_{j}^{\prime}\right\rangle_{L^{2}}+\left\langle\partial^{(2)} v_{j}, v_{j}^{\prime}\right\rangle_{L^{2}}+\left\langle\gamma_{h_{j}}^{4} g^{\prime}\left(\Psi_{*}\right) v_{j}, v_{j}^{\prime}\right\rangle_{L^{2}} \\
& +\left\langle L_{c ; h_{j}}\left[v_{j}\right], \gamma_{h_{j}}^{2} v_{j}^{\prime}\right\rangle_{L^{2}} .
\end{aligned}
$$

Applying (4.24) together with item (iii) of Proposition 6.1, we note that

$$
\begin{aligned}
\left|\left\langle L_{c ; h_{j}}\left[v_{j}\right], \gamma_{h}^{2} v_{j}^{\prime}\right\rangle_{L^{2}}\right| & =\left|\left\langle e_{2 \eta}^{-1} L_{c ; h_{j}}\left[v_{j}\right], e_{2 \eta} \gamma_{h}^{2} v_{j}^{\prime}\right\rangle_{L^{2}}\right| \\
& \leq\left\|e_{2 \eta}^{-1} L_{c ; h_{j}}\left[v_{j}\right]\right\|_{L_{\eta}^{2}}\left\|\gamma_{h}^{2} v_{j}^{\prime}\right\|_{L_{\eta}^{2}} \\
& \leq C_{1}^{\prime}\left[\left\|v_{j}\right\|_{L_{\eta}^{2}}+\left\|\partial^{+} v_{j}\right\|_{L_{\eta}^{2}}\right]\left\|v_{j}^{\prime}\right\|_{L_{\eta}^{2}} .
\end{aligned}
$$


Using the identity $\left\langle\partial^{(2)} v_{j}, v_{j}^{\prime}\right\rangle_{L^{2}}=0$ together with the lower bound $\gamma_{h_{j}}^{2} \geq\left[C_{2}^{\prime}\right]^{-1}$ we may hence compute

$$
\begin{aligned}
\left|c_{*}\right|\left\langle v_{j}^{\prime}, v_{j}^{\prime}\right\rangle_{L^{2}} \leq & \left|c_{*}\right| C_{2}^{\prime}\left\langle\gamma_{h_{j}}^{2} v_{j}^{\prime}, v_{j}^{\prime}\right\rangle_{L^{2}} \\
\leq & C_{3}^{\prime}\left[\left\|v_{j}\right\|_{L^{2}}\left\|v_{j}^{\prime}\right\|_{L^{2}}+\left\|y_{j}\right\|_{L^{2}}\left\|v_{j}^{\prime}\right\|_{L^{2}}+\left\|v_{j}\right\|_{L_{\eta}^{2}}\left\|v_{j}^{\prime}\right\|_{L_{\eta}^{2}}\right. \\
& \left.+\left\|\partial^{+} v_{j}\right\|_{L_{\eta}^{2}}\left\|v_{j}^{\prime}\right\|_{L_{\eta}^{2}}\right] .
\end{aligned}
$$

Recalling the bound $\|a\|_{L_{\eta}^{2}} \leq\|a\|_{L^{2}}$ for $a \in L^{2}$ and using $c_{*} \neq 0$, we find

$$
\left\|v_{j}^{\prime}\right\|_{L^{2}}^{2} \leq C_{4}^{\prime}\left[\left\|v_{j}\right\|_{L^{2}}+\left\|y_{j}\right\|_{L^{2}}+\left\|\partial^{+} v_{j}\right\|_{L_{\eta}^{2}}\right]\left\|v_{j}^{\prime}\right\|_{L^{2}} .
$$

Dividing through by $\left\|v_{j}^{\prime}\right\|_{L^{2}}$ and squaring, we obtain

$$
\left\|v_{j}^{\prime}\right\|_{L_{2}}^{2} \leq C_{5}^{\prime}\left[\left\|v_{j}\right\|_{L^{2}}^{2}+\left\|y_{j}\right\|_{L^{2}}^{2}+\left\|\partial^{+} v_{j}\right\|_{L_{\eta}^{2}}^{2}\right]
$$

The same procedure works for $w_{j}^{\prime}$.

We are now almost ready to obtain lower bounds for $\left\|V_{*}\right\|_{H^{1}}$ and $\left\|W_{*}\right\|_{H^{1}}$, exploiting the fact that our nonlinearity is bistable. The next technical result is the analogue of the inequality $\left\langle\partial^{(2)} u, u\right\rangle_{L^{2}} \leq 0$ used in [1]. Due to the non-autonomous coefficient in front of the second difference, we obtain localized correction terms that need to be controlled.

Lemma 6.12 Suppose that $(H g)$ and $\left(H \Phi_{*}\right)$ are satisfied. There exists a constant $K>0$ so that for any $v \in H^{1}$ and any $0<h<1$, we have the one-sided inequality

$$
\left\langle\gamma_{h}^{-2} \partial^{(2)} v, v\right\rangle_{L^{2}} \leq K\left[\left\|\partial^{+} v\right\|_{L_{\eta}^{2}}^{2}+\|v\|_{L_{\eta}^{2}}^{2}\right] .
$$

Proof Using (A.2) we compute

$$
\begin{aligned}
-\left\langle\gamma_{h}^{-2} \partial^{(2)} v, v\right\rangle_{L^{2}}= & -\left\langle\gamma_{h}^{-2} \partial^{-} \partial^{+} v, v\right\rangle_{L^{2}} \\
= & \left\langle\partial^{+} v, \partial^{+}\left[\gamma_{h}^{-2} v\right]\right\rangle_{L^{2}} \\
= & \left\langle\partial^{+} v, \partial^{+}\left[\gamma_{h}^{-2}\right] T^{+}[v]\right\rangle_{L^{2}}+\left\langle\partial^{+} v, \gamma_{h}^{-2} \partial^{+} v\right\rangle_{L^{2}} \\
= & \left\langle\partial^{+} v, \partial^{+}\left[\gamma_{h}^{-2}\right] T^{+}[v]\right\rangle_{L^{2}}+\left\langle\partial^{+} v,\left(\gamma_{h}^{-2}-1\right) \partial^{+} v\right\rangle_{L^{2}} \\
& +\left\langle\partial^{+} v, \partial^{+} v\right\rangle_{L^{2}} \\
\geq & \left\langle\partial^{+} v, \partial^{+}\left[\gamma_{h}^{-2}\right] T^{+}[v]\right\rangle_{L^{2}}+\left\langle\partial^{+} v,\left(\gamma_{h}^{-2}-1\right) \partial^{+} v\right\rangle_{L^{2}} .
\end{aligned}
$$

The result now follows from (4.24) together with the pointwise exponential bounds

$$
\left|\gamma_{h}^{-2}-1\right|+\left|\partial^{+}\left[\gamma_{h}^{-2}\right]\right| \leq C_{1}^{\prime} e_{2 \eta} .
$$

Lemma 6.13 Consider the setting of Proposition 6.6. There exists constants $K_{2}>0$ and $K_{3}>0$ so that for any $0<\delta<\delta_{0}$, the functions $V_{*}$ and $W_{*}$ defined in Lemma 6.8 satisfy the bounds

$$
\begin{aligned}
& \left\|V_{*}\right\|_{H^{1}}^{2} \geq K_{3}-K_{4} \mu(\delta)^{2}, \\
& \left\|W_{*}\right\|_{H^{1}}^{2} \geq K_{3}-K_{4} \mu^{\operatorname{adj}}(\delta)^{2} .
\end{aligned}
$$


Proof Pick $m>1$ and $\alpha>0$ in such a way that

$$
\gamma_{h_{j}}^{2}(\tau) g^{\prime}\left(\Psi_{*}(\tau)\right) \leq-\alpha
$$

holds for all $|\tau| \geq m$. This is possible on account of the uniform lower bound $\gamma_{h}^{2} \geq\left[C_{1}^{\prime}\right]^{-1}$ and the fact that $g^{\prime}(0)<0$ and $g^{\prime}(1)<0$.

We now expand the identity

$$
\left\langle\mathcal{L}_{h_{j}} v_{j}-\delta v_{j}, v_{j}\right\rangle_{L^{2}}=\left\langle y_{j}, v_{j}\right\rangle_{L^{2}}
$$

to obtain the estimate

$$
\begin{aligned}
\left\langle y_{j}, v_{j}\right\rangle_{L^{2}}= & -c_{*}\left\langle v_{j}^{\prime}, v_{j}\right\rangle_{L^{2}}-\delta\left\langle v_{j}, v_{j}\right\rangle_{L^{2}} \\
& +\left\langle\gamma_{h_{j}}^{-2} \partial^{(2)} v_{j}, v_{j}\right\rangle_{L^{2}}+\left\langle\gamma_{h_{j}}^{-2} g^{\prime}\left(\Psi_{*}\right) v_{j}, v_{j}\right\rangle_{L^{2}} \\
& +\left\langle L_{c ; h_{j}}\left[v_{j}\right], v_{j}\right\rangle_{L^{2}} .
\end{aligned}
$$

Using $\left\langle v_{j}^{\prime}, v_{j}\right\rangle_{L^{2}}=0$, Lemma 6.12 and item (iii) of Proposition 6.1, we find

$$
\begin{aligned}
\left\langle y_{j}, v_{j}\right\rangle_{L^{2}} \leq & C_{2}^{\prime}\left[\left\|\partial^{+} v_{j}\right\|_{L_{\eta}^{2}}^{2}+\left\|v_{j}\right\|_{L_{\eta}^{2}}^{2}\right]+\left\langle\gamma_{h_{j}}^{-2} g^{\prime}\left(\Psi_{*}\right) v_{j}, v_{j}\right\rangle_{L^{2}} \\
\leq & C_{2}^{\prime}\left[\left\|\partial^{+} v_{j}\right\|_{L_{\eta}^{2}}^{2}+\left\|v_{j}\right\|_{L_{\eta}^{2}}^{2}\right] \\
& -\alpha\left\|v_{j}\right\|_{L^{2}}^{2}+C_{3}^{\prime} \int_{-m}^{m}\left|v_{j}(\tau)\right|^{2} d \tau .
\end{aligned}
$$

Using the basic inequality

$$
x y=(\sqrt{\alpha} x)(y / \sqrt{\alpha}) \leq \frac{\alpha}{2} x^{2}+\frac{1}{2 \alpha} y^{2},
$$

we arrive at

$$
\begin{aligned}
C_{3}^{\prime} \int_{-m}^{m}\left|v_{j}(\tau)\right|^{2} d \tau \geq & \alpha\left\|v_{j}\right\|_{L^{2}}^{2}-\left\langle y_{j}, v_{j}\right\rangle_{L^{2}} \\
& -C_{2}^{\prime}\left[\left\|\partial^{+} v_{j}\right\|_{L_{\eta}^{2}}^{2}+\left\|v_{j}\right\|_{L_{\eta}^{2}}^{2}\right] \\
\geq & \alpha\left\|v_{j}\right\|_{L^{2}}^{2}-\left\|y_{j}\right\|_{L^{2}}\left\|v_{j}\right\|_{L^{2}} \\
& -C_{2}^{\prime}\left[\left\|\partial^{+} v_{j}\right\|_{L_{\eta}^{2}}^{2}+\left\|v_{j}\right\|_{L_{\eta}^{2}}^{2}\right] \\
\geq & \frac{\alpha}{2}\left\|v_{j}\right\|_{L^{2}}^{2}-\frac{1}{2 \alpha}\left\|y_{j}\right\|_{L^{2}}^{2} \\
& -C_{2}^{\prime}\left[\left\|\partial^{+} v_{j}\right\|_{L_{\eta}^{2}}^{2}+\left\|v_{j}\right\|_{L_{\eta}^{2}}^{2}\right] .
\end{aligned}
$$

Multiplying the first inequality in (6.103) by $\frac{\alpha}{2\left(1+K_{2}\right)}$, we find

$$
0 \geq \frac{\alpha}{2\left(1+K_{2}\right)}\left\|v_{j}^{\prime}\right\|_{L^{2}}^{2}-\frac{\alpha K_{2}}{2\left(1+K_{2}\right)}\left\|v_{j}\right\|_{L^{2}}^{2}-C_{4}^{\prime}\left\|\partial^{+} v_{j}\right\|_{L_{\eta}^{2}}^{2}-C_{4}^{\prime}\left\|y_{j}\right\|_{L^{2}}^{2} .
$$

Adding (6.119) and (6.120), we may use the identity

$$
\frac{\alpha}{2}-\frac{\alpha K_{2}}{2\left(1+K_{2}\right)}=\frac{\alpha}{2\left(1+K_{2}\right)},
$$

to obtain

$$
\begin{aligned}
C_{3}^{\prime} \int_{-m}^{m}\left|v_{j}(\tau)\right|^{2} d \tau \geq & \frac{\alpha}{2\left(1+K_{2}\right)}\left[\left\|v_{j}\right\|_{L^{2}}^{2}+\left\|v_{j}^{\prime}\right\|_{L^{2}}^{2}\right]-C_{5}^{\prime}\left\|y_{j}\right\|_{L^{2}}^{2} \\
& -C_{5}^{\prime}\left[\left\|\partial^{+} v_{j}\right\|_{L_{\eta}^{2}}^{2}+\left\|v_{j}\right\|_{L_{\eta}^{2}}^{2}\right] \\
= & \frac{\alpha}{2\left(1+K_{2}\right)}-C_{5}^{\prime}\left\|y_{j}\right\|_{L^{2}}^{2} \\
& -C_{5}^{\prime}\left[\left\|\partial^{+} v_{j}\right\|_{L_{\eta}^{2}}^{2}+\left\|v_{j}\right\|_{L_{\eta}^{2}}^{2}\right] .
\end{aligned}
$$


For any $M \geq 0$ and $a \in L^{2}$ we may compute

$$
\begin{aligned}
\|a\|_{L_{\eta}^{2}}^{2} & =\int e^{-2 \eta|\tau|} a(\tau)^{2} d \tau \\
& \leq e^{-2 \eta M}\|a\|_{L^{2}}^{2}+\int_{-M}^{M} e^{-2 \eta|\tau|} a(\tau)^{2} d \tau \\
& \leq e^{-2 \eta M}\|a\|_{L^{2}}^{2}+\int_{-M}^{M} a(\tau)^{2} d \tau .
\end{aligned}
$$

Exploiting $\left\|\partial^{+} v_{j}\right\|_{L^{2}} \leq\left\|v_{j}^{\prime}\right\|_{L^{2}}$ and $\left\|v_{j}\right\|_{H^{1}}=1$, we hence see

$$
\left\|v_{j}\right\|_{L_{\eta}^{2}}+\left\|\partial^{+} v_{j}\right\|_{L_{\eta}^{2}}^{2} \leq e^{-2 \eta M}+\int_{-M}^{M}\left[v_{j}\right](\tau)^{2} d \tau+\int_{-M}^{M}\left[\partial^{+} v_{j}\right](\tau)^{2} d \tau .
$$

In particular, by choosing $M \geq m$ to be sufficiently large, we find

$$
\begin{aligned}
C_{3}^{\prime} \int_{-M}^{M}\left|v_{j}(\tau)\right|^{2} d \tau \geq & C_{3}^{\prime} \int_{-m}^{m}\left|v_{j}(\tau)\right|^{2} d \tau \\
\geq & \frac{\alpha}{4\left(1+K_{2}\right)}-C_{5}^{\prime}\left\|f_{j}\right\|_{L^{2}}^{2} \\
& -C_{5}^{\prime}\left[\int_{-M}^{M}\left[\partial^{+} v_{j}\right](\tau)^{2} d \tau+\int_{-M}^{M} v_{j}(\tau)^{2} d \tau\right] .
\end{aligned}
$$

We hence obtain

$$
C_{6}^{\prime}\left[\int_{-M}^{M}\left[\partial^{+} v_{j}\right](\tau)^{2} d \tau+\int_{-M}^{M} v_{j}(\tau)^{2} d \tau\right] \geq \frac{\alpha}{4\left(1+K_{2}\right)}-C_{5}^{\prime}\left\|y_{j}\right\|_{L^{2}}^{2} .
$$

In view of the bound

$$
\limsup _{j \rightarrow \infty}\left\|y_{j}\right\|_{L^{2}}^{2} \leq \mu(\delta)^{2}
$$

the strong convergences $v_{j} \rightarrow V_{*} \in L^{2}([-M, M])$ and $\partial^{+} v_{j} \rightarrow V_{*}^{\prime} \in L^{2}([-M, M])$ imply that

$$
\left\|V_{*}\right\|_{H^{1}}^{2} \geq\left[C_{6}^{\prime}\right]^{-1}\left[\frac{\alpha}{4\left(1+K_{2}\right)}-C_{5}^{\prime} \mu(\delta)^{2}\right],
$$

as desired. The bound for $W_{*}$ follows in a very similar fashion.

Proof of Proposition 6.6 For any $0<\delta<1$, Lemma's 6.9 and 6.13 show that the function $V_{*}$ defined in Lemma 6.8 satisfies

$$
K_{1}^{2} \mu(\delta)^{2} \geq\left\|V_{*}\right\|_{H^{1}}^{2} \geq K_{3}-K_{4} \mu(\delta)^{2},
$$

which gives $\left(K_{1}^{2}+K_{4}\right) \mu(\delta)^{2} \geq K_{3}>0$, as desired. The same computation works for $\mu^{\text {adj }}$, but now one uses Lemma's 6.10 and 6.13.

Acknowledgements Hupkes acknowledges support from the Netherlands Organization for Scientific Research (NWO) (Grant 639.032.612). Van Vleck acknowledges support from the NSF (DMS-1419047 and DMS1714195). Both authors wish to thank W. Huang for helpful discussions during the conception and writing of this paper.

Open Access This article is licensed under a Creative Commons Attribution 4.0 International License, which permits use, sharing, adaptation, distribution and reproduction in any medium or format, as long as you give appropriate credit to the original author(s) and the source, provide a link to the Creative Commons licence, and indicate if changes were made. The images or other third party material in this article are included in the article's Creative Commons licence, unless indicated otherwise in a credit line to the material. If material is not included in the article's Creative Commons licence and your intended use is not permitted by statutory regulation or exceeds the permitted use, you will need to obtain permission directly from the copyright holder. To view a copy of this licence, visit http://creativecommons.org/licenses/by/4.0/. 


\section{A Auxiliary results}

In this short appendix we collect several useful results from [4] that are used throughout this paper. In particular, we recall a number of basic identities related to discrete differentiation and integration in Sect. A.1. In addition, we formulate some useful bounds for the gridpoint spacing function $\gamma_{U}$ in Sect. A.2.

\section{A.1 Discrete calculus}

Recalling the notation introduced at the start of Sect. 5, a short computation yields the basic identities

$$
\partial^{(2)} a=\partial^{+} \partial^{-} a, \quad \partial^{+} \partial^{0} a=S^{+}\left[\partial^{(2)} a\right],
$$

together with the product rules

$$
\begin{aligned}
& \partial^{+}[a b]=\partial^{+} a T^{+} b+a \partial^{+} b, \\
& \partial^{0}[a b]=\partial^{0} a T^{+} b+T^{-} a \partial^{0} b, \\
& \partial^{-}[a b]=\left[\partial^{-} a\right] b+\left[T^{-} a\right] \partial^{-} b,
\end{aligned}
$$

which hold for $a, b \in \ell_{h}^{\infty}$. As in [4, Sect. 3.1], these can subsequently be used to derive the second-order product rule

$$
\partial^{(2)}[a b]=\left(\partial^{(2)} a\right) b+\partial^{+} a \partial^{+} b+\partial^{-} a \partial^{-} b+a \partial^{(2)} b .
$$

Recalling the discrete summation operators (2.25), one can read-off the identities

$$
\partial^{+}\left[\sum_{-; h} a\right]_{j h}=a_{j h}, \quad \partial^{-}\left[\sum_{+; h} a\right]_{j h}=-a_{j h}
$$

for $a \in \ell^{1}(h \mathbb{Z} ; \mathbb{R})$. In addition, the discrete summation-by-parts identity

$$
\sum_{-; h} b \partial^{+} a=a T^{-} b-\sum_{-; h} a \partial^{-} b
$$

holds whenever $a, b \in \ell_{h}^{2}$; see [4, Eq. (3.13)].

\section{A.2 Bounds for $\gamma_{U}$}

For any $U^{(a)}, U^{(b)} \in \Omega_{h ; \kappa}$, the gridspace function $\gamma_{U}$ defined in (5.1) admits the identity

$$
\gamma_{U^{(b)}}-\gamma_{U^{(a)}}=-\left[\gamma_{U^{(a)}}+\gamma_{U^{(b)}}\right]^{-1}\left(\partial^{0} U^{(a)}+\partial^{0} U^{(b)}\right)\left(\partial^{0} U^{(b)}-\partial^{0} U^{(a)}\right) ;
$$

see [4, Eq. (C.4)]. This can be used [4, Cor. D.2] to obtain the Lipschitz bound

$$
\left\|\gamma_{U^{(a)}}-\gamma_{U^{(b)}}\right\|_{\ell_{h}^{q}} \leq K\left\|\partial^{+} U^{(b)}-\partial^{+} U^{(a)}\right\|_{\ell_{h}^{q}}
$$

for $q \in\{2, \infty\}$, where $K$ depends on $\kappa$ but not on $h$. In addition, it can be exploited to compute the following bounds concerning discrete differences of powers of $\gamma_{U}$. 
Lemma A.1 ([4, Lem. D.4]) Fix $0<\kappa<\frac{1}{12}$. Then there exists $K>0$ so that for any $h>0$ and any $U \in \Omega_{h ; \kappa}$, we have the pointwise estimates

$$
\begin{aligned}
& \left|\partial^{+}\left[\gamma_{U}^{2}\right]+2 \partial^{0} U S^{+}\left[\partial^{(2)} U\right]\right| \leq K h\left[\left|\partial^{(2)} U\right|^{2}+T^{+}\left|\partial^{(2)} U\right|^{2}\right], \\
& \mid \partial^{+}\left[\gamma_{U}\right]+\gamma_{U}^{-1} \partial^{0} U S^{+}\left[\partial^{(2)} U\right] \leq K h\left[\left|\partial^{(2)} U\right|^{2}+T^{+}\left|\partial^{(2)} U\right|^{2}\right], \\
& \left|\partial^{+}\left[\gamma_{U}^{-1}\right]-\gamma_{U}^{-3} \partial^{0} U S^{+}\left[\partial^{(2)} U\right]\right| \leq K h\left[\left|\partial^{(2)} U\right|^{2}+T^{+}\left|\partial^{(2)} U\right|^{2}\right], \\
& \left|\partial^{+}\left[\gamma_{U}^{-2}\right]-2 \gamma_{U}^{-4} \partial^{0} U S^{+}\left[\partial^{(2)} U\right]\right| \leq K h\left[\left|\partial^{(2)} U\right|^{2}+T^{+}\left|\partial^{(2)} U\right|^{2}\right], \\
& \left|\partial^{+}\left[\gamma_{U}^{-4}\right]-4 \gamma_{U}^{-6} \partial^{0} U S^{+}\left[\partial^{(2)} U\right]\right| \leq K h\left[\left|\partial^{(2)} U\right|^{2}+T^{+}\left|\partial^{(2)} U\right|^{2}\right] .
\end{aligned}
$$

\section{References}

1. Bates, P.W., Chen, X., Chmaj, A.: Traveling waves of bistable dynamics on a lattice. SIAM J. Math. Anal. 35, 520-546 (2003)

2. Fife, P.C., McLeod, J.B.: The approach of solutions of nonlinear diffusion equations to travelling front solutions. Arch. Ration. Mech. Anal. 65(4), 335-361 (1977)

3. Haragus, M., Scheel, A.: Corner defects in almost planar interface propagation. Annales de l'Institut Henri Poincare (C) Non Linear Analysis, vol. 23, pp. 283-329. Elsevier, Amsterdam (2006)

4. Hupkes, H.J., Van Vleck, E.S.: Travelling waves for adaptive grid discretizations of reaction diffusion systems I: well-posedness. J. Dyn. Differ. Equ. 28, 955 (2016)

5. Hupkes, H.J., Van Vleck, E.S.: Travelling waves for adaptive grid discretizations of reaction diffusion systems III: nonlinear theory

6. Hupkes, H.J., Van Vleck, E.S.: Travelling waves for complete discretizations of reaction diffusion systems. J. Dyn. Diff. Eqn. 28(3), 955-1006 (2016)

7. Johann, A.: An application for an implicit function theorem of Craven and Nashed: continuum limits of lattice differential equations. J. Math. Anal. Appl. 367(2), 389-400 (2010)

8. Kapitula, T.: Multidimensional stability of planar travelling waves. Trans. Am. Math. Soc. 349, 257-269 (1997)

9. Kapitula, T., Promislow, K.: Spectral and Dynamical Stability of Nonlinear Waves. Springer, New York (2013)

10. Sattinger, D.: Weighted norms for the stability of traveling waves. J. Differ. Equ. 25(1), 130-144 (1977)

11. Schouten, W., Hupkes, H .J.: Nonlinear stability of pulse solutions for the discrete FitzHugh-Nagumo equation with Infinite-Range Interactions. Discrete Contin. Dyn. Syst. 39(9), 5017-5083 (2019). https:// doi.org/10.3934/dcds.2019205

Publisher's Note Springer Nature remains neutral with regard to jurisdictional claims in published maps and institutional affiliations. 